\title{
MICHAEL'S PROBLEM AND THE POINCARÉ-FATOU-BIEBERBACH PHENOMENON
}

\author{
P. G. DIXON AND J. ESTERLE
}

1. Introduction. Let $A$ be a Fréchet algebra, i.e. a complete, metrizable topological algebra whose topology is defined by an increasing family $\left(q_{n}\right)$ of submultiplicative seminorms. It is still not known whether characters on $A$ are necessarily continuous. This is the classical "problem of Michael" raised in 1952 by Michael in [73]. The starting point of this paper is a new approach to this problem. We show in Theorem 3.3 that if discontinuous characters do exist on some Fréchet algebras, then for every system $\left(\mathbf{C}^{p_{n}}, F_{n}\right)_{n \geqslant 1}$, where $\left(p_{n}\right)$ is a sequence of nonnegative integers and where $F_{n}: \mathbf{C}^{p_{n+1}} \rightarrow \mathbf{C}^{p_{n}}$ is entire for every $n \geqslant 1$, there exists a sequence $\left(z_{n}\right)$ in $\Pi_{n \geqslant 1} \mathbf{C}^{p_{n}}$ such that $z_{n}=F_{n}\left(z_{n+1}\right)$ $(n \geqslant 1)$. In particular, if it were possible to construct for some $p \geqslant 1$ a sequence $\left(F_{n}\right)$ of entire functions from $\mathbf{C}^{p}$ into itself such that $\bigcap_{n \geqslant 1}\left(F_{1} \circ \ldots \circ F_{n}\right)\left(\mathbf{C}^{p}\right)=\varnothing$ then the answer to Michael's problem would be positive, i.e., all characters on Fréchet algebras would be continuous. It follows of course from the big Picard theorem that no such sequence exists for $p=1$, but if $p \geqslant 2$ it is well known that there exists a one-to-one entire function $F: \mathbf{C}^{p} \rightarrow \mathbf{C}^{p}$, whose jacobian identically equals 1 but whose range is not dense in $\mathbf{C}^{p}$. Such a function was constructed by Bieberbach in [11] for $p=2$ (see also the very clear exposition of Bieberbach's construction presented by Stehlé in [101], where the argument is extended to all $p \geqslant 2$ ). Another example of a nondegenerate entire function from $\mathbf{C}^{2}$ into itself whose range is not dense was given previously by Fatou in [46] (but, despite some claims of the contrary, Fatou's function is not one-to-one, see §6). In fact this phenomenon was explicitly pointed out by Poincaré in $[85$, p. 333] forty years before Bieberbach. All these constructions rely on the theory of normal forms for local analytic automorphisms with a repulsive fixed point, and suggest that if $p \geqslant 2$ a sequence $\left(F_{n}\right)$ with $\bigcap_{n \geqslant 1}\left(F_{1} \circ \cdots \circ F_{n}\right)\left(\mathbf{C}^{p}\right)=\varnothing$ might exist. We were not able to decide whether this is true or not, but we give here a new method to construct these "Bieberbach functions". This leads to new examples of one-toone entire functions over $\mathbf{C}^{2}$, of jacobian 1 , whose range is not dense, given in $\S 8$. We use these functions to construct strange nondegenerate entire functions from $\mathbf{C}^{2}$ into itself.

Received by the editors August 29, 1985.

1980 Mathematics Subject Classification (1985 Revision). Primary 32H25; Secondary 46H05, 32-03, 01A55, 01A60. 
On the one hand, we show that for every $\varepsilon>0$ there exists a function $F$ over $\mathbf{C}^{2}$, whose jacobian never vanishes, whose range is contained in the union of the two polycylinders $\left\{\left(z_{1}, z_{2}\right) \in \mathbf{C}^{2}|| z_{1} \mid<\varepsilon\right\}$ and $\left\{\left(z_{1}, z_{2}\right) \in \mathbf{C}^{2}|| z_{2} \mid<\varepsilon\right\}$. Since of course it is possible to arrange that $F^{\prime}(0)=I$, this shows how much Bloch's theorem fails for functions of two complex variables. It is even possible to arrange that $\sup \left\{\min \left\{\left|z_{1}\right|,\left|z_{2}\right|\right\}:\left(z_{1}, z_{2}\right) \in F\left(\mathbf{C}^{2}\right),\left|\left(z_{1}, z_{2}\right)\right|=R\right\} \rightarrow 0$ as $R \rightarrow \infty$ and to arrange for the volume of the intersection of $F\left(\mathbf{C}^{2}\right)$ with one of these polycylinders to be finite. It is also possible to arrange the range of $F$ to avoid a neighborhood of the set $X=\left\{\left(z_{1}, z_{2}\right) \in \mathbf{C}^{2}: z_{1} \cdot z_{2}=0\right\}$.

In the other direction, we show that for every $\varepsilon>0$ there exists an entire function $G: \mathbf{C}^{2} \rightarrow \mathbf{C}^{2}$ such that $G\left(\mathbf{C}^{2}\right)$ does not intersect the polydisc of radius 1 centered at the origin but contains the complement of the polydisc of radius $1+\varepsilon$ centered at the origin. These examples seem to be quite new. We also point out that if $F$ is Bieberbach's original function (or one of Sadullaev's related functions [93]) then there exists a bounded subset $B$ of $\mathbf{C}^{2}$ such that $(F \circ H)\left(\mathbf{C}^{2}\right) \cap B \neq \varnothing$ for every nonconstant entire mapping $H: \mathbf{C}^{2} \rightarrow \mathrm{C}^{2}$ (a similar property holds for the function $F$ whose range is contained in the union of two small polycylinders described above). Of course if $F$ is one of the above functions, we have $(\Theta \circ F)\left(\mathbf{C}^{2}\right) \cap D(0,1)=\varnothing$ for some suitable affine automorphism $\Theta$ of $\mathbf{C}^{2}$. But if one tries to construct by induction a sequence $\left(F_{n}\right)$ of entire mappings from $\mathbf{C}^{2}$ into itself such that $\left(F_{1} \circ \ldots \circ F_{n}\right)\left(\mathbf{C}^{2}\right) \cap$ $D(0, n)=\varnothing(n \geqslant 1)$, to take $F_{1}=\Theta \circ F$ would be really a bad start.

In fact, if $G: \mathbf{C}^{p} \rightarrow \mathbf{C}^{p}$ is a one-to-one holomorphic mapping, and if $\alpha \in \operatorname{Fr}\left(G\left(\mathbf{C}^{p}\right)\right)$ then $G^{-1}(V)$ is unbounded for every neighborhood $V$ of $\alpha$. We were not able to get enough control on inverse images of bounded sets to obtain one-to-one functions $G: \mathbf{C}^{2} \rightarrow \mathbf{C}^{2}$ with nondense range such that for every bounded subset $B$ of $\mathbf{C}^{2}$ there exists a one-to-one (or even a nonconstant) holomorphic mapping $H_{B}: \mathbf{C}^{2} \rightarrow \mathbf{C}^{2}$ such that $H_{B}\left(\mathbf{C}^{2}\right) \cap G^{-1}(B)=\varnothing$. Some rather precise information is nevertheless obtained in $\S 8$ in the case of a function $G$ whose range avoids the set $\left\{\left(z_{1}, z_{2}\right) \in \mathbf{C}^{2} \mid \operatorname{Re} z_{1}>\varepsilon, \operatorname{Re} z_{2}>\varepsilon\right\}$. Outside a "critical zone" which is a union of thin vertical and horizontal tubes, $G^{-1}$ acts like real horizontal and vertical translations, whose magnitude is bounded on bounded sets, and some qualitative information on the inverse images of bounded sets is obtained.

We now give a brief outline of the paper. $\$ \S 2$ and 3 are the ones related to Michael's problem. Clayton and Schottenloher $[26,94]$ gave examples of commutative Fréchet algebras $A$ which are "universal" for this problem, in the sense that if there exist discontinuous characters on some commutative Fréchet algebra there must exist some on $A$. We give in $\$ 2$ a very simple construction of such an algebra $\mathscr{E}$, which is "universal" for the general case, in the sense that if there exists a discontinuous character on some Fréchet algebra, commutative or not, there must exist a discontinuous character on $\mathscr{E}$.

In $\S 3$ we establish the connection between Michael's problem and projective systems of entire functions of several complex variables described above. The proof uses the fact that if $f: \mathbf{C}^{p} \rightarrow \mathbf{C}$ is an entire function, then $\chi\left[f\left(a_{1}, \ldots, a_{p}\right)\right]=f\left[\chi\left(a_{1}\right), \ldots, \chi\left(a_{p}\right)\right]$ for every family $\left(a_{1}, \ldots, a_{p}\right)$ of elements of $A$ and for every character $\chi$, continuous or not, on $A$, and a trick 
based upon the Mittag-Leffler theorem on projective limits of complete metrizable spaces, a usual tool in recent developments in Banach algebra theory (see for example $[42,43,45,114])$. When $A$ is commutative, the fact that characters commute with entire functions is an immediate consequence of a fundamental result of Arens [8], which shows that characters are continuous on commutative Fréchet algebras polynomially (or rationally) generated by a finite number of elements. Note that this result of Arens, which is the only consistent general partial result about Michael's problem (using the Remmert-Narasimhan-Bishop embedding theorem, it is possible to deduce from Arens's theorem that the only characters on $\mathcal{O}(U)$, the algebra of holomorphic functions on a Stein manifold, are point evaluations) was based itself upon the Mittag-Leffler theorem.

The remainder of the paper is devoted to the study of the range of nondegenerate, or one-to-one, entire functions from $\mathbf{C}^{p}$ into itself. In $\S 4$ we observe that the convex hull of the range of such a function must equal $\mathbf{C}^{p}$, and we collect some information. For example, the range of $F$ cannot avoid more than $p$ complex hyperplanes (Green [50]). This follows from BorelNevanlinna theory [14,75] (see also Ahlfors [2]). Also, if $F$ is one-to-one, $\mathbf{C}^{p} \backslash F\left(\mathbf{C}^{p}\right)$ has to be unbounded and the convex hull of a connected tube contained in $F\left(\mathbf{C}^{p}\right)$ cannot equal $\mathbf{C}^{p}$ unless $F$ is onto (this follows from Hartogs' theorem and from a related extension theorem for tubes). We also mention an interesting result of Sibony and Pit-Mann Wong [98] which shows that if an entire function $F: \mathbf{C}^{p} \rightarrow \mathbf{C}^{p}$ grows sufficiently slowly then the range of $F$ is dense (it is also shown in [98] that there exists for every $\varepsilon>0$ one-to-one entire functions over $\mathbf{C}^{p}(p \geqslant 2)$ of jacobian 1 , which are of exponential type $\varepsilon$ but have nondense range).

In $\S 5$ we investigate sequences $\left(\Theta_{n}\right)$ of analytic automorphisms on $\mathbf{C}^{p}$ which converge uniformly on compact sets to an entire function $F$. If $F$ is nondegenerate (which is indeed the case if the jacobian of $\Theta_{n}$ is identically equal to 1 for each $n$ ) then $F$ is one-to-one, $F\left(\mathbf{C}^{p}\right)$ is a Runge domain, $\Theta_{n}^{-1}(z)$ converges to $F^{-1}(z)$ uniformly on compact subsets of $F\left(\mathbf{C}^{p}\right)$ and $\left|\Theta_{n}^{-1}(z)\right|_{n \rightarrow \infty} \infty$ uniformly on $\mathbf{C}^{p} \backslash F\left(\mathbf{C}^{p}\right)$. These observations are related to well-known (and well-forgotten) results of $\mathrm{H}$. Cartan [23, Lemme 5]. H. Cartan used Kronecker integrals, but it is possible to deduce these facts from Cauchy's inequalities and from the fixed point theorem [44]. Related results show that if a sequence $\left(F_{n}\right)$ of entire functions from $\mathbf{C}^{p}$ into itself converges to the identity map uniformly on compact sets then every compact subset of $\mathbf{C}^{p}$ is eventually contained in $F_{n}\left(\mathbf{C}^{p}\right)$. This contradicts a minor statement in [98, Proposition 1.1].

In $\$ 6$ we describe the classical method of constructing an entire function from $\mathbf{C}^{p}$ into itself whose range is not dense. If $\Theta$ is the germ of a local automorphism with repulsive fixed point at the origin, then it follows from the theory of "normal forms" that there exists a polynomial automorphism $B$ of $\mathbf{C}^{p}$, with $B^{-n}(z) \underset{n \rightarrow \infty}{\rightarrow} 0$ for every $z \in \mathbf{C}^{p}$, and a germ of a local automorphism $F$ at the origin satisfying $\Theta \circ F=F \circ B$. If there exists an open subset $U$ of $\mathbf{C}^{p}$ containing the origin such that $\Theta$ is defined and analytic over $U$ and such that $\Theta(U) \subset U$, then $F$ extends to an entire function, and $F\left(\mathbf{C}^{p}\right)$ is the set of all 
$z \in U$ such that $z=\Theta^{n}\left(z_{n}\right)(n \geqslant 1)$ for some sequence $z_{n}$ satisfying $z_{n} \rightarrow_{n \rightarrow \infty} 0$. If $\Theta$ is an analytic automorphism of $\mathbf{C}^{p}$ then $F\left(\mathbf{C}^{p}\right)=\{z \in$ $\left.\mathrm{C}^{p} \mid \Theta^{-n}(z) \underset{n \rightarrow \infty}{\rightarrow} 0\right\}$, and $F$ is one-to-one. So if $\Theta$ has another repulsive fixed point, $F\left(\mathbf{C}^{p}\right)$ is not dense in $\mathbf{C}^{p}$. This observation was made explicitly by Poincaré in 1890 [85, p. 333] in a paper where the functional equation $\Theta \circ F=F \circ \Theta^{\prime}(0)$ was solved in the case where $\Theta$ is a birational map satisfying $\Theta(0)=0, \Theta^{\prime}(0)=k I$ with $|k|>1$; more general functional equations were studied by Picard [82, 83] but, despite a claim of Picard in his 1904 paper [83], it is not possible in general to solve the functional equation $\Theta \circ F=F \circ \Theta^{\prime}(0)$ if 0 is a repulsive fixed point for $\Theta$, because some "relations" of the form $\lambda_{1}=\lambda_{1}^{m_{1}} \cdots \lambda_{p}^{m_{p}}$ with $m_{1} \geqslant 0, \ldots, m_{p} \geqslant 0, m_{1}+\cdots+m_{p} \geqslant 2$ may occur between the eigenvalues $\lambda_{1}, \ldots, \lambda_{p}$ of $F^{\prime}(0)$ at 0 . A general precise form for the "normal form" $B$ of $\Theta$ was given by Reich in $1969[87,88]$, but Reich's work overlaps a previous work of Sternberg [102], and also overlaps the 1897 thesis of Leau [67], where the formal aspect is treated in full detail and where the analytic aspect is treated with a technical extra hypothesis. If $p=1$, no "relations" may occur with a repulsive fixed point, and the corresponding functional equation, called Schröder equation, was entirely solved in 1882 by Koenigs [62]. We also point out in $\$ 6$ the connection between the Jordan-Chevalley decomposition theorem in algebraic groups and the formal aspect of the theory.

The theory of normal forms for local invertible mappings has a well-known connection with the theory of normal forms for some differential equations which present a singularity at the origin. In this topic the seminal paper is Poincarés thesis [84], and the difficulty caused by "resonances", the counterpart of the "relations" mentioned above, was circumvented by Dulac [37]. References to recent works about local invertible mappings with fixed points which are neither attractive nor repulsive, and about the corresponding case for differential equations and holomorphic flows, are given in $\$ 6$ (see in particular the talks given by Malgrange [68] and Martinet [70] at the Bourbaki seminar, and Siegel's basic paper [99]).

The above considerations about functional equations lead to a "dynamical" characterization of open subsets $U$ of $\mathbf{C}^{p}$ which contain the range of a nondegenerate entire mapping $G: \mathbf{C}^{p} \rightarrow \mathbf{C}^{p}$. A necessary and sufficient condition is the existence of an analytic mapping $\Theta: U \rightarrow U$ which possesses a repulsive fixed point in $U$. In order for $U$ to be biholomorphically equivalent to $\mathbf{C}^{p}$ it is necessary and sufficient that there exists an analytic automorphism $\Theta$ of $U$ such that $\Theta^{-n}(z) \underset{n \rightarrow \infty}{\rightarrow} \alpha$ for every $z \in U$, where $\alpha$ is some (repulsive) fixed point of $\Theta$ in $U$ (the latter statement is a special case of a theorem of Kato [60]). We give some simple ways to construct examples of infinite sequences of one-to-one entire functions on $\mathbf{C}^{2}$ with disjoint ranges, including an unpublished example of W. Rudin (the first such example is due to Kodaira [61]). Also we present an unpublished very short argument of W. Rudin which gives a method of solving the functional equation $F \circ G=G \circ F^{\prime}(0)$ in a special case which suffices for most applications. Some elementary computations giving information about the range of Bieberbach's original example and 
Sadullaev's related functions [93] are presented and we show that Fatou's original example is not one-to-one.

The range of Bieberbach's original function is the set

$$
\left\{z \in \mathbf{C}^{p} \mid \Theta^{-n}(z) \underset{n \rightarrow \infty}{\rightarrow} 0\right\},
$$

where $\Theta$ is a polynomial automorphism, and the range of Fatou's function is the set $\left\{z \in \mathbf{C}^{p} \mid \Psi^{n}(z) \underset{n \rightarrow \infty}{\rightarrow} 0\right\}$, where $\Psi$ is a rational map. After the work of Julia [58] before 1920, the study of iteration of polynomial and rational maps has recently undergone a renewal of interest, especially in dimension 1 , see the talk by Douady [35] at the Bourbaki seminar for references. The boundary of sets of the above forms is in general very complicated.

In $\$ 7$ we establish a (folklore) result which shows that for every sequence $\left(F_{n}\right)$ of nondegenerate entire functions from $\mathbf{C}^{p}$ into itself there exists an entire function $G$ with $\left(G \circ F_{1} \circ \cdots \circ F_{n}\right)\left(\mathbf{C}^{p}\right)=\mathbf{C}^{p}(n \geqslant 1)$. This result does not give any information about the sequence $\left(\left(F_{1} \circ \cdots \circ F_{n}\right)\left(\mathbf{C}^{p}\right)\right)_{n \geqslant 1}$, since $G$ is not one-to-one, but we obtain as a corollary the fact, due to Gauthier-Ngô Van Quê [49], that onto functions are dense in $\mathscr{H}\left(\mathbf{C}^{p}, \mathbf{C}^{p}\right)$. We show also that there exists a sequence $\left(F_{n}\right)$ of "Bieberbach functions" (i.e., entire mappings $\mathbf{C}^{p} \rightarrow$ $\mathbf{C}^{p}$ which are uniform limits on compact sets of analytic automorphisms of jacobian 1) such that $\bigcap_{n \geqslant 1}\left(F_{1} \circ \ldots \circ F_{n}\right)\left(\mathbf{C}^{p}\right)$ has empty interior and such that $\operatorname{Fr}\left(F_{1}\left(\mathbf{C}^{p}\right)\right) \subset \operatorname{Fr}\left(\left(F_{1} \circ F_{2}\right)\left(\mathbf{C}^{p}\right)\right) \subset \cdots \subset \operatorname{Fr}\left(\left(F_{1} \circ \cdots \circ F_{n}\right)\left(\mathbf{C}^{p}\right)\right) \cdots$. We also deduce from the computations of $\S 6$ the unpleasant properties of Bieberbach's original example (and of Sadullaev's examples) mentioned at the beginning of the introduction, which imply that there exist Bieberbach functions $G$ such that $\bigcap_{n \geqslant 1} \overline{\left(G \circ F_{1} \circ \cdots \circ F_{n}\right)\left(\mathbf{C}^{2}\right)} \neq \varnothing$ for every sequence $\left(F_{n}\right)$ of entire mappings $\mathbf{C}^{2} \rightarrow \mathbf{C}^{2}$.

In $\S 8$ we use an idea suggested by the results of $\S 5$ to obtain a new method of constructing "Bieberbach functions". The idea consists in producing a sequence $\left(\Theta_{n}\right)$ of analytic automorphisms of $\mathbf{C}^{2}$ of jacobian 1 which converges uniformly on compact sets to a function $F$, but which is such that $\left|\Theta_{n}^{-1}(z)\right|_{n \rightarrow \infty}^{\rightarrow} \infty$ for every element $z$ of a suitable set $\Delta$. Then $\Delta \cap F\left(\mathbf{C}^{2}\right)=\varnothing$ (Bieberbach's original example, Sadullaev's examples and Kodaira's example can be obtained this way, see §6). The key to obtaining the sequence of automorphisms we need is given by Arakelian's theorem [6] about uniform approximation by entire functions on some unbounded closed subsets $V$ of $\mathbf{C}$ (which extends Roth's theorem [90]).

This leads to the construction of the functions described above, and we outline at the end of the section an "Arakelian game" which can be played to construct other Bieberbach functions along the same lines.

Another interest in this method is that it points out the fact that these Bieberbach functions are not an isolated pathological phenomenon. The phenomenon is that the group of analytic automorphisms of jacobian 1 is not closed in $\mathscr{H}\left(\mathbf{C}^{p}, \mathbf{C}^{p}\right)$ when $p \geqslant 2$. This point of view is to be related with the theory of "Cohen elements" in separable commutative Banach algebras $A$ with bounded approximate identities $[42,86,114]$. These elements, which of course 
are not invertible, are limits in a strong sense of invertible elements of $A \oplus \mathbf{C} e$. They form a set which was rich enough to give a key for the second author's construction of discontinuous homomorphisms from $\mathscr{C}(K)$ [42] (about discontinuous homomorphisms from $\mathscr{C}(K)$ see also Dales' construction [29]).

In other terms, Bieberbach functions come from the fact that there exist analytic automorphisms of $\mathbf{C}^{p}$ which are close to the identity on large compact sets but perform very large deformations somewhere. A similar phenomenon was used by Skoda [100] to construct a counterexample to a long-standing conjecture of Serre.

The idea of a connection between Bieberbach functions and the continuity of characters on topological algebras first arose in [32], where the first author constructed a complete locally convex algebra with unbounded characters. This construction used, crucially, the fact that an analogue of Picard's theorem holds for polynomial mappings $\mathbf{C}^{p} \rightarrow \mathbf{C}^{p}$. The existence of the PoincaréFatou-Bieberbach phenomenon is the most obvious obstacle to the extension of this technique to L.M.C. algebras (the existence of unbounded characters on complete L.M.C. algebras is equivalent to the existence of discontinuous characters on Fréchet algebras; see Dixon and Fremlin [33] and Akkar [3]). An intermediate problem is the existence of discontinuous characters on complete metrizable locally convex algebras. Here the fact that mappings $\mathbf{C}^{p} \rightarrow \mathbf{C}^{p}$ with slow growth must have dense range (Sibony and Wong [98]) might remove one of the obstacles to constructing a discontinuous character.

There was also a known indirect relation between Bieberbach functions and Arakelian's theorem. It follows from the Remmert-Narasimhan-Bishop embedding theorem for Stein manifolds that there exist proper embeddings from the open unit disc into $\mathbf{C}^{3}$, but the question of the existence of such a proper embedding into $\mathbf{C}^{2}$, raised by Hitotomatu [55], remained open for a while. It was solved by Nishino [78], who used the fact that the range of the original example of Bieberbach is a Runge domain (readers who are not fluent in Japanese are referred to Stehlé's paper [101]). But Arakelian's theorem is a known useful tool to obtain embedding theorems (see [4,64] for proper embeddings of punctured discs and annuli into $\mathbf{C}^{2}$ ).

The authors, who are not experts in the theory of analytic functions of several complex variables, hope that this paper will help to attract the attention of specialists in this field to the Poincaré-Fatou-Bieberbach phenomenon. The obstructions to the construction of a sequence $\left(F_{n}\right)$ of analytic mappings $\mathbf{C}^{p} \rightarrow \mathbf{C}^{p}$ such that $\bigcap_{n \geqslant 1}\left(F_{1} \circ \cdots \circ F_{n}\right)\left(\mathbf{C}^{p}\right)=\varnothing$ are related to problems of polynomially convex hulls. Also, probabilistic methods might be useful to disprove, if possible, the existence of such a sequence (see the proofs of the little and big Picard theorems based upon Brownian motion, $[\mathbf{3 0}, 31]$ ), and it would be interesting to relate this problem and the partial results obtained here to recent results in value distribution theory $[\mathbf{5 1}, \mathbf{5 2}, \mathbf{5 3}]$.

The authors wish to thank E. Amar, H. G. Dales, R. Gay, C. O. Kiselman, W. Rudin, H. Skoda, J. Wermer, W. Zame and many others for valuable discussions and we wish to thank W. Rudin, who kindly allowed us to use his unpublished work [91] here (see §6). We have also to give warm thanks to P. Gauthier, who suggested the use of Arakelian's theorem after hearing in 
Montreal an elementary weak version of some of the results of $\$ 8$ [44], and pointed out many interesting references.

Last, but not least, we wish to thank Mrs. Clairand, who does not speak English, for her careful typing of the manuscript.

2. Test algebras. Recall that a Fréchet algebra is a metrizable complete algebra whose topology is defined by an increasing family $\left(q_{m}\right)_{m \geqslant 1}$ of submultiplicative seminorms (this means that $q_{m}(x y) \leqslant q_{m}(x) q_{m}(y)$ for $x, y \in A$, $m \geqslant 1)$. We denote by $\Sigma$ the algebra of all formal power series in infinitely many noncommuting variables $X_{1}, \ldots, X_{n}, \ldots$ with complex coefficients, equipped with the formal product of series. Each element $f$ of $\Sigma$ can be written in the form $f=\sum_{(i) \in \Lambda} \lambda_{(i)} X^{(i)}$, where $\Lambda$ is the set of all finite subsets $(i)=\left(i_{1}, \ldots, i_{k}\right)$ of $\mathbf{N}$. Here $X^{(i)}=X_{i_{1}} \cdots X_{i_{k}}$ and $|(i)|=k$ for each element $(i)=\left(i_{1}, \ldots, i_{k}\right)$ of $\Lambda$ (we denote the empty set by $(0)$ and set $|(0)|=0$ so that $e=X^{(0)}$ is the unit element of $\left.\Sigma\right)$.

Set $\mathscr{E}=\left\{f=\sum_{(i) \in \Lambda} \lambda_{(i)} X^{(i)} \in \Sigma\left|p_{m}(f)=\sum_{(i) \in \Lambda}\right| \lambda_{(i)} \mid m^{|(i)|}<+\infty\right.$ for each $m \geqslant 1\}$. Then $\mathscr{E}$ is a Fréchet algebra with respect to the (increasing) sequence $\left(p_{m}\right)_{m \geqslant 1}$ of submultiplicative seminorms.

Proposition 2.1. If there exists a discontinuous character on some Fréchet algebra, then there exists a discontinuous character on $\mathscr{E}$.

Proof. A standard elementary result on Fréchet linear spaces shows that a linear form $f$ on a Fréchet linear space $\left(E,\left(q_{m}\right)_{m \geqslant 1}\right)$ is continuous if and only if $\sup _{n \geqslant 1}\left|f\left(a_{n}\right)\right|<+\infty$ for each bounded sequence $\left(a_{n}\right)$ of elements of $E$ (i.e., for each sequence $\left(a_{n}\right)$ of elements of $E$ such that $\sup _{n \geqslant 1} q_{m}\left(a_{n}\right)<+\infty$ for each $m \geqslant 1$ ).

If there exists a discontinuous character $\chi$ on a Fréchet algebra $A$ then there exists a bounded sequence $\left(a_{n}\right)_{n \geqslant 1}$ of elements of $A$ such that $\liminf _{n \rightarrow \infty}\left|\chi\left(a_{n}\right)\right|=+\infty$. We may assume that $A$ is unital (if not $\chi$ can be extended to $A \oplus \mathrm{C} e$ ). Consider the map

$$
\begin{aligned}
\varphi: \mathscr{E} & \rightarrow A \\
\sum_{(i) \in \Lambda} \lambda_{(i)} X^{(i)} & \rightarrow \sum_{(i) \in \Lambda} \lambda_{(i)} a^{(i)}
\end{aligned}
$$

where $a^{(0)}=e, a^{(i)}=a_{i_{1}} \cdots a_{i_{k}}$ if $(i)=\left(i_{1}, \ldots, i_{k}\right)$. The growth condition on the coefficients $\lambda_{(i)}$ ensures that the above map is well defined, since the sequence $\left(a_{n}\right)_{n \geqslant 1}$ is bounded and $\varphi$ is clearly a continuous algebra homomorphism from $\mathscr{E}$ into $A$.

Set $\Psi=\chi \circ \varphi$. Then

$$
\liminf _{n \rightarrow \infty}\left|\Psi\left(X_{n}\right)\right|=\liminf _{n \rightarrow \infty}\left|\chi\left(a_{n}\right)\right|=+\infty .
$$

Since $p_{m}\left(X_{n}\right)=m(m \geqslant 1, n \geqslant 1)$, the sequence $\left(X_{n}\right)_{n \geqslant 1}$ is bounded in $\mathscr{E}$ and $\Psi$ is discontinuous on $\mathscr{E}$.

REMARK 2.2. Denote by $\Delta$ and $\Omega$ the commutative analogues of $\Sigma$ and $\mathscr{E}$. Using a similar argument, we see that if there exists a discontinuous character on any commutative Fréchet algebra $A$, then there exists a discontinuous character on the commutative Fréchet algebra $\Omega$. The map $\chi \rightarrow\left(\chi\left(X_{n}\right)\right)_{n \geqslant 1}$ 
defines a bijection between the set of all continuous characters of $\mathscr{E}$ (or of $\Omega$ ) and the set $l^{\infty}$ of all bounded sequences of complex numbers. We will not go here into further study of the set of continuous characters of these algebras.

3. Michael's problem and entire functions of several complex variables. In the sequel we denote by $\mathscr{H}\left(U, \mathbf{C}^{q}\right)$ the set of all holomorphic functions $f: U \rightarrow \mathbf{C}^{q}$, where $U$ is an open subset of $\mathbf{C}^{p}$. Each element $f$ of $\mathscr{H}\left(U, \mathbf{C}^{q}\right)$ can of course be written in the form $f=\left(f_{1}, \ldots, f_{q}\right)$, where $f_{i}: U \rightarrow \mathbf{C}$ is holomorphic for each $i \leqslant q$.

Each $g \in \mathscr{H}\left(\mathbf{C}^{p}, \mathbf{C}\right)$ has a power series expansion

$$
g\left(z_{1}, \ldots, z_{p}\right)=\sum_{i_{1} \geqslant 0, \ldots, i_{p} \geqslant 0} \alpha_{i_{1}, \ldots, i_{p}} z_{1}^{i_{1}} \cdots z_{p}^{i_{p}}
$$

where the coefficients $\alpha_{i_{1}, \ldots, i_{p}}$ are complex numbers and where

$$
\sum_{i_{1} \geqslant 0, \ldots, i_{p} \geqslant 0}\left|\alpha_{i_{1}, \ldots, i_{p}}\right| R^{i_{1}+\cdots+i_{p}}<+\infty
$$

for each $R>0$. So the series

$$
\sum_{i_{1} \geqslant 0, \ldots, i_{p} \geqslant 0} \alpha_{i_{1}, \ldots, i_{p}} a_{1}^{i_{1}} \cdots a_{p}^{i_{p}}
$$

is convergent in $A$ for every family $\left(a_{1}, \ldots, a_{p}\right)$ of $p$ elements of a unital Fréchet algebra $A$. We denote the sum of this series by $g\left(a_{1}, \ldots, a_{p}\right)$. If $f=\left(f_{1}, \ldots, f_{q}\right) \in \mathscr{H}\left(\mathbf{C}^{p}, \mathbf{C}^{q}\right)$, we set

$$
f\left(a_{1}, \ldots, a_{p}\right)=\left(f_{1}\left(a_{1}, \ldots, a_{p}\right), \ldots, f_{q}\left(a_{1}, \ldots, a_{p}\right)\right) .
$$

The map $\left(a_{1}, \ldots, a_{p}\right) \rightarrow f\left(a_{1}, \ldots, a_{p}\right)$ is clearly a continuous map from $A^{p}$ into $A^{q}$ for each $f \in \mathscr{H}\left(\mathbf{C}^{p}, \mathbf{C}^{q}\right)$. If the unital Fréchet algebra $A$ is commutative a theorem of Arens [8, Theorem 7.1] shows that the restriction of any character of $A$ to the closed unital subalgebra $B$ of $A$ generated by $\left(a_{1}, \ldots, a_{p}\right)$ is continuous, so that $\chi\left(f\left(a_{1}, \ldots, a_{p}\right)\right)=f\left(\chi\left(a_{1}\right), \ldots, \chi\left(a_{p}\right)\right)$ for each $f \in$ $\mathscr{H}\left(\mathbf{C}^{p}, \mathbf{C}\right)$, for each family $\left(a_{1}, \ldots, a_{p}\right)$ of $p$ elements of $A$ and for each character, continuous or not, on $A$.

To deal with the noncommutative case we need to introduce some algebras of noncommutative formal power series.

Fix $p \geqslant 1$ and denote by $\Lambda_{p}$ the set of all finite families $\left(i_{1}, \ldots, i_{k}\right)$ of elements of the set $\{1, \ldots, p\}$, including the empty family denoted by (0). Set $|(0)|=0,\left|\left(i_{1}, \ldots, i_{k}\right)\right|=k$ and consider the algebra $\Sigma_{p}$ of all formal power series in $p$ noncommuting variables $X_{1}, \ldots, X_{p}$ with complex coefficients equipped with the formal product of series. Each $f \in \Sigma_{p}$ can be written in the form $f=\sum_{(i) \in \Lambda_{p}} \lambda_{(i)} X^{(i)}$.

Here $X^{(i)}=X_{i_{1}} \cdots X_{i_{k}}$ for each element $(i)=\left(i_{1}, \ldots, i_{k}\right)$ of $\Lambda_{p}$ and the coefficients $\lambda_{(i)}$ are complex numbers. Set

$$
\begin{aligned}
\mathscr{E}_{p}=\left\{f=\sum_{(i) \in \Lambda_{p}} \lambda_{(i)} X^{(i)} \in \Sigma_{p} \mid\right. & \\
p_{m}(f) & \left.=\sum_{(i) \in \Lambda_{p}}\left|\lambda_{(i)}\right| m^{|(i)|}<+\infty \text { for each } m \geqslant 1\right\} .
\end{aligned}
$$


Then $\mathscr{E}_{p}$ is a unital Fréchet algebra with respect to the increasing sequence $\left(p_{m}\right)_{m \geqslant 1}$ of submultiplicative seminorms. Note that $\mathscr{E}_{p}$ is a closed subalgebra of the Fréchet algebra $\mathscr{E}$ introduced in the previous section.

Proposition 3.1. All characters $\chi$ on the Fréchet algebra $\mathscr{E}_{p}$ are continuous and defined for every element $f=\sum_{(i) \in \Lambda_{p}} \lambda_{(i)} X^{(i)}$ of $\mathscr{E}_{p}$ by the formula

$$
\chi(f)=\sum_{(i) \in \Lambda_{p}} \lambda_{(i)} \dot{\Delta}^{(i)}
$$

where $\Delta^{(i)}=\chi\left(X_{i_{1}}\right) \cdots \chi\left(X_{i_{k}}\right)$ for each $(i)=\left(i_{1}, \ldots, i_{k}\right) \in \Lambda_{p}\left(\right.$ with $\left.\Delta^{(0)}=1\right)$.

Proof. Consider a character $\chi$ on $\mathscr{E}_{p}$. The series $\sum_{(i) \in \Lambda_{p}} \lambda_{(i)} \Delta^{(i)}$ converges in $\mathbf{C}$ for each element $f=\sum_{(i) \in \Lambda_{p}} \lambda_{(i)} X^{(i)}$ of $\mathscr{E}_{p}$. Denote its sum by $\varphi(f)$. The map $\varphi: f \rightarrow \varphi(f)$ is clearly a continuous character on $\mathscr{E}_{p}$.

A routine induction over $k$ shows that for each monomial $X^{(i)}=X_{i_{1}} \cdots X_{i_{k}}$ we have

$$
X^{(i)}=\left(X_{i_{1}}-\chi\left(X_{i_{1}}\right) e\right) Y_{(i), 1}+\cdots+\left(X_{i_{k}}-\chi\left(X_{i_{k}}\right) e\right) Y_{(i), k}+\varphi\left(X^{(i)}\right) e,
$$

where $Y_{(i), j} \in \mathscr{E}_{p}$ and

$$
p_{m}\left(Y_{(i), j}\right) \leqslant\left|\chi\left(X_{i_{1}}\right) \cdots \chi\left(X_{i_{j-1}}\right)\right| m^{k-j} \quad(1 \leqslant j \leqslant k, m \geqslant 1) .
$$

Set $\rho=1+\max _{1 \leqslant i \leqslant p}\left|\chi\left(X_{\mathrm{i}}\right)\right|$. We obtain

$$
\sum_{j=1}^{k} p_{m}\left(Y_{(i), j}\right) \leqslant m^{k} \text { if } m \geqslant \rho .
$$

Now consider any element $f=\sum_{(i) \in \Lambda_{p}} \lambda_{(i)} X^{(i)}$ of $\mathscr{E}_{p}$. For every $(i) \in \Lambda_{p}$ and every $m \geqslant \rho$ we have

$$
X^{(i)}=\left(X_{1}-\chi\left(X_{1}\right) e\right) Z_{(i), 1}+\cdots+\left(X_{p}-\chi\left(X_{p}\right) e\right) Z_{(i), p}+\varphi\left(X^{(i)}\right) e,
$$

where $Z_{(i), j} \in \mathscr{E}_{p}, p_{m}\left(Z_{(i), j}\right) \leqslant m^{|(i)|}(1 \leqslant j \leqslant p)$. So the series $\sum_{(i) \in \Lambda_{p}} \lambda_{(i)} Z_{(i), j}$ converges to an element $f_{j}$ of $\mathscr{E}_{p}$ for $j=1, \ldots, p$ and we obtain

$$
f=\sum_{j=1}^{p}\left(X_{j}-\chi\left(X_{j}\right) e\right) f_{j}+\varphi(f) e .
$$

So $\chi(f)=\varphi(f)$ for each $f \in \mathscr{E}_{p}$, hence $\chi=\varphi$ and the proposition is proved.

Corollary 3.2. Let $A$ be a unital Fréchet algebra. Then $\chi\left[f\left(a_{1}, \ldots, a_{p}\right)\right]=$ $f\left[\chi\left(a_{1}\right), \ldots, \chi\left(a_{p}\right)\right]$ for each $f \in \mathscr{H}\left(\mathbf{C}^{p}, \mathbf{C}\right)$, for each finite family $\left(a_{1}, \ldots, a_{p}\right)$ of $p$ elements of $A$ and for each character $\chi$, continuous or not, on $A$.

Proof. Let

$$
f\left(z_{1}, \ldots, z_{p}\right)=\sum_{i_{1} \geqslant 0, \ldots, i_{p} \geqslant 0} \alpha_{i_{1}, \ldots, i_{p}} z_{1}^{i_{1}} \cdots z_{p}^{i_{p}}
$$

be the power series expansion of $f$. Set

$$
\tilde{f}=\sum_{i_{1} \geqslant 0, \ldots, i_{p} \geqslant 0} \alpha_{i_{1}, \ldots, i_{p}} X_{1}^{i_{1}} \cdots X_{p}^{i_{p}} .
$$

Then $\tilde{f} \in \mathscr{E}_{p}$. Fix $a_{1}, \ldots, a_{p} \in A$ and for each $(i)=\left(i_{1}, \ldots, i_{k}\right) \in \Lambda_{p}$ set $a^{(i)}=a_{i_{1}} \cdots a_{i_{k^{*}}}$. The map $\Psi: \sum_{(i) \in \Lambda_{p}} \lambda_{(i)} X^{(i)} \rightarrow \sum_{(i) \in \Lambda_{p}} \lambda_{(i)} a^{(i)}$ is a continuous algebra homomorphism from $\mathscr{E}_{p}$ into $A$. It follows from the proposition that $(\chi \circ \Psi)\left(\sum_{(i) \in \Lambda_{p}} \lambda_{(i)} X^{(i)}\right)=\sum_{(i) \in \Lambda_{p}} \lambda_{(i)} \Delta^{(i)}$, where $\Delta^{(i)}=$ $(\chi \circ \Psi)\left(X_{i_{1}}\right) \cdots(\chi \circ \Psi)\left(X_{i_{k}}\right)$ for each element $(i)=\left(i_{1}, \ldots, i_{k}\right)$ of $\Lambda_{p}$. 
We obtain

$$
\begin{aligned}
\chi\left[f\left(a_{1}, \ldots, a_{p}\right)\right] & =\chi[\Psi(\tilde{f})] \\
& =\sum_{i_{1} \geqslant 0, \ldots, i_{p} \geqslant 0} \alpha_{i_{1}, \ldots, i_{p}} \chi\left(a_{1}\right)^{i_{1}} \cdots \chi\left(a_{p}\right)^{i_{p}} \\
& =f\left[\chi\left(a_{1}\right), \ldots, \chi\left(a_{p}\right)\right]
\end{aligned}
$$

and the corollary is proved.

Let $\left(E_{n}\right)$ be a countable family of complete metrizable topological spaces. Assume that for every $n \geqslant 1$ a map $\Theta_{n}: E_{n+1} \rightarrow E_{n}$ is given. The set $\lim \operatorname{proj}\left(E_{n}, \Theta_{n}\right)$ is the set of all elements $\left(x_{n}\right)_{n \geqslant 1}$ of the cartesian product $\prod_{n \geqslant 1} E_{n}$ satisfying $x_{n}=\Theta_{n}\left(x_{n+1}\right)(n \geqslant 1)$. Denote by $\pi_{1}$ the first coordinate projection $\left(x_{n}\right)_{n \geqslant 1} \rightarrow x_{1}$ on $\prod_{n \geqslant 1} E_{n}$. The classical Mittag-Leffler theorem shows that if $\Theta_{n}$ is continuous and if $\Theta_{n}\left(E_{n+1}\right)$ is dense in $E_{n}$ for every $n \geqslant 1$, then $\pi_{1}\left(\lim \operatorname{proj}\left(E_{n}, \Theta_{n}\right)\right)$ is dense in $E_{1}$. See for example Arens [8, Theorem 2.4] or [43, Theorem 2.14] for a proof. People interested in the French way of making obscure what is naturally clear are referred to the version given by Bourbaki in [16, Chapter 2, $\S 3$, Theorem 1]. Using the Mittag-Leffler theorem and Corollary 3.2 , we now obtain the following result.

THEOREM 3.3. Assume that there exists a discontinuous character on some Fréchet algebra $A$, commutative or not. Then $\lim \operatorname{proj}\left(\mathbf{C}^{p_{n}}, F_{n}\right) \neq \varnothing$ for every projective system $\left(\mathbf{C}^{p_{n}}, F_{n}\right)_{n \geqslant 1}$, where $F_{n}: \mathbf{C}^{p_{n+1}} \rightarrow \mathbf{C}^{p_{n}}$ is entire for each $n \geqslant 1$.

Proof. We may assume that $A$ is unital. Set $\mathfrak{m}=\operatorname{Ker} \chi$, where $\chi$ is a discontinuous character on $A$, and if $a=\left(a_{1}, \ldots, a_{p}\right) \in A^{p}$ set $\chi_{p}(a)=$ $\left(\chi\left(a_{1}\right), \ldots, \chi\left(a_{p}\right)\right)$. Set $q_{1}=0$ and $q_{n}=p_{1}+\cdots+p_{n-1}$ for $n \geqslant 2$. Denote by $E_{n}$ the cartesian product $A^{p_{n}} \times \mathfrak{m}^{q_{n}}$ where $A$ is equipped with the given topology and where $m$ is equipped with the discrete topology. Note that $m$ is homeomorphic to a complete metrizable space so that $E_{n}$ is a complete metrizable space for every $n \geqslant 1$.

Now consider for every $n \geqslant 1$ the map

$$
\begin{gathered}
\Theta_{n}: E_{n+1} \rightarrow E_{n} \\
\left(a, x_{1}, \ldots, x_{q_{n+1}}\right) \rightarrow\left(F_{n}(a)+\left(x_{q_{n}+1}, \ldots, x_{q_{n+1}}\right), x_{1}, \ldots, x_{q_{n}}\right) .
\end{gathered}
$$

Since $q_{n+1}=q_{n}+p_{n}$, the above map is well defined. Since $\mathfrak{m}^{q_{n+1}}$ is equipped with the discrete topology and since $F_{n}: A^{p_{n+1}} \rightarrow A^{p_{n}}$ is continuous, we see that $\Theta_{n}$ is continuous. But $\mathfrak{m}^{p_{n}}$ is dense in $A^{p_{n}}$, so that $\Theta_{n}\left[\{a\} \times \mathfrak{m}^{q_{n+1}}\right]$ is dense in $E_{n}$ for each $n \geqslant 1$ and each $a \in A^{p_{n+1}}$. It follows from the MittagLeffler theorem that $\lim \operatorname{proj}\left(E_{n}, \Theta_{n}\right) \neq \varnothing$. Let $\left(u_{n}\right)_{n \geqslant 1}$ be an element of $\lim \operatorname{proj}\left(E_{n}, \Theta_{n}\right)$. We have $u_{n}=\left(a_{n}, V_{n}\right)$ where $a_{n} \in A^{p_{n}}, V_{n} \in \mathfrak{m}^{q_{n}}$ and it follows from the definition of $\Theta_{n}$ that $a_{n}-F_{n}\left(a_{n+1}\right) \in \mathfrak{m}^{p_{n}}$ for every $n \geqslant 1$. Then $\chi_{p_{n}}\left(a_{n}\right)=\chi_{p_{n}}\left(F_{n}\left(a_{n+1}\right)\right)(n \geqslant 1)$. It follows from Corollary 3.2 that $\chi_{p_{n}}\left(F_{n}\left(a_{n+1}\right)\right)=F_{n}\left(\chi_{p_{n+1}}\left(a_{n+1}\right)\right)$ and if we set $Z_{n}=\chi_{p_{n}}\left(a_{n}\right)(n \geqslant 1)$ we see that $\left(Z_{n}\right)_{n \geqslant 1} \in \lim \operatorname{proj}\left(\mathbf{C}^{p_{n}}, F_{n}\right)$. This proves the theorem. 
Corollary 3.4. Assume that there exists for some $p$ a sequence $\left(F_{n}\right)_{n \geqslant 1}$ of entire functions from $\mathbf{C}^{p}$ into itself such that $\bigcap_{n \geqslant 1} F_{1} \circ \cdots \circ F_{n}\left(\mathbf{C}^{p}\right)=\varnothing$. Then all characters on Fréchet algebras are continuous.

Proof. Set $p_{n}=p$ for every $n \geqslant 1$. Then $\pi_{1}\left(\lim \operatorname{proj}\left(\mathbf{C}^{p_{n}}, F_{n}\right)\right) \subset$ $\bigcap_{n \geqslant 1} \mathrm{~F}_{1} \circ \cdots \circ \mathrm{F}_{n}\left(\mathrm{C}^{p}\right)$, and the corollary follows from the theorem.

It follows easily from the big Picard theorem that $\pi_{1}\left(\lim \operatorname{proj}\left(\mathbf{C}, f_{n}\right)\right)$ equals either $\mathbf{C}$ or $\mathbf{C} \backslash\{\boldsymbol{\alpha}\}$, where $\{\boldsymbol{\alpha}\}$ is a singleton, for every sequence $\left(f_{n}\right)$ of nonconstant entire functions on $\mathbf{C}$ (and if the sequence $\left(f_{n}\right)$ contains a constant function the above set reduces to a singleton but is still nonempty). But the existence of a sequence $\left(F_{n}\right)$ satisfying the condition of Corollary 3.4 seems to be an open problem for every $p \geqslant 2$. The search for such a sequence was the motivation of the work presented in the following sections.

4. Properties of the range of nondegenerate entire functions from $\mathrm{C}^{p}$ into $\mathbf{C}^{p}$. Throughout the remainder of the paper we will denote by $\mathscr{H}\left(U, \mathbf{C}^{p}\right)$ the set of all holomorphic functions $F: U \rightarrow \mathbf{C}^{p}$ where $U$ is an open subset of $\mathbf{C}^{m}$ $(m \geqslant 1, p \geqslant 1)$. If $U$ is an open subset of $\mathbf{C}^{p}$ a function $F \in \mathscr{H}\left(U, \mathbf{C}^{p}\right)$ is said to be degenerate if its jacobian $J(F)$ vanishes everywhere.

In the following theorem, we collect some results concerning the range of nondegenerate entire mappings from $\mathbf{C}^{p}$ into itself. These results either are well known or follow easily from standard results of the theory of analytic functions of several complex variables.

THEOREM 4.1. Let $F: \mathbf{C}^{p} \rightarrow \mathbf{C}^{p}$ be a nondegenerate entire mapping.

(1) The convex hull of $F\left(\mathbf{C}^{p}\right)$ equals $\mathbf{C}^{p}$, and for every $z \in \mathbf{C}^{p}$ there exist $u_{1}, \ldots, u_{2 p} \in \mathbf{C}^{p}$ and $\lambda_{1} \geqslant 0, \ldots, \lambda_{2 p} \geqslant 0$ such that

$$
z=\sum_{i=1}^{2 p} \lambda_{i} F\left(u_{i}\right), \quad \sum_{i=1}^{2 p} \lambda_{i}=1, \quad J(F)\left(u_{i}\right) \neq 0 \quad(i=1, \ldots, 2 p) .
$$

(2) $F\left(\mathbf{C}^{p}\right)$ omits at most $p$ distinct hyperplanes.

If , further, $F$ is one-to-one, then the following properties hold.

(3) $F\left(\mathbf{C}^{p}\right)$ is pseudoconvex.

(4) The volume of $F\left(\mathbf{C}^{p}\right)$ is infinite.

(5) If $\mathbf{C}^{p} \backslash F\left(\mathbf{C}^{p}\right)$ is bounded, then $F$ is onto,

(6) If $F\left(\mathbf{C}^{p}\right)$ contains an open connected tube whose convex hull equals $\mathbf{C}^{p}$, then $F$ is onto.

Proof. Write $f=\left(f_{1}, \ldots, f_{p}\right)$, and set $U=\left\{u \in \mathbf{C}^{p} \mid J(F)(u) \neq 0\right\}$. Since $F$ is nondegenerate, $U$ is dense in $\mathbf{C}^{p}$, and it follows from [54, Chapter $1, \S \mathrm{C}$, Corollary 4] that $U$ is connected. Also it follows from the standard open mapping theorem [54, Chapter $1, \S \mathrm{B}$, Theorem 7] that $F(U)$ is open, so that its convex hull $V$ is open too. If $V \neq \mathbf{C}^{p}=\mathbf{R}^{2 p}$, there would exist a real hyperplane $H$ of $\mathbf{R}^{2 p}$ such that $H \cap V=\varnothing$ [15, Chapter II, §5, Theorem 1]. So there would exist a real linear form $L \neq 0$ on $\mathbf{R}^{2 p}$ such that $(L \circ F)(U)$ is bounded from above. Since $U$ is dense in $\mathbf{C}^{p}$, the pluriharmonic function $L \circ F$ would be bounded from above on $\mathbf{C}^{p}$, hence constant. So $F\left(\mathbf{C}^{p}\right)$ would be contained in a real hyperplane, which contradicts the fact that $F(U)$ is open. We thus see that the convex hull $V$ of $F(U)$ equals $\mathbf{C}^{p}$. 
Since $F(U)$ is connected, it follows from [15, Chapter II, §2, exercise 9] that for every $z \in V=\mathbf{C}^{p}$ there exists $v_{1}, \ldots, v_{2 p} \in F(U)$ and $\lambda_{1} \geqslant 0, \ldots, \lambda_{2 p} \geqslant 0$ such that $\sum_{i=1}^{2 p} \lambda_{i}=1, z=\sum_{i=1}^{2 p} \lambda_{i} v_{i}$. This proves (1).

Assertion (2) is well known, and follows almost immediately from a classical result of Borel; Borel proved in 1897 [14] that if $\exp g_{1}+\cdots+\exp g_{k} \equiv 0$, where $g_{1}, \ldots, g_{k}$ are entire functions over $\mathbf{C}$, then $g_{i}-g_{j}$ is constant for some $i \neq j$. Borel's theorem extends trivially to entire functions from $\mathbf{C}^{q}$ into $\mathbf{C}$, and it is easy to deduce from this extension of Borel's theorem the fact that if the range of an entire mapping $G: \mathbf{C}^{q} \rightarrow \mathbf{C}^{p}$ omits $p+1$ distinct hyperplanes, then $G\left(\mathbf{C}^{q}\right)$ is contained in a hyperplane. We refer to Nishino [79] for details. In fact assertion (2) seems to have been rediscovered many times (including by the authors when $p=2$ ). It seems that it was explicitly stated for the first time by Green [50], in terms of analytic mappings $G: \mathbf{C}^{q} \rightarrow P_{p}(\mathbf{C})$. Green proved also in [50] that any analytic mapping $G: \mathbf{C}^{q} \rightarrow P_{p}(\mathbf{C})$ which omits $2 p+1$ hyperplanes in general position is constant, which solved a problem raised by Chern [24] and Wu [109], but this result, which follows also from Borel's theorem, appears to have been obtained in 1944 by Dufresnoy [36]. Borel's theorem was extended by H. Cartan in 1928, in a paper [22] where he studied holomorphic maps from a punctured disc into $P_{n}(\mathbf{C})$, see $[109,110]$ for details and comments about the connections between these results and Ahlfors theory [2].

Now assume that $F$ is one-to-one. Then $F^{-1}: F\left(\mathbf{C}^{p}\right) \rightarrow \mathbf{C}^{p}$ is an analytic mapping. We write $F^{-1}=\left(g_{1}, \ldots, g_{p}\right)$. Then the function $\varphi: u \rightarrow$ sup $\log \left|g_{i}(u)\right|$ is plurisubharmonic over $F\left(\mathbf{C}^{p}\right)$. For $a \in \mathbf{R}$, set $W_{a}=\{u \in$ $\left.F\left(\mathbf{C}^{p}\right) \mid \varphi(u) \leqslant a\right\}$. Then $W_{a}=F\left(\Delta_{a}\right)(a \in \mathbf{R})$, where $\Delta_{a}=\left\{\left(z_{1}, \ldots, z_{p}\right) \in\right.$ $\left.\mathbf{C}^{p}|\sup | z_{i} \mid \leqslant e^{a}\right\}$. So $W_{a}$ is compact for every $a \in \mathbf{R}$, and the fact that $F\left(\mathbf{C}^{p}\right)$ is pseudoconvex follows from [56, Theorem 2.6.7]. This proves (3).

Now denote by $m$ the Lebesgue measure on $\mathbf{C}^{p}=\mathbf{R}^{2 p}$. If $\Omega$ is an open subset of $\mathrm{C}^{p}$ we have $\int_{F(\Omega)} d m=\int_{\Omega}|J(F)(z)|^{2} d m$, since the jacobian of $F: \mathbf{R}^{2 p} \rightarrow \mathbf{R}^{2 p}$ is the square of the modulus of the jacobian of $F$ considered as a function from $\mathbf{C}^{p}$ into $\mathbf{C}^{p}$. Hence $\int_{F\left(\mathbf{C}^{p}\right)} d m=\int_{\mathbf{R}^{2 p}}|J(F)(z)|^{2} d m$. But it follows from the subharmonicity of $|J(F)|$ that $J(F) \equiv 0$ if $\iint_{\mathbf{R}^{2 p} \mid}|J(F)(z)|^{2} d m$ were finite, which is impossible. So $m\left(F\left(\mathbf{C}^{p}\right)\right)=+\infty$, and (4) holds.

Note that the obvious change of variables given above shows that analytic maps from $\mathbf{C}^{p}$ into $\mathbf{C}^{p}$ of jacobian identically equal to 1 are volume preserving.

Now assume that $\mathbf{C}^{p} \backslash F\left(\mathbf{C}^{p}\right)$ is bounded. Then $F^{-1}$ is defined and analytic over $\mathbf{C}^{p} \backslash \Delta$, where $\Delta$ is some polydisc. It follows then from a basic theorem of Hartogs [56, Theorem 2.3.2] that the functions $g_{1}, \ldots, g_{p}$ possess analytic extensions to the whole of $\mathbf{C}^{p}$. So $F^{-1}$ possesses an analytic extension $G$ to $\mathbf{C}^{p}$ and $F \circ G$ is the identity map, so that $F$ is onto. This proves (5).

Similarly, if $F\left(\mathbf{C}^{p}\right)$ contains an open connected tube $L$, it follows from [56, Theorem 2.5.10] that $F^{-1}$ possesses an analytic extension to the convex hull $V$ of $L$. So $F$ is onto if $V=C^{p}$, which proves (6).

Some interesting information is obtained in a recent paper of Nishimura [77], where the author exhibits a one-to-one entire mapping $G: \mathbf{C}^{p} \rightarrow \mathbf{C}^{p}$ such that $G\left(\mathbf{C}^{p}\right) \neq H\left(\mathbf{C}^{p}\right)$ for every one-to-one entire mapping $H: \mathbf{C}^{p} \rightarrow \mathbf{C}^{p}$ whose jacobian is constant. In this paper, the author mentions an unpublished 
result of Ueda which shows that the interior of $\mathbf{C}^{p} \backslash F\left(\mathbf{C}^{p}\right)$ is pseudoconvex for every one-to-one entire mapping $F: \mathbf{C}^{p} \rightarrow \mathbf{C}^{p}$, a result much deeper than assertion (3). In another paper [76], Nishimura exhibited a one-to-one entire mapping $F: \mathbf{C}^{2} \rightarrow \mathbf{C}^{2}$ whose range avoids a neighborhood of a complex line (see §6), but the existence of one-to-one entire mappings from $\mathbf{C}^{2}$ into itself whose ranges avoid two distinct complex lines seems to be an open problem [77]. When $p=1$, assertion (2) reduces to the little Picard theorem, and the big Picard theorem also can be obtained by using Borel's theory [14].

Nevanlinna's theory [75] gives much more precise information about the distribution of values of entire functions of one complex variable, and Borel's theorem about sums of exponentials can be refined by Nevanlinna's methods, based upon the lemma of the logarithmic derivative (see also [75]). These methods can be extended to the study of analytic mappings $\mathbf{C}^{q} \rightarrow \mathbf{C}^{p}$, see Vitter [106]. In the same circle of ideas, value distribution theory gives some classes of sets, much more general than the union of $p+1$ distinct hyperplanes, which meet the range of any nondegenerate entire mapping $\mathbf{C}^{p} \rightarrow \mathbf{C}^{p}$ (see Carlson and Griffiths [21], Griffiths [51], Gruman [52]).

Sibony and Pit-Mann Wong showed in [98] that for each $\varepsilon>0$ there exists an entire, one-to-one mapping $F_{\varepsilon}: \mathbf{C}^{2} \rightarrow \mathbf{C}^{2}$ of jacobian 1 such that $F_{\varepsilon}\left(\mathbf{C}^{2}\right)$ is not dense in $\mathbf{C}^{2}$ and such that $\left|F_{\varepsilon}(u)\right| \leqslant c_{\varepsilon} \exp \left(|u|^{\varepsilon}\right)$ for every $u \in \mathbf{C}^{2}$, where $c_{\varepsilon}$ is a positive constant (we set $\left|\left(z_{1}, \ldots, z_{p}\right)\right|=\sup _{i \leqslant p}\left|z_{i}\right|$ for each element $z=\left(z_{1}, \ldots, z_{p}\right)$ of $\left.\mathbf{C}^{p}\right)$. In the other direction they showed that if a nondegenerate entire mapping $F=\left(f_{1}, \ldots, f_{p}\right)$ from $\mathbf{C}^{p}$ into itself satisfies $\left|f_{i}(u)\right| \leqslant$ $\exp \left(c_{i}[\log |u|]^{1+\varepsilon_{i}}\right)(|u| \geqslant 1, i=1, \ldots, p-1)$, where $c_{1}, \ldots, c_{p-1}$ are nonnegative constants and where $\varepsilon_{1}, \ldots, \varepsilon_{p-1}$ are positive constants such that $\varepsilon_{1}$ $+\cdots+\varepsilon_{p-1} \leqslant 1$, then $\mathbf{C}^{p} \backslash F\left(\mathbf{C}^{p}\right)$ is of Lebesgue measure 0 .

A slightly stronger condition implies that $\mathbf{C}^{p} \backslash F\left(\mathbf{C}^{p}\right)$ has $\Gamma$-capacity zero in the sense of Ronkin [89]; see also [98, Remark 2.4] (on the other hand a minor assertion of [98, Proposition 1.1] is not correct; see the comments at the end of the next section). We have not heard about any other growth condition on an entire mapping $F$ : $\mathbf{C}^{p} \rightarrow \mathbf{C}^{p}$ which ensures that the range of $F$ is dense in $\mathbf{C}^{p}$. There are even very hard open problems about polynomial maps $\varphi: \mathbf{C}^{p} \rightarrow \mathbf{C}^{p}$ whose jacobian nowhere vanishes. It is known that $\mathbf{C}^{p} \backslash \varphi\left(\mathbf{C}^{p}\right)$ is an algebraic variety of codimension at least two [49, Theorem 1] and that $\varphi$ is onto if $p=2, d^{0} \varphi \leqslant 100$ (see Moh [74]), or if $d^{0} \varphi \leqslant 2$ for any $p$ (Wang [108]), but it is still unknown for all $p \geqslant 2$, despite various claims of the contrary, whether $\varphi$ has to be onto. See the discussion of this problem, often called the jacobian conjecture, given by Bass, Connell and Wright in [10].

If $F: \mathbf{C}^{p} \rightarrow \mathbf{C}^{p}$ is a one-to-one entire mapping, then $F\left(\mathbf{C}^{p}\right)$ is indeed diffeomorphic to $\mathbf{C}^{p}$ and pseudoconvex. There exist open pseudoconvex subsets $U$ of $\mathbf{C}^{p}$ which are diffeomorphic to $\mathbf{C}^{p}$ and which do not possess any nonconstant bounded analytic functions, which are not biholomorphically equivalent to $\mathbf{C}^{p}$; see Diederich and Sibony [34].

On the other hand "strictly parabolic manifolds" are biholomorphically equivalent to $\mathbf{C}^{p}$, so that open subsets of $\mathbf{C}^{p}$ biholomorphically equivalent to $\mathbf{C}^{p}$ can be characterized by means of the Monge-Ampère equation, see the recent papers of Burns [18] and Stoll [104] for further information. 
5. Uniform convergence on compact sets of injective holomorphic maps. We set $\left|\left(z_{1}, z_{2}, \ldots, z_{p}\right)\right|=\sup _{i \leqslant p}\left|z_{i}\right|$ for every element $\left(z_{1}, \ldots, z_{p}\right)$ of $\mathbf{C}^{p}$, and if $U$ is an open subset of $\mathbf{C}^{p}$ and $V$ an open subset of $\mathbf{C}^{q}$, we denote by $\sigma_{U}$ the topology of uniform convergence on compact subsets of $U$ on the set $\mathscr{H}(U, V)$ of all holomorphic functions from $U$ into $V$. Note that if $W$ is an open subset of $\mathbf{C}^{r}$ the composition of maps is a continuous map from $\left(\mathscr{H}(V, W), \sigma_{V}\right) \times$ $\left(\mathscr{H}(U, V), \sigma_{U}\right)$ into $\left(\mathscr{H}(U, W), \sigma_{U}\right)$.

In particular if $F_{n} \underset{\sim}{\rightarrow} F$ and if $\left|u_{n}-u\right|_{n \rightarrow \infty} 0$ then $\left|F_{n}\left(u_{n}\right)-F(u)\right|_{n \rightarrow \infty} 0$. If $K$ is a compact subset of an open subset $U$ of $\mathbf{C}^{p}$ we set $\|F\|_{K}=$ $\sup _{u \in K}|F(u)|$ for every $F \in \mathscr{H}\left(U, \mathbf{C}^{q}\right)$. In order to state the next result with the generality we need we have to introduce the following notion.

Definition 5.1. Denote by $\mathscr{E}_{p}$ the set of all pairs $(F, U)$, where $U$ is an open subset of $\mathbf{C}^{p}$ and $F: U \rightarrow \mathbf{C}^{p}$ an analytic function. We will say that a sequence $\left(F_{n}, U_{n}\right)_{n \geqslant 1}$ of elements of $\mathscr{E}_{p}$ converges uniformly on compact sets to an element $(F, U)$ of $\mathscr{E}_{p}$, and we will write $\left(F_{n}, U_{n}\right) \stackrel{\sigma}{\rightarrow}(F, U)$ if for every compact subset $K$ of $U$ the following conditions are satisfied.

(1) There exists an integer $n(K)$ such that $K \subset U_{n}$ for every $n \geqslant n(K)$.

(2) The sequence $\left(\left\|F_{n}-F\right\|_{K}\right)_{n \geqslant n(K)}$ converges to 0 .

A well-known theorem [13, Chapter 8, Theorem 9] shows that if $U$ is a connected open subset of $\mathbf{C}^{p}$, and if $\left(F_{n}, U\right) \stackrel{\sigma}{\rightarrow}(F, U)$, where all the functions $F_{n}$ are one-to-one, then either $F$ is one-to-one or $F$ is degenerate. Also $\mathrm{H}$. Cartan showed in [23, Lemma 5] in the case where $p=2$ that if $\left(F_{n}, U\right) \stackrel{\sigma}{\rightarrow}(I, U)$, where $I$ is the identity map, then $F_{n} \mid K$ is eventually one-to-one for every compact subset $K$ of $U$ and that $L$ is eventually contained in $F_{n}(U)$ for every compact subset $L$ of $U$. The following theorem is a (certainly well-known) extension of these results.

THEOREM 5.2. Let $\left(F_{n}, U_{n}\right)_{n \geqslant 1}$ be a sequence of elements of $\mathscr{E}_{p}$ and let $(F, U) \in \mathscr{E}_{p}$, where $U$ is connected, such that $\left(F_{n}, U_{n}\right) \underset{n \rightarrow \infty}{\stackrel{\sigma}{\rightarrow}}(F, U)$. If $F_{n}$ is one-to-one over $U_{n}$ for every $n \geqslant 1$ and if $F$ is nondegenerate then $F$ is one-to-one and

$$
\left(F_{n}^{-1}, F_{n}\left(U_{n}\right)\right) \underset{n \rightarrow \infty}{\stackrel{\sigma}{\rightarrow}}\left(F^{-1}, F(U)\right) .
$$

Proof. It follows from a standard result mentioned above that $F \mid W$ is one-to-one for every relatively compact open connected subset $W$ of $U$, so that $F$ is one-to-one over $U$, since $U$ is arcwise connected. Also it follows from [13, Chapter 8, Theorem 7] that $J(F)(a) \neq 0$ for every $a \in U$. Fix $b \in F(U)$ and let $\Omega_{b}$ be an open neighborhood of $F^{-1}(b)$ which is relatively compact in $U$. Then $\Omega_{b} \subset U_{n}$ eventually and it follows from [13, Chapter 3, Theorem 3] that there exists an integer $n(b)$ and an open neighborhood $V(b)$ of $b$ in $F(U)$ such that $V(b) \subset F_{n}\left(\Omega_{b}\right)$ for every $n \geqslant n(b)$. Let $L$ be a compact subset of $F(U)$. We can find a finite family $b_{1}, \ldots, b_{k}$ of elements of $L$ such that $L \subset V\left(b_{1}\right) \cup \cdots \cup V\left(b_{k}\right)$ so that $L \subset F_{n}\left(\Omega_{b_{1}} \cup \cdots \cup \Omega_{b_{k}}\right) \subset F_{n}\left(U_{n}\right)$ for every $n \geqslant n(L)$, where $n(L)=\max \left(n\left(b_{1}\right), \ldots, n\left(b_{k}\right)\right)$.

Note that $\Omega_{b_{1}} \cup \cdots \cup \Omega_{b_{k}}$ is a relatively compact subset of $U$ so thàt $\cup_{n \geqslant n(L)} F_{n}^{-1}(L)$ is bounded. Now let $W$ be an open relatively compact subset 
of $F(U)$. There exists an integer $m$ and an open relatively compact subset $\Omega$ of $U$ containing $F^{-1}(\bar{W})$ such that $\Omega \subset U_{n}$ for every $n \geqslant m$ and such that $\left[\bigcup_{n \geqslant m} F_{n}^{-1}(W)\right]^{-} \subset \Omega$.

Set $G_{n}=F_{n}\left|\Omega, H_{n}=F_{n}^{-1}\right| W(n \geqslant m), G=F\left|\Omega, H=F^{-1}\right| W$. The family $\left(H_{n}\right)_{n \geqslant m}$ is normal. Let $\left(H_{n_{t}}\right)_{i \geqslant 1}$ be any subsequence of the sequence $\left(H_{n}\right)_{n \geqslant m}$ which is $\sigma_{W}$ convergent, and denote by $\Phi$ its limit. Then

$$
\Phi \in \mathscr{H}(W, \Omega), \quad H_{n_{i}} \underset{i \rightarrow \infty}{\stackrel{\sigma_{W}}{\rightarrow}} \Phi, \quad G_{n_{i}} \underset{i \rightarrow \infty}{\stackrel{\sigma_{\Omega}}{\rightarrow}} G
$$

so that

$$
G_{n_{i}} \circ H_{n_{i}} \underset{i \rightarrow \infty}{\stackrel{\sigma_{W}}{\rightarrow}} G \circ \Phi .
$$

So $\mathrm{G} \circ \Phi$ is the identity map on $W$, which means that $\Phi=H=F^{-1} \mid W$. Since $\mathscr{H}\left(W, \mathbf{C}^{p}\right)$ is homeomorphic to a metric space, this suffices to show that $H_{n} \underset{n \rightarrow \infty}{\stackrel{\sigma_{W}}{\rightarrow}} H$, and the sequence $\left(\left\|F^{-1}-F_{n}^{-1}\right\|_{L}\right)_{n \geqslant m}$ converges to zero for every compact subset $L$ of $W$. Since each compact subset of $F(U)$ possesses an open relatively compact neighborhood in $F(U)$, the theorem follows.

Denote by $\operatorname{Aut}\left(\mathbf{C}^{p}\right)$ the group of all analytic isomorphisms of $\mathbf{C}^{p}$ onto itself. We see in particular that the map $\Theta \rightarrow \Theta^{-1}$ is $\sigma$-continuous on $\operatorname{Aut}\left(\mathbf{C}^{p}\right)$. Note that $\operatorname{Aut}\left(\mathbf{C}^{p}\right)$ is homeomorphic to a complete metrizable group; to see this use the distance $\delta\left(\Theta_{1}, \Theta_{2}\right)=d\left(\Theta_{1}, \Theta_{2}\right)+d\left(\Theta_{1}^{-1}, \Theta_{2}^{-1}\right)$ where $d$ is a distance defining the $\boldsymbol{\sigma}$-topology on $\mathscr{H}\left(\mathbf{C}^{p}, \mathbf{C}^{p}\right)$ for which $\mathscr{H}\left(\mathbf{C}^{p}, \mathbf{C}^{p}\right)$ is complete.

The following corollary suggests a new method of constructing entire one-to-one functions from $\mathrm{C}^{p}$ into itself with nondense range.

COROLlaRY 5.3. Let $\left(\Theta_{n}\right)_{n \geqslant 1}$ be a sequence of elements of $\operatorname{Aut}\left(\mathbf{C}^{p}\right)$ and let $F \in \mathscr{H}\left(\mathbf{C}^{p}, \mathbf{C}^{p}\right)$ such that $\Theta_{n} \underset{n \rightarrow \infty}{\stackrel{\sigma}{\rightarrow}} F$. If $F$ is nondegenerate, we have the following properties.

(1) $F$ is one-to-one.

(2) $F\left(\mathbf{C}^{p}\right)$ is a Runge domain.

(3) $\Theta_{n}^{-1}(z)$ converges uniformly to $F^{-1}(z)$ over compact subsets of $F\left(\mathbf{C}^{p}\right)$.

(4) $\left|\Theta_{n}^{-1}(z)\right|_{n \rightarrow \infty} \infty$ uniformly over $\mathbf{C}^{p} \backslash F\left(\mathbf{C}^{p}\right)$.

Proof. Assertions (1) and (3) follow directly from the theorem. So the sequence $\left(F \circ \Theta_{n}^{-1}\right)$ converges uniformly to the identity map over compact subsets of $F\left(\mathbf{C}^{p}\right)$. Set $U=F\left(\mathbf{C}^{p}\right)$ and let $f \in \mathscr{H}(U, \mathbf{C})$. Then $f \circ F \circ \Theta_{n}^{-1} \underset{n \rightarrow \infty}{\stackrel{\sigma_{U}}{\rightarrow}} f$. Since $f \circ F \circ \Theta_{n}^{-1}$ is entire for every $n \geqslant 1, F\left(\mathbf{C}^{p}\right)$ is a Runge domain [56, Definition 2.7.1]. Now assume that there exists $R>0$ such that for some strictly increasing family $\left(n_{i}\right)$ of integers we have $\left|\Theta_{n_{1}}^{-1}\left(z_{n_{t}}\right)\right| \leqslant R(i \geqslant 1)$ where $z_{n} \in \mathbf{C}^{p} \backslash F\left(\mathbf{C}^{p}\right)$ for every $i$. Taking a suitable subsequence if necessary, we may assume that $\Theta_{n_{i}}^{-1}\left(z_{n_{i}}\right) \rightarrow i \rightarrow \infty \beta$ for some $\beta \in \mathbf{C}^{p}$. But then $z_{n_{i}}=$ $\Theta_{n_{i}}\left(\Theta_{n_{1}}^{-1}\left(z_{n_{1}}\right)\right) \underset{i \rightarrow \infty}{\rightarrow} F(\beta)$, which is impossible since $\mathbf{C}^{p} \backslash F\left(\mathbf{C}^{p}\right)$ is closed, because $F$ is one-to-one. So $\left|\Theta_{n}^{-1}(z)\right|_{n \rightarrow \infty} \rightarrow \infty$ uniformly over $\mathbf{C}^{p} \backslash F\left(\mathbf{C}^{p}\right)$, which concludes the proof.

It is also possible to show that if $\left(F_{n}, U_{n}\right) \stackrel{\sigma}{\rightarrow}(F, U)$ and if $F$ is one-to-one, then $F_{n} \mid K$ is eventually one-to-one for every compact subset $K$ of $U$. This 
follows from the fact that if $\left(F_{n}, U_{n}\right) \stackrel{\sigma}{\rightarrow}(F, U)$ then any $a \in U$ such that $J(F)(a) \neq 0$ possesses a neighborhood $V_{a}$ such that $F_{n} \mid V_{a}$ is eventually one-to-one. Also if $\left(F_{n}, U_{n}\right) \stackrel{\sigma}{\rightarrow}(F, U)$, if $F$ is nondegenerate, and if $U$ is connected, then every compact subset $L$ of $F(U)$ is eventually contained in $F_{n}\left(U_{n}\right)$ (this follows from an argument used in the proof of Theorem 5.2). The above results can be proved using Kronecker integrals, as in Cartan's proof of [23, Lemma 5], but it is also possible to establish them just using Cauchy's inequalities and standard consequences of the fixed point theorem, see [44, §4].

Note in particular that if a sequence $\left(F_{n}\right)$ of elements of $\mathscr{H}\left(\mathbf{C}^{p}, \mathbf{C}^{p}\right)$ converges uniformly to the identity map on compact sets then there exists for every $u \in \mathbf{C}^{p}$ an integer $n(u)$ such that $u \in F_{n}\left(\mathbf{C}^{p}\right)$ for every $n \geqslant n(u)$. This contradicts an assertion of Sibony and Pit-Mann Wong [98, Proposition 1.1] in which they produce such a sequence $\left(F_{n}\right)$ whose range avoids a neighborhood of $(1,1)$ for each $n$.

6. Functional equations, dynamical systems and entire functions with nondense range. In this section we will use the classical method to construct nondegenerate entire functions from $\mathbf{C}^{p}$ into itself with nondense range. Such functions were constructed by Fatou [46] and then independently by Bieberbach [11], and Bieberbach's example is a one-to-one function of jacobian identically equal to 1 . But the credit for this discovery should go to Poincaré, who explicitly pointed out this phenomenon in his 1890 paper [85, p. 339] (without exhibiting a specific example), more than 40 years before Bieberbach. The key to all these constructions is a theorem about normal forms for local analytic automorphisms with repulsive fixed point. By local analytic automorphism we mean the germ $F$ of an analytic $\mathbf{C}^{p}$-valued map at a point $\alpha \in \mathbf{C}^{p}$ satisfying $J(F)(\alpha) \neq 0$.

If $F(\alpha)=\alpha$, then $\alpha$ is a fixed point for $F$ and the fixed point $\alpha$ is called repulsive if the eigenvalues $\lambda_{1}, \ldots, \lambda_{p}$ of the differential $F^{\prime}(\alpha)$ of $F$ at $\alpha$ satisfy inf $_{i \leqslant p}\left|\lambda_{i}\right|>1$. We have the following theorem.

THEOREM 6.1. Let $F$ be the germ of an analytic automorphism at $\alpha \in \mathbf{C}^{p}$ and assume that $\alpha$ is a repulsive fixed point for $F$. Then there exist a polynomial automorphism $B$ of $\mathbf{C}^{p}$ such that

$$
B(\alpha)=\alpha, \quad B^{-n} \underset{n \rightarrow \infty}{\stackrel{\sigma}{\rightarrow}} \alpha
$$

and a local automorphism $G$ of $\mathrm{C}^{p}$ at $\alpha$ such that $G(\alpha)=\alpha$ satisfying the relation $F \circ G=G \circ B$.

This result is an immediate consequence of Reich's papers $[87,88]$ about normal forms for local analytic automorphisms with repulsive fixed point at the origin.

It is straightforward to check that the polynomial "normal form" $B$ given by Reich in [88] satisfies $B^{-n}(z) \underset{n \rightarrow \infty}{\rightarrow} 0$ for every $z \in \mathbf{C}^{p}$, the convergence being uniform on compact subsets of $\mathbf{C}^{p}$. If the eigenvalues $\lambda_{1}, \ldots, \lambda_{p}$ have no "relations", i.e., if for $i \leqslant p, n_{1}, \ldots, n_{p} \geqslant 0, n_{1}+\cdots+n_{p} \geqslant 2$ we have $\lambda_{i} \neq \lambda_{1}^{n_{1}} \cdots \lambda_{p}^{n_{p}}$, then we can take $B=F^{\prime}(0)$, or we can take $B$ to be a Jordan form of $F^{\prime}(0)$. 
COROLlaRY 6.2. Let $U$ be an open subset of $\mathbf{C}^{p}$ and assume that there exists an analytic function $F: U \rightarrow U$ which possesses a repulsive fixed point $\alpha$ in $U$. Then there exists an entire function $G: \mathbf{C}^{p} \rightarrow U$, with $J(G)(\alpha) \neq 0, G(\alpha)=\alpha$ satisfying the functional equation $F \circ G=G \circ B$, where $B$ is a polynomial automorphism of $\mathbf{C}^{p}$ such that $B^{-n} \underset{n \rightarrow \infty}{\stackrel{\sigma}{\rightarrow}} \alpha$. Moreover

$$
\begin{aligned}
& G\left(\mathbf{C}^{p}\right)=\left\{z \in U \mid z=F^{n}\left(u_{n}\right)(n \geqslant 1)\right. \text { for some } \\
& \text { sequence } \left.\left(u_{n}\right) \subset U \text { with } u_{n \rightarrow \infty} \alpha\right\} .
\end{aligned}
$$

Proof. Let $G$ be the germ at $\alpha$ of an analytic automorphism with fixed point at $\alpha$ given by Theorem 6.1. In terms of germs of analytic automorphisms at $\alpha$, we have the identity $F \circ G \circ B^{-1}=G$. Let $V$ be an open neighborhood of $\alpha$ contained in the domain of $G$ such that $G(V) \subset U, B^{-1}(V) \subset V$ and such that $\left(F \circ G \circ B^{-1}\right)(z)=G(z)(z \in V)$.

Set $V_{n}=B^{n}(V)$. Then $F^{n} \circ G \circ B^{-n}$ is defined and analytic over $V_{n}$. Now let $K$ be a compact subset of $\mathbf{C}^{p}$. There exists an integer $n_{K}$ such that $B^{-n}(K) \subset$ $V$, hence $K \subset V_{n}$ for every $n \geqslant n_{K}$. If $n \geqslant n_{K}$ we have

$$
\begin{aligned}
F^{n+1} \circ G \circ B^{-n-1}(z) & =F^{n}\left[\left[F \circ G \circ B^{-1}\right]\left[B^{-n}(z)\right]\right] \\
& =F^{n} \circ G \circ B^{-n}(z) \quad(z \in K) .
\end{aligned}
$$

Hence $\left(F^{n} \circ G \circ B^{-n}, V_{n}\right)$ trivially $\sigma$-converges to an analytic extension of $G$ to the whole of $\mathbf{C}^{p}$. We may denote this extension by $G$, and $G\left(\mathbf{C}^{p}\right) \subset U$. Since $F[G(z)]=G[B(z)]\left(z \in B^{-1}(V)\right)$, the functional equation $F \circ G=G \circ B$ holds. Hence $F^{n} \circ G=G \circ B^{n}(n \geqslant 1)$. If $u=G(z)$ for some $z \in \mathbf{C}^{p}$, then

$$
u=\left(G \circ B^{n}\right)\left[B^{-n}(z)\right]=F^{n}\left[G\left[B^{-n}(z)\right]\right],
$$

so that $u=F^{n}\left(x_{n}\right)(n \geqslant 1)$, where $x_{n}=G\left[B^{-n}(z)\right]_{n \rightarrow \infty}^{\rightarrow} \alpha$. Conversely if $u=F^{n}\left(x_{n}\right)(n \geqslant 1)$, where $x_{n} \underset{n \rightarrow \infty}{\rightarrow} \alpha$, then $x_{n} \in G(V)$ for some $n$, so that $x_{n}=\left(G \circ B^{-n}\right)\left(y_{n}\right)$, where $y_{n} \in V_{n}$, and

$$
u=\left(F^{n} \circ G \circ B^{-n}\right)\left(y_{n}\right)=G\left(y_{n}\right) \in G\left(\mathbf{C}^{p}\right) .
$$

This concludes the proof of the corollary.

COROLlaRy 6.3. Let $U$ be an open subset of $\mathbf{C}^{p}$. There exists a nondegenerate (resp. with nonvanishing jacobian, resp. one-to-one) entire function $G: \mathbf{C}^{p} \rightarrow U$ if and only if there exists an analytic (resp. with nonvanishing jacobian, resp. one-to-one) function $F: U \rightarrow U$ which possesses a repulsive fixed point in $U$.

Proof. Assume that such a mapping $G$ : $\mathbf{C}^{p} \rightarrow U$ does exist, and let $\beta \in \mathbf{C}^{p}$ such that $J(G)(\beta) \neq 0$. Set $\alpha=G(\beta)$. There exists an affine automorphism $\Theta$ of $\mathrm{C}^{p}$ such that $\Theta(\alpha)=\beta, \Theta^{\prime}=2\left[G^{\prime}(\beta)\right]^{-1}$. Set $F=G \circ \Theta \mid U$. Then $F(U) \subset$ $U, F(\alpha)=\alpha, F^{\prime}(\alpha)=2 I$, where $I$ is the identity map on $\mathbf{C}^{p}$, and $\alpha$ is a repulsive fixed point for $U$. Clearly, $F$ has nonvanishing jacobian (resp. is one-to-one) if $G$ is such that the condition is necessary.

Conversely assume that such a function $F: U \rightarrow U$ exists. The function $G$ given by Corollary 6.2 is an entire function over $\mathbf{C}^{p}$ whose range is contained in $U$, and $J(G)(\alpha) \neq 0$ so that $G$ is nondegenerate. We have $F^{n} \circ G \circ B^{-n}=G$, and $J(G)$ never vanishes on some neighborhood $W$ of $\alpha$. Let $z \in \mathbf{C}^{p}$. Then 
$B^{-n}(z) \in W$ for some $n \geqslant 1$ so that

$$
J(G)(z)=J\left(F^{n}\right)\left[G \circ B^{-n}(z)\right] J(G)\left[B^{-n}(z)\right] J\left[B^{-n}\right](z) \neq 0
$$

if $J(F)$ never vanishes over $U$. Also $G \mid \Omega$ is one-to-one for some neighborhood $\Omega$ of $\alpha$, and if $G\left(z_{1}\right)=G\left(z_{2}\right)$ then $B^{-n}\left(z_{1}\right) \in \Omega, B^{-n}\left(z_{2}\right) \in \Omega$ for some $n \geqslant 1$, hence $G\left(B^{-n}\left(z_{1}\right)\right)=G\left(B^{-n}\left(z_{2}\right)\right), B^{-n}\left(z_{1}\right)=B^{-n}\left(z_{2}\right), z_{1}=z_{2}$ if $F$ is one-to-one over $U$. This concludes the proof of Corollary 6.3.

Corollary 6.3 gives a "dynamical" characterization of open subsets of $\mathbf{C}^{p}$ which contain the range of a nondegenerate entire function from $\mathbf{C}^{p}$ into itself. Such a statement was not in print, as far as the authors are aware.

The following corollary gives a characterization of open subsets of $\mathbf{C}^{p}$ which are biholomorphically equivalent to $\mathbf{C}^{p}$. It is a special case of a more general result of Kato [60], which deals with complex spaces instead of open subsets of $\mathbf{C}^{p}$. We nevertheless give a proof, because Kato's argument involves a reference (reference [2] in [60]) which seems inaccurate.

Corollary 6.4. Let $U$ be an open subset of $\mathbf{C}^{p}$. Then there exists a one-to-one entire function $G: \mathbf{C}^{p} \rightarrow \mathbf{C}^{p}$ such that $G\left(\mathbf{C}^{p}\right)=U$ if and only if there exists an analytic automorphism $\Theta$ of $U$ which possesses a fixed point $\alpha \in U$ for which $\Theta^{-n}(z) \underset{n \rightarrow \infty}{\rightarrow} \alpha$ for every $z \in U$.

Proof. If such a one-to-one entire function $G$ exists, then $U$ is biholomorphically equivalent to $\mathbf{C}^{p}$ and such an analytic automorphism $\Theta$ exists for each $\alpha \in U$. Now assume that there exists an analytic automorphism $\Theta$ of $U$ satisfying the above condition for some $\alpha \in U$. Then $\alpha$ is a repulsive fixed point for $\Theta$. Let $G$ be the entire function on $\mathbf{C}^{p}$ given by Corollary 6.2. Then $G$ is one-to-one, and $z=\Theta^{n}\left[\Theta^{-n}(z)\right](n \geqslant 1)$, where $\Theta^{-n}(z) \underset{n \rightarrow \infty}{\rightarrow} \alpha$ for every $z \in U$, so that $G\left(\mathbf{C}^{p}\right)=U$. This proves the corollary.

We now wish to prove a weak form of Theorem 6.1, which is sufficient to solve the functional equation $F \circ G=G \circ F^{\prime}(0)$ in the cases which arise in the constructions of Fatou [46], Bieberbach [11], Sadullaev [93], and Kodaira [61]. The idea of getting $G$ as the uniform limit on compact sets of the sequence $F^{n} \circ\left[F^{\prime}(0)\right]^{-n}$ is certainly not new (see the discussion at the end of this section) but the proof given below, which was communicated to the authors by W. Rudin [91], is much shorter than all the proofs that we found in the literature (see for example Stehlé [101]).

THEOREM 6.5. Let $F$ be the germ of an invertible analytic map at the origin on $\mathrm{C}^{p}$. Assume that $F(0)=0$ and that the eigenvalues $\lambda_{1}, \ldots, \lambda_{p}$ of $F^{\prime}(0)$ satisfy $1<\left|\lambda_{1}\right| \leqslant \cdots \leqslant\left|\lambda_{p}\right|,\left|\lambda_{1}^{2}\right|>\left|\lambda_{p}\right|$. Then the sequence $\left(F^{\prime}(0)^{n} \circ F^{-n}\right)_{n \geqslant 1}$ converges uniformly on a neighborhood $\Omega$ of the origin to an analytic map $H: \Omega \rightarrow \mathbf{C}^{p}$ satisfying $H(0)=0, H^{\prime}(0)=I,\left(F^{\prime}(0) \circ H \circ F^{-1}\right)(z)=H(z)(z \in$ $\Omega)$.

Proof. Fix two real numbers $\mu$ and $\nu$ satisfying $1<\nu<\left|\lambda_{1}\right|, \mu>\left|\lambda_{p}\right|$, $\mu \nu^{-2}<1$. It follows from the theory of Jordan forms that there exists for every $\varepsilon>0$ a basis $\left(e_{1}, \ldots, e_{p}\right)$ of $\mathbf{C}^{p}$ such that $F^{\prime}(0)\left(e_{1}\right)=\lambda_{1} e_{1}, F^{\prime}(0)\left(e_{i}\right)=\lambda_{i} e_{i}+$ $\varepsilon_{t} e_{t-1}$ with $\left|\varepsilon_{t}\right| \leqslant \varepsilon(i \geqslant 2)$. It follows from this observation that there exists a norm $\|\cdot\|$ over $\mathbf{C}^{p}$ such that $\left\|F^{\prime}(0)\right\|<\mu,\left\|\left[F^{\prime}(0)\right]^{-1}\right\|<\nu^{-1}$. Denote by $S$ the 
open unit ball of $\left(\mathbf{C}^{p},\|\cdot\|\right)$. There exists $r>0$ such that $\left\|F^{-1}(z)\right\| \leqslant \nu^{-1}\|z\|$ for every $z \in r S$.

Set $\Omega=r S$ and denote by $\mathscr{E}$ the space of all bounded analytic mappings $\varphi: \Omega \rightarrow \mathbf{C}^{p}$ satisfying $\varphi(0)=0, \varphi^{\prime}(0)=I$. Then $\mathscr{E}$ is a complete metric space with respect to the norm $\|\varphi\|=\sup _{z \in \Omega}\|\varphi(z)\|$, and it follows from the chain rule that $F^{\prime}(0) \circ \varphi \circ F^{-1} \in \mathscr{E}$ for every $\varphi \in \mathscr{E}$ (the mapping $F^{\prime}(0) \circ \varphi \circ F^{-1}$ is well defined on $\Omega$ since $\left.F^{-1}(\Omega) \subset \Omega\right)$. Set $\Theta(\varphi)=F^{\prime}(0) \circ \varphi \circ F^{-1}(\varphi \in \mathscr{E})$.

Fix $\varphi_{1}, \varphi_{2} \in \mathscr{E}$. If $\left\|z_{0}\right\|=r$, the function $\xi \rightarrow\left(\varphi_{1}\left(\xi z_{0}\right)-\varphi_{2}\left(\xi z_{0}\right)\right) / \xi$ is defined and analytic on the open unit disc of the complex plane and vanishes at the origin. It follows from the Schwartz lemma that

$$
\left\|\varphi_{1}\left(\xi z_{0}\right)-\varphi_{2}\left(\xi z_{0}\right)\right\| \leqslant|\xi|^{2}\left\|\varphi_{1}-\varphi_{2}\right\| \quad(|\xi|<1)
$$

so that

$$
\left\|\varphi_{1}(z)-\varphi_{2}(z)\right\| \leqslant \frac{\|z\|^{2}}{r^{2}}\left\|\varphi_{1}-\varphi_{2}\right\| \quad(z \in \Omega) .
$$

Hence $\left\|\Theta\left(\varphi_{1}\right)-\Theta\left(\varphi_{2}\right)\right\| \leqslant\left\|F^{\prime}(0)\right\| \nu^{-2}\left\|\varphi_{1}-\varphi_{2}\right\| \leqslant \mu \nu^{-2}\left\|\varphi_{1}-\varphi_{2}\right\|$ for every $\varphi_{1}, \varphi_{2} \in \mathscr{E}$. Since $\mu \nu^{-2}<1$, it follows from the usual fixed point theorem that the equation $\Theta(\varphi)=\varphi$ has a unique solution $H$ in $\mathscr{E}$. Moreover

$$
\left\|\left[F^{\prime}(0)\right]^{n} \circ \varphi \circ F^{-n}-H\right\|=\left\|\Theta^{n}(\varphi)-H\right\|_{n \rightarrow \infty} 0
$$

for every $\varphi \in \mathscr{E}$. Since $I \in \mathscr{E}$, this achieves the proof of the theorem.

COROllary 6.6. Let $U$ be an open subset of $\mathbf{C}^{p}$ containing the origin and let $F: U \rightarrow U$ be an analytic mapping such that $F(0)=0$. If the eigenvalues $\left(\lambda_{1}, \ldots, \lambda_{p}\right)$ of $F^{\prime}(0)$ satisfy $1<\left|\lambda_{1}\right| \leqslant \cdots \leqslant\left|\lambda_{p}\right|,\left|\lambda_{1}\right|^{2}>\left|\lambda_{p}\right|$, then the sequence $\left(F^{n} \circ\left[F^{\prime}(0)\right]^{-n}\right)_{n \geqslant 1}$ converges uniformly on compact subsets of $\mathbf{C}^{p}$ to an entire mapping $G: \mathbf{C}^{p} \rightarrow U$ such that $G(0)=0, G^{\prime}(0)=I, F \circ G=G \circ F^{\prime}(0)$, and $G\left(\mathbf{C}^{p}\right)=\left\{z \in U \mid z=F^{n}\left(u_{n}\right)(n \geqslant 1)\right.$ for some sequence $\left(u_{n}\right) \subset U$ with $\left.u_{n} \underset{n \rightarrow \infty}{\rightarrow} 0\right\}$.

Proof. Let $\Omega$ and $H$ be as in Theorem 6.5, and set $V=H(\Omega)$. Then

$$
\left(\left[F^{\prime}(0)\right]^{n} \circ F^{-n}, \Omega\right) \stackrel{\sigma}{\rightarrow}(H, \Omega),
$$

so that $H$ is one-to-one, and it follows from Theorem 5.2 that

$$
\left(F^{n} \circ\left[F^{\prime}(0)\right]^{-n}, \Omega_{n}\right) \stackrel{\sigma}{\rightarrow}\left(H^{-1}, V\right),
$$

where $\Omega_{n}=\left[F^{\prime}(0)\right]^{n}\left[F^{-n}(\Omega)\right](n \geqslant 1)$. Let $K$ be a compact subset of $\mathbf{C}^{p}$. There exists $k \geqslant 1$ such that $\left[F^{\prime}(0)\right]^{-k}(K) \subset V$, so that the sequence $\left(F^{n} \circ\left[F^{\prime}(0)\right]^{-n-k}\right)_{n \geqslant 1}$ is uniformly convergent over $K$. So the sequence $\left(F^{n+k} \circ\left[F^{\prime}(0)\right]^{-n-k}\right)_{n \geqslant 1}$ is also uniformly convergent oyer $K$ and the sequence $\left(F^{n} \circ\left[F^{\prime}(0)\right]^{-n}\right)$ converges uniformly over compact subsets of $\mathbf{C}^{p}$ to an entire mapping $G: \mathbf{C}^{p} \rightarrow U$ such that $G \mid V=H^{-1}$. So $G(0)=0, G^{\prime}(0)=I$. The last assertion follows from the same arguments as in Corollary 6.2.

We now describe Fatou's example. In Fatou's case we consider the entire function $F:(x, y) \rightarrow\left(y, 2 x+4 x y-3 y^{2}\right)$. This function has $(0,0)$ and $(-1,-1)$ as repulsive fixed points (the eigenvalues of $F^{\prime}(0)$ at $(0,0)$ and $(-1,-1)$ are respectively $\pm \sqrt{2}$ and $1 \pm i$ ). There exists a nondegenerate entire function $G$ 
whose range is the set of all $z \in \mathbf{C}^{2}$ such that $z=F^{n}\left(z_{n}\right)(n \geqslant 1)$ where $z_{n} \rightarrow \infty(0,0)$. Moreover $G$ can be arranged to satisfy the functional equation $F \circ G=G \circ B$, where $B$ is the linear map $\left(\begin{array}{cc}\sqrt{2} & 0 \\ 0 & -\sqrt{2}\end{array}\right)$. The function $F$ is not one-to-one, since $F\left(t,-\frac{1}{2}\right)=\left(-\frac{1}{2},-\frac{3}{4}\right)(t \in \mathbf{C})$, but the "inverse map" $F^{-1}$ is defined for $u \neq-\frac{1}{2}$ by the formula $F^{-1}(u, v)=\left(v+3 u^{2} /(2+4 u)\right.$, $\left.u\right)$. There exists a neighborhood $V$ of $(-1,-1)$ stable under $F^{-1}$ such that $F^{-n}(z) \underset{n \rightarrow \infty}{\rightarrow}(-1,-1)(z \in V)$, and since $F^{-n}(z)$ is the unique element $z_{n}$ of $\mathbf{C}^{2}$ such that $F^{n}\left(z_{n}\right)=z$ for $z \in V$, we have $G\left(\mathbf{C}^{2}\right) \cap V=\varnothing$, so that $G\left(\mathbf{C}^{2}\right)$ is not dense in $\mathbf{C}^{2}$.

We now check that, despite some claims of the contrary, Fatou's function $G$ is not one-to-one, and that we even have $J(G)(z)=0$ for some $z \in \mathbf{C}^{2}$. Set $W=\left\{(x, y) \in \mathbf{R}^{2} \mid x \geqslant 0, y \geqslant 0\right\}$. Clearly, $F^{-1}(W) \subset W$. If $z=(x, y) \in \mathbf{C}^{2}$ set $F^{-n}(z)=\left(x_{n}, y_{n}\right)(n \geqslant 1)$. If $z=(x, y) \in W$ we have either $x \geqslant 2 y / 3$, hence $x_{1} \leqslant 3 x / 4$, or $x \leqslant 2 y / 3$, hence $x_{1} \leqslant y / 2$. So $\left|F^{-1}(z)\right| \leqslant|z|$ and $x_{1} \leqslant$ $3|z| / 4(z \in W)$. Hence

$$
x_{2} \leqslant \frac{3\left|F^{-1}(z)\right|}{4} \leqslant \frac{3|z|}{4}, \quad y_{2}=x_{1} \leqslant \frac{3|z|}{4}
$$

so that $\left|F^{-2}(z)\right| \leqslant 3|z| / 4$ and $F^{-n}(z) \underset{n \rightarrow \infty}{\rightarrow}(0,0)$ for every $z \in W$. Now if $z=(x, y) \in \mathbf{R}^{2}$ with $x \geqslant 0, y \geqslant-3 x^{2}$ then $F^{-1}(z) \in W$, so that $F^{-n}(z) \underset{n \rightarrow \infty}{\rightarrow} 0$. In particular if $y=-\frac{1}{2}, x \geqslant 1 / \sqrt{6}$ then $F^{-n}(z) \underset{n \rightarrow \infty}{\rightarrow} 0$, and hence $z \in G\left(\mathbf{C}^{2}\right)$.

Also $G$ satisfies the functional equation $F \circ G=G \circ B$, where $B$ is the linear $\operatorname{map}(x, y) \rightarrow(\sqrt{2} x,-\sqrt{2} y)$. For $t \geqslant 0$ set $z_{t}=\left(1+t,-\frac{1}{2}\right)$ so that $z_{t}=G\left(u_{t}\right)$ for some $u_{t} \in \mathbf{C}^{2}$. We have $\left(-\frac{1}{2},-\frac{3}{4}\right)=F\left(z_{t}\right)=G\left(B\left(u_{t}\right)\right)$, so that $G$ is not one-to-one since $B\left(u_{t}\right) \neq B\left(u_{t^{\prime}}\right)$ if $t, t^{\prime} \geqslant 0, t \neq t^{\prime}$. Moreover $J(F)\left(z_{t}\right)=0$, so that $J(G)\left(B\left(u_{t}\right)\right)=0$ for every $t \geqslant 0$.

Bieberbach's example [11], described by Bochner and Martin [13, Chapter 3] and by Stehlé $[101]$ uses the map $F:(x, y) \rightarrow(u, v)$ where $u=4 x+2 y^{5}-$ $5 y^{2}, v=4 y+2 u^{5}-5 u^{2}$.

This map is clearly an analytic automorphism of $\mathbf{C}^{2}$, the inverse map being the map $(u, v) \rightarrow(x, y)$ where $x=\left(u-2 y^{5}+5 y^{2}\right) / 4, \quad y=\left(v-2 u^{5}+\right.$ $\left.5 u^{2}\right) / 4$. We have $F(0,0)=(0,0), F(1,1)=(1,1), F^{\prime}(0,0)=\left(\begin{array}{ll}4 & 0 \\ 0 & 4\end{array}\right)=F^{\prime}(1,1)$. So there exist two entire one-to-one functions $G_{1}$ and $G_{2}$ such that

$$
\begin{aligned}
& G_{1}\left(\mathbf{C}^{2}\right)=\left\{z \in \mathbf{C}^{2} \mid F^{-n}(z) \underset{n \rightarrow \infty}{\rightarrow}(0,0)\right\}, \\
& G_{2}\left(\mathbf{C}^{2}\right)=\left\{z \in \mathbf{C}^{2} \mid F^{-n}(z) \underset{n \rightarrow \infty}{\rightarrow}(1,1)\right\} .
\end{aligned}
$$

So $G_{1}\left(\mathbf{C}^{2}\right) \cap G_{2}\left(\mathbf{C}^{2}\right)=\varnothing$ and, since $G_{1}\left(\mathbf{C}^{2}\right)$ and $G_{2}\left(\mathbf{C}^{2}\right)$ are open, neither $G_{1}\left(\mathbf{C}^{2}\right)$ nor $G_{2}\left(\mathbf{C}^{2}\right)$ is dense in $\mathbf{C}^{2}$. In fact $G_{1}\left(\mathbf{C}^{2}\right)$ and $G_{2}\left(\mathbf{C}^{2}\right)$ contain respectively a neighborhood of $(0,0)$ and a neighborhood of $(1,1)$, since these points are attractive fixed points for $F^{-1}$. The above function $G_{1}$, normalized to satisfy $G_{1}(0)=I$, and $F\left(G_{1}(z)\right)=G_{1}(4 z)\left(z \in \mathbf{C}^{2}\right)$, is the original example of Bieberbach. 

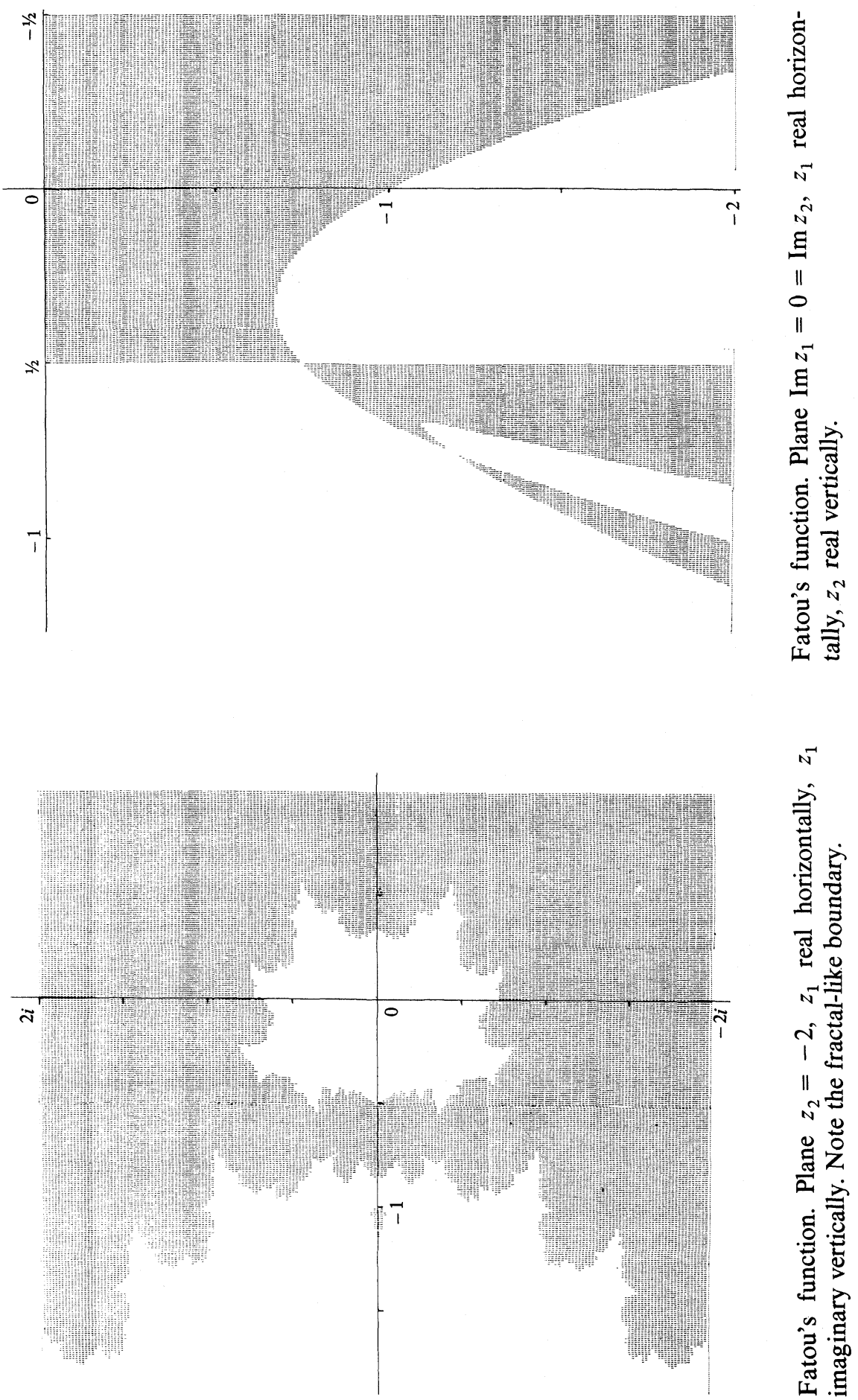
As suggested by computer pictures, the range of the function $G_{1}$ is rather "small". In fact, consider more generally a map $F_{k, j}$ of the form $(x, y) \rightarrow(u, v)$ with $u=k x+j(y), v=k y+j(u)$, where $j$ is a polynomial. If $j(0)=j^{\prime}(0)=0$ then $F_{k, j}(0,0)=(0,0), F_{k, j}(0,0)=\left(\begin{array}{cc}k & 0 \\ 0 & k\end{array}\right)$ so that $(0,0)$ is a repulsive fixed point for $F_{k, j}$ if $|k|>1$. Clearly, $F_{k, j}$ is an automorphism of $\mathbf{C}^{2}$ and so there exists an entire one-to-one function $G_{k, j}$ such that $G_{k, j}\left(\mathbf{C}^{2}\right)=\{z \in$ $\left.\mathrm{C}^{2} \mid F_{k, j}^{-n}(z) \underset{n \rightarrow \infty}{\rightarrow}(0,0)\right\}$. The above function $G_{1}$ has this form with $k=4$, $j(X)=2 X^{5}-5 X^{2}$. The following proposition shows in particular that $G_{1}\left(\mathbf{C}^{2}\right) \subset\left\{(u, v) \in \mathbf{C}^{2}|| u \mid>\max (2,|v|)\right\} \cap\left\{(u, v) \in \mathbf{C}^{2}|| v-2 u^{5}+5 u^{2} \mid<\right.$ $4 \max (2,|u|)\}$.

Proposition 6.7. Assume that $|k|>1$ and $j(0)=j^{\prime}(0)=0, d^{0} j \geqslant 2$. Let $K>0$ be such that $|j(z)| \geqslant(k+1)|z|(|z| \geqslant K)$. Then

(i) $|u|<\max (K,|v|)$ for every $(u, v) \in G_{k, j}\left(\mathbf{C}^{2}\right)$.

(ii) $|v-j(u)|<k \max (|u|, K)$ for every $(u, v) \in G_{k, j}\left(\mathbf{C}^{2}\right)$.

Proof. $F_{k, j}^{-1}$ is the map $(u, v) \rightarrow(x, y)$, where $x=k^{-1}(u-j(y)), y=$ $k^{-1}(v-j(u))$.

(i) If $|u| \geqslant K$ and $|u| \geqslant|v|$ then $|j(u)| \geqslant(k+1)|u|$, so

$$
|y| \geqslant k^{-1}(|j(u)|-|v|) \geqslant k^{-1}(|j(u)|-|u|) \geqslant|u|
$$

and so $|y| \geqslant|u| \geqslant K$. The same argument then gives $|x| \geqslant|y|$ and $|y| \geqslant K$. Thus, by induction, all the points $F_{k, j}^{-n}(u, v)=\left(x_{n}, y_{n}\right)$ satisfy $\left|x_{n}\right| \geqslant\left|y_{n}\right| \geqslant K$. Therefore $(u, v) \notin G_{k, j}\left(\mathbf{C}^{2}\right)$.

(ii) If $|v-j(u)| \geqslant k \max \{|u|, K\}$ then

$$
|y|=k^{-1}|v-j(u)| \geqslant \max (|u|, K),
$$

hence, as above, $|x| \geqslant|y| \geqslant K$. So $F_{k, j}^{-1}(u, v) \notin G_{k, j}\left(\mathbf{C}^{2}\right)$ and hence $(u, v) \notin$ $G_{k, j}\left(\mathbf{C}^{2}\right)$.

Note that the above proposition enables us to use the polynomial automorphism

$$
F=F_{2, X^{2}}:(x, y) \rightarrow\left(2 x+y^{2}, 2 y+\left(2 x+y^{2}\right)^{2}\right) .
$$

Then $(0,0)$ is a repulsive fixed point for $F$, with $F^{\prime}(0,0)=\left(\begin{array}{ll}2 & 0 \\ 0 & 2\end{array}\right)$. But the other fixed points are $(-1,-1),\left(-e^{2 i \pi / 3}, e^{i \pi / 3}\right),\left(-e^{-2 i \pi / 3}, e^{-i \pi / 3}\right)$ and at these points the eigenvalues of $F^{\prime}$ are $4 \pm \sqrt{12}$ so that these points are neither attractive nor repulsive. Nevertheless it follows from the proposition that

$$
\begin{aligned}
& G_{2, X^{2}}\left(\mathbf{C}^{2}\right) \subseteq\left\{(u, v) \in \mathbf{C}^{2}|| u \mid<\max (3,|v|)\right\} \\
& \cap\left\{(u, v) \in \mathbf{C}^{2}|| v-u^{2} \mid<2 \max (|u|, 3)\right\} .
\end{aligned}
$$

In particular $|v| \leqslant 15$ if $|u| \leqslant 3$ and $|u|<|v|<2|u|+|u|^{2}$ if $|u| \geqslant 3$. Sadullaev gives in [93] examples of entire mappings $\mathbf{C}^{2} \rightarrow \mathbf{C}^{2}$ whose ranges avoid large sets. His mappings $G_{f}$ satisfy the functional equations $F_{f} \circ G_{f}=G_{f} \circ A$, where $F_{f}$ is the automorphism $(x, y) \rightarrow\left(y, a^{2} x+f(y)\right)$, where $A=\left(\begin{array}{cc}a & 0 \\ 0 & -a\end{array}\right)$, $|a|>1$, and where $f$ is an entire function over $\mathbf{C}$ satisfying $f(0)=f^{\prime}(0)=0$. Here $A$ is the diagonal form of $F_{f}^{\prime}(0)=\left(\begin{array}{cc}0 & 1 \\ a^{2} & 0\end{array}\right)$. Since $F_{f}^{2}$ is the map $(x, y) \rightarrow$ $\left(a^{2} x+f(y), a^{2} y+f\left(a^{2} x+f(y)\right)\right)$ and since $\left(F_{f}^{2}\right)^{\prime}(0)=\left(\begin{array}{ll}0 & 1 \\ a^{2} & 0\end{array}\right)\left(\begin{array}{cc}0 & 1 \\ a^{2} & 0\end{array}\right)=a^{2} I$, 


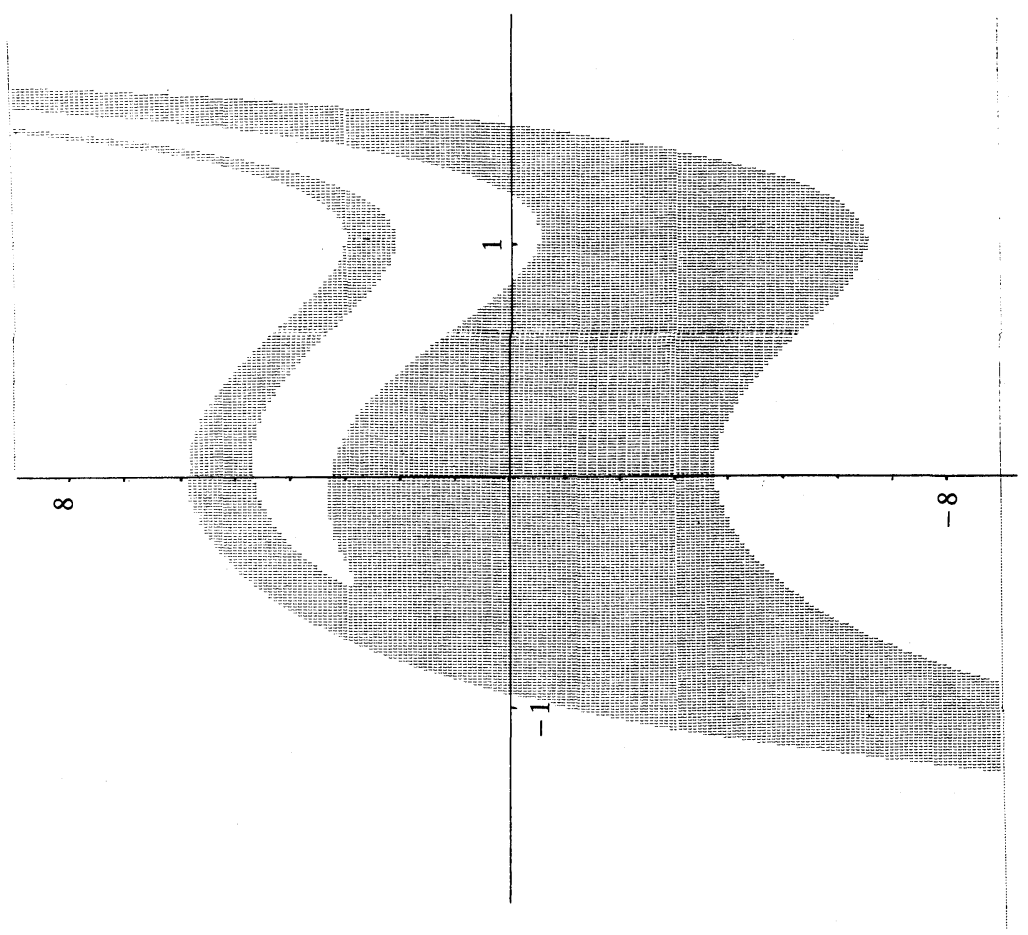

$N$

ญี

$\stackrel{1}{\Xi}$

กิ

छ.

$\| \cdot 5$

$\circ$ 응

11 은

N2

हี

売莺

ธิ

冚

袍

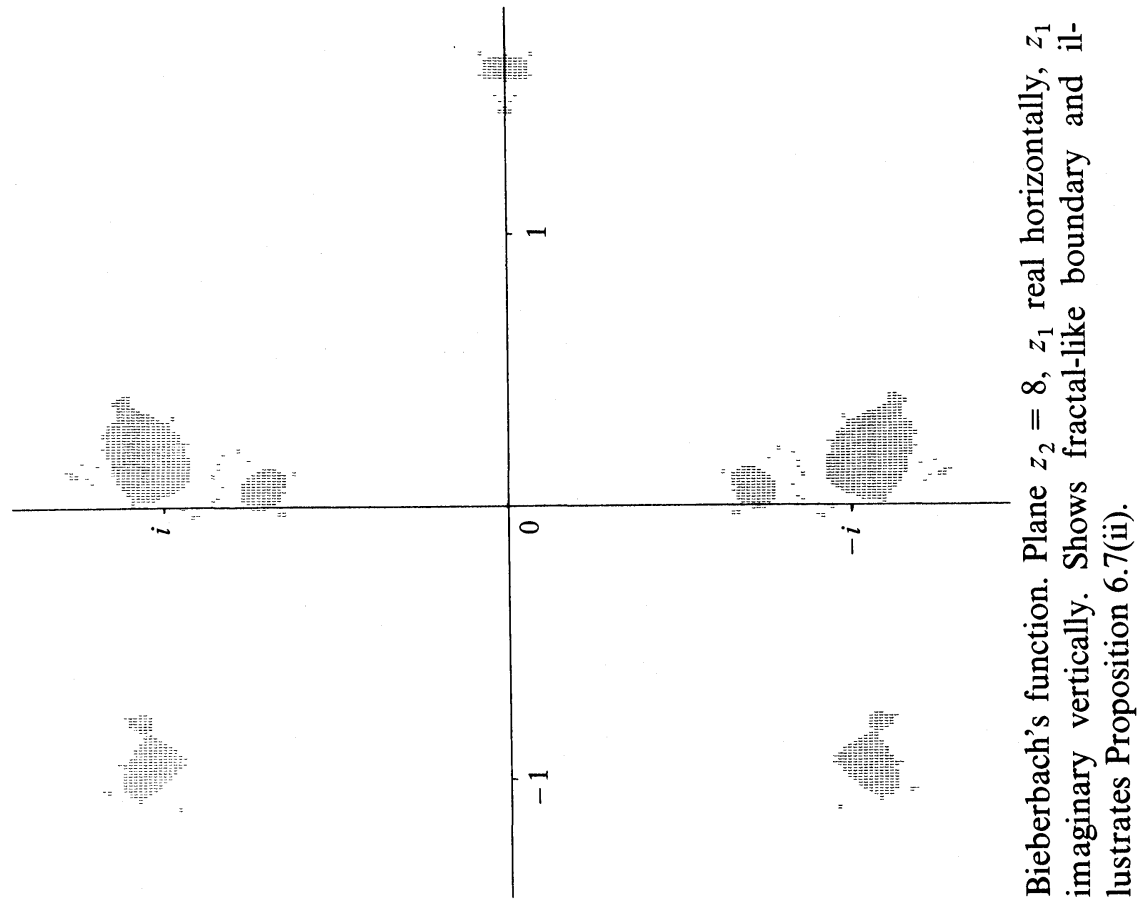


Sadullaev functions, which satisfy as well the functional equation $F_{f}^{2} \circ G_{f}=$ $G_{f} \circ A^{2}$, are more related to Bieberbach's original example than to Fatou's, and the above maps $G_{k, j}$ are of the same type as Sadullaev's, with $k=a^{2}, j=f$. When $f$ is a monomial of degree $\geqslant 2$, Sadullaev obtains estimates which show that his functions $G_{f}$ have a range which avoids some large sets. Sadullaev's functions are one-to-one and are uniform limits on compact sets of automorphisms with constant jacobian (the constant is not one, but this can be changed by a trivial normalization).

The two one-to-one entire functions $G_{1}$ and $G_{2}$ which appeared in the above description of Bieberbach's construction satisfy $G_{1}\left(\mathbf{C}^{2}\right) \cap G_{2}\left(\mathbf{C}^{2}\right)=\varnothing$. Now set $H_{n}=G_{1}^{n} \circ G_{2}(n \geqslant 1)$. Since $G_{1}$ and $G_{2}$ are one-to-one, the functions $H_{n}$ are one-to-one and $H_{n}\left(\mathbf{C}^{2}\right) \cap H_{m}\left(\mathbf{C}^{2}\right)=\varnothing$ for $n, m \geqslant 1, n \neq m$. Another way to obtain an infinite sequence of one-to-one entire functions with disjoint ranges was communicated to the authors by W. Rudin [91]; we present a special case of his construction. Let $\left(\alpha_{n}\right)$ be a discrete sequence of complex numbers. It follows from standard interpolation results [27, exercise 5, p. 209] that there exists an entire function $f$ over $\mathbf{C}$ such that $f\left(-\frac{2}{3} \alpha_{n}\right)=\alpha_{n}$, $f^{\prime}\left(-\frac{2}{3} \alpha_{n}\right)=0(n \geqslant 1)$. Consider the map $F:(x, y) \rightarrow(2 x+f(x+y),-2 y+$ $f(x+y))$. Then $F \in \operatorname{Aut}\left(\mathbf{C}^{2}\right)$, and $J(F) \equiv-4$.

Also $F\left(-\alpha_{n}, \alpha_{n} / 3\right)=\left(-\alpha_{n}, \alpha_{n} / 3\right), \quad F^{\prime}\left(-\alpha_{n}, \alpha_{n} / 3\right)=\left(\begin{array}{cc}2 & 0 \\ 0 & -2\end{array}\right)(n \geqslant 1)$. It follows then from Corollary 6.6 that there exists a sequence $\left(G_{n}\right)_{n \geqslant 1}$ of one-to-one mappings $\mathbf{C}^{2} \rightarrow \mathbf{C}^{2}$ such that

$$
G_{n}\left(\mathbf{C}^{2}\right)=\left\{z \in \mathbf{C}^{2} \mid F^{-r}(z) \underset{r \rightarrow \infty}{\rightarrow}\left(-\alpha_{n}, \alpha_{n} / 3\right)\right\}
$$

so that $G_{n}\left(\mathbf{C}^{2}\right) \cap G_{m}\left(\mathbf{C}^{2}\right)=\varnothing$ for $n \neq m$.

Another interesting method for producing an infinite sequence of one-to-one entire functions from $\mathbf{C}^{2}$ into itself with disjoint ranges is given by Kodaira in [61]. Kodaira considers the automorphism $F$ of $\mathbf{C}^{2}$ given by the formula

$$
F(x, y)=(a y+(a-1)(\sin x-x), x)
$$

where $|a|>1$.

Then $(2 k \pi, 2 k \pi)$ is a repulsive fixed point for $F$ for every $k \in \mathbf{Z}$, and $F^{\prime}(2 k \pi, 2 k \pi)=\left(\begin{array}{ll}0 & a \\ 1 & 0\end{array}\right)$. Denote by $\tau$ the map $(x, y) \rightarrow(x+2 \pi, y+2 \pi)$. Then $F \circ \tau=\tau \circ F$. Kodaira's function $G: \mathbf{C}^{2} \rightarrow \mathbf{C}^{2}$ satisfies the functional equation $F \circ G=G \circ F^{\prime}(0), \quad G(0)=0, \quad G^{\prime}(0)=I \quad$ and $\quad G\left(\mathbf{C}^{2}\right)=\{z \in$ $\left.\mathbf{C}^{2} \mid F^{-n}(z) \underset{n \rightarrow \infty}{\rightarrow} 0\right\}$. For each $k \in \mathbf{Z}$, we have

$$
\begin{aligned}
\left(\tau^{k} \circ G\right)\left(\mathbf{C}^{2}\right) & =\left\{z \in \mathbf{C}^{2} \mid\left(F^{-n} \circ \tau^{-k}\right)(z) \underset{n \rightarrow \infty}{\rightarrow} 0\right\} \\
& =\left\{z \in \mathbf{C}^{2} \mid\left(\tau^{-k} \circ F^{-n}\right)(z) \underset{n \rightarrow \infty}{\rightarrow} 0\right\} \\
& =\left\{z \in \mathbf{C}^{2} \mid F^{-n}(z) \underset{n \rightarrow \infty}{\rightarrow}(2 k \pi, 2 k \pi)\right\} .
\end{aligned}
$$

So the sequence $\left(\tau^{k} \circ G\right)_{k \in \mathbf{Z}}$ is a sequence of entire one-to-one mappings over $\mathbf{C}^{2}$ with disjoint ranges.

Another way to obtain a sequence of pairwise disjoint open subsets of $\mathbf{C}^{2}$ biholomorphically equivalent to $\mathbf{C}^{2}$ is to consider a map $F:(x, y) \rightarrow(u, v)$ where $u=2 x+f(y), v=2 y+g(u)$. Let $\left(\alpha_{n}\right)$ and $\left(\beta_{n}\right)$ be two discrete 
sequences of complex numbers. There exist two entire functions $f$ and $g$ over $\mathrm{C}$ such that $f\left(\beta_{n}\right)=-\alpha_{n}, f^{\prime}\left(\beta_{n}\right)=0, g\left(\alpha_{n}\right)=-\beta_{n}, g^{\prime}\left(\alpha_{n}\right)=0(n \geqslant 1)$. Then $F\left(\alpha_{n}, \beta_{n}\right)=\left(\alpha_{n}, \beta_{n}\right), F^{\prime}\left(\alpha_{n}, \beta_{n}\right)=2 I$ and it follows again from Corollary 6.6 that there exists a sequence $\left(G_{p}\right)$ of one-to-one entire mappings $\mathbf{C}^{2} \rightarrow \mathbf{C}^{2}$ such that $G_{p}\left(\mathbf{C}^{2}\right)=\left\{z \in \mathbf{C}^{2} \mid F^{-n}(z) \underset{n \rightarrow \infty}{\rightarrow}\left(\alpha_{p}, \beta_{p}\right)\right\}$, so that $G_{p}\left(\mathbf{C}^{2}\right) \cap G_{q}\left(\mathbf{C}^{2}\right)=\varnothing$ for $p \neq q$. The square of the automorphism used by Kodaira has a form similar to $F$, and a similar remark holds, via a linear change of variables, for the square of the automorphism used by Rudin. We leave the details to the reader.

The above constructions associate to each automorphism $F$ on $\mathbf{C}^{p}$ which possesses a repulsive fixed point $\alpha$ a one-to-one entire function $G$ on $\mathbf{C}^{p}$ such that $G\left(\mathbf{C}^{p}\right)=\left\{z \in \mathbf{C}^{p} \mid F^{-n}(z) \underset{n \rightarrow \infty}{\rightarrow} \alpha\right\}$. In his recent paper [76] Nishimura considers analytic automorphisms $F$ of $\mathbf{C}^{p}$ (in fact automorphisms of complex analytic manifolds) which possess a subvariety $Y$ of $\mathbf{C}^{p}$ which is pointwise fixed by $F$ and is repulsive for $F$ (Nishimura works in the "attractive" case but his results trivially transfer to the "repulsive" case). He then solves a functional equation similar to the equation $F \circ G=G \circ B$, under some additional hypotheses, where $B$ is the automorphism of the fiber normal to $Y$ induced by $F$. This allows him to obtain an entire one-to-one function $G$ on $\mathbf{C}^{p}$ whose range contains a neighborhood of $Y$ (namely, the elements of $\mathbf{C}^{p}$ whose sequences of iterates through $F^{-1}$ converge to elements of $Y$ ). If, further, $F$ possesses a repulsive fixed point $\alpha$ outside $Y$, the range of $G$ avoids a neighborhood of $\alpha$ and applying Corollary 6.2 to $F$ and $\alpha$ we obtain a one-to-one entire function $H$ on $\mathrm{C}^{p}$ whose range avoids a neighborhood of $Y$. D. Couty gave in [28] computations about automorphisms of $\mathbf{C}^{2}$ satisfying these two conditions. This leads, for example, to the existence of an entire one-to-one function on $\mathbf{C}^{2}$ whose range avoids the set $\left\{(u, v) \in \mathbf{C}^{2}|| u v|<1| v \mid,<1 / 2\right\}$.

We took Reich's results [88] as the key to all the results of the beginning of this section. In fact the functional equation $F \circ G=G \circ B$, where $F$ is a local automorphism with fixed point at $\alpha$ for some $\alpha \in \mathbf{C}^{p}$ and $B$ is a "normal form" for $F$, has a very long history. Assume for convenience that $\alpha=$ $(0, \ldots, 0)$, denoting this by 0 , and let $\lambda_{1}, \ldots, \lambda_{p}$ be the eigenvalues of $F^{\prime}(0)$ with $\left|\lambda_{1}\right| \leqslant \cdots \leqslant\left|\lambda_{p}\right|$. For $q \geqslant 1$ denote by $\pi_{q}(F)$ the Taylor expansion of $F$ at 0 taken up to the $q$ th order. If $\left|\lambda_{1}\right|>1,\left|\lambda_{1}\right|^{q+1}>\left|\lambda_{p}\right|$ then the sequence $\left(F^{n} \circ\left(\pi_{q}(F)\right)^{-n}\right)_{n \geqslant 1}$ converges uniformly on some neighborhood of the origin to the germ of an analytic automorphism $G$, which satisfies $G(0)=0, G^{\prime}(0)=I$, $F \circ G=G \circ \pi_{q}(F)$. If $\left|\lambda_{1}\right|^{2}>\left|\lambda_{p}\right|$ we can take $q=1$ and $\pi_{q}(F)=F^{\prime}(0)$. In this case, if $F$ is entire, the sequence $\left(F^{n} \circ\left(F^{\prime}(0)\right)^{-n}\right)$ converges uniformly on compact subsets of $\mathrm{C}^{p}$ to an entire function $G$ satisfying $F \circ G=G \circ F^{\prime}(0)$, $G(0)=0, G^{\prime}(0)=I$ (Corollary 6.6). In Bieberbach's construction we have $J(F) \equiv 16=\operatorname{det} F^{\prime}(0)$, so that the automorphism $F^{n} \circ\left[F^{\prime}(0)\right]^{-n}$ has jacobian 1 for every $n \geqslant 1$ and Bieberbach's function is a one-to-one function of jacobian 1. Kodaira and Sadullaev's functions can also be obtained this way (via an obvious renormalization in Sadullaev's case). This way of solving the equation $F \circ G=G \circ F^{\prime}(0)$ was rediscovered by the authors, and, independently, by W. Rudin, but this idea is not new at all. M. Koenigs used it in 1882 
in his beautiful thesis [62] to solve Schröder's equation $f[g(z)]=g(a z)$, where $f(0)=0, f^{\prime}(0)=a$, when $|a|>1$, and Schröder's equation is exactly the equation $F \circ G=G \circ F^{\prime}(0)$ in dimension 1 (Schröder's equation, introduced by Schröder in $[95,96]$ is related to the well-known Abel's equation introduced by Abel in [1]). This method was used by Sternberg in 1958 [102] to solve the functional equation $F \circ G=G \circ \pi_{q}(F)$ (under the conditions $\left|\lambda_{1}\right|>1,\left|\lambda_{1}\right|^{q+1}$ $\left.>\left|\lambda_{p}\right|\right)$, ten years before Reich's papers. Poincaré studied in his 1890 paper [85] the functional equation $F \circ G=G \circ F^{\prime}(0)$ in the case where $F$ is a rational map on $\mathbf{C}^{p}$ with rational inverse such that $F(0)=0, F^{\prime}(0)=k I,|k|>1$. His method, "méthode des séries majorantes", gives in fact a local solution $G$ if $F$ is any local automorphism at 0 (in $p$ complex variables) satisfying $F(0)=0$, $F^{\prime}(0)=k I,|k|>1$ (as mentioned above, this construction led Poincaré to discover in the same paper the existence of nondegenerate entire functions on $\mathbf{C}^{p}$ with nondense range). Calculations similar to Poincaré's were repeated by Bieberbach [11] and Stehlé [101] to solve the same functional equations.

E. Picard studied some related functional equations in several papers $[80,81$, 82,83 ]. In the first two he was interested in obtaining functions satisfying some "addition properties" with respect to a birational map $\mathbf{C}^{p} \rightarrow \mathbf{C}^{p}$, which are the $p$-dimensional analogues of the solutions of Abel's equation.

In $[82,83]$ he studied the functional equation $F \circ G=G \circ F^{\prime}(0)$ when $F^{\prime}(0)$ is diagonalizable and when 0 is a repulsive fixed point for $F$. Unfortunately, his 1904 paper is not entirely correct. The subtle successive approximation method described in [83] works only if the eigenvalues $\lambda_{1}, \ldots, \lambda_{p}$ of $F^{\prime}(0)$ satisfy $\lambda_{i} \neq \lambda_{1}^{n_{1}} \cdots \lambda_{p}^{n_{p}}$ for $1 \leqslant i \leqslant p, n_{1}, \ldots, n_{p} \geqslant 0, n_{1}+\cdots+n_{p} \geqslant 2$. In fact, if the above condition is not satisfied, i.e., if there is a "relation" between the eigenvalues, then no solution of the functional equation $F \circ G=G \circ F^{\prime}(0)$ exists in general, even if we look only for "formal solutions" $G$, i.e., "solutions" given by series which might be divergent. This case was first treated by Leau in his magnificent 1897 thesis [67]. In the case where relations between $\lambda_{1}, \ldots, \lambda_{p}$ do exist (with $1<\left|\lambda_{1}\right| \leqslant \cdots \leqslant\left|\lambda_{p}\right|$ ), he gets a formal solution of the functional equation $F \circ G=G \circ B$, where $B$ is a polynomial automorphism of $\mathrm{C}^{p}$ which differs from Reich's normal form only by the fact that the linear part of $B$ is given in a triangular form instead of in the Jordan form given by Reich [88]. Under a technical hypothesis he shows also that his formal solutions are actually the germs of analytic maps at the origin. Lattès gave in 1911 a complete solution when $p=2$ [65] (but the most difficult case is already contained in Leau's paper). Sternberg's 1957 paper [102] solves the general case, giving a normal form similar to Leau's, which is slightly less precise than Reich's.

If 0 is not repulsive for $F$, it is still possible to give a "formal normal form" which reduces to $F^{\prime}(0)$ when no relations between $\lambda_{1}, \ldots, \lambda_{p}$ occur. In fact $F$ can be uniquely written in the form $F=S \circ T=T \circ S$, where $S$ is "formally diagonalizable" and $T$ has a unipotent linear part. This fact can be deduced from the Jordan-Chevalley decomposition [57, Theorem 15.3] in algebraic groups, since the group $g$ of formal maps $F$ satisfying $F(0)=0, J(F)(0) \neq 0$ can be written as a projective limit of algebraic groups. The normal form, which might not be a polynomial when infinitely many relations occur, is then 
obtained by writing the diagonalizable factor $S$ in a diagonal form $S_{1}$, by a formal change of variables. The corresponding form $T_{1}$ of $T$ has to commute with the diagonal map $S_{1}$. This means that $T_{1}$ is linear if no relations between the eigenvalues of $F^{\prime}(0)$ occur, and that $T_{1}$ is a polynomial if there are only finitely many relations, which is always the case if $1<\left|\lambda_{1}\right| \leqslant \cdots \leqslant\left|\lambda_{p}\right|$. We thus obtain a formal normal form $B=S_{1} \circ T_{1}$ for $F$, and a formal mapping $G$ satisfying $F \circ G=G \circ B$. If $F$ is the germ of an analytic automorphism with repulsive fixed point then it is possible to show that the series defining $G$ actually converge over some neighborhood of the origin in $\mathbf{C}^{p}$. Siegel showed in [99] that there is also, when $p=1$, a germ $G$ of an analytic automorphism at the origin satisfying $F \circ G=G \circ F^{\prime}(0)$ if $\left|F^{\prime}(0)\right|=1$ and if $F^{\prime}(0)$ satisfies some diophantine condition, and a similar result holds in dimension $p$ if the eigenvalues $\lambda_{1}, \ldots, \lambda_{p}$ of $F^{\prime}(0)$ satisfy $\left|\lambda_{i}-\lambda_{1}^{n_{1}} \cdots \lambda_{p}^{n_{p}}\right|>\left(n_{1}+\cdots+n_{p}\right)^{-\nu}$ $\left(1 \leqslant i \leqslant p, n_{1} \geqslant 0, \ldots, n_{p} \geqslant 0, n_{1}+\cdots+n_{p} \geqslant 2\right)$ for some $\nu>0$, see [92, $103,113]$ or $[9$, Chapter V]. We will not give further historical comments here, and we refer to a forthcoming expository paper [25] by B. Chevreau and the second author for a detailed presentation of the formal theory of normal forms via the Jordan-Chevalley theorem in algebraic groups and for further details about Picard's approximation method, a very powerful one despite the error in some statements of [83].

In general there is no germ $G$ of an analytic automorphism at the origin satisfying $F \circ G=G \circ B$, where $B$ is the "formal normal form" described above, even if $B$ is a polynomial or a linear map. There was recent interest in the theory of topological and analytical classification of such maps $F$, see Camacho [19], Martinet and Ramis [71], Sherbakov [97], and Voronin [107] in dimension one. See also Belitskii [12] and Russman [92] for higher dimensions. There is also a rich literature concerning real variable analogues of the functional equations discussed above, see Kuczma's book [63].

The theory of normal forms for germs of analytic automorphisms has a counterpart, the theory of normalization of differential equations (or the theory of normalization of holomorphic flows). The idea there is, by a suitable analytic change of variables, to turn some differential equations which present a singularity at the origin into linear, or at least into simpler differential equations. The seminal paper in this area is the thesis of Poincare [84], and the counterparts of the "relations" described above are called "resonances". The connections between the two theories can be interpreted through an exponential map between the "infinite Lie group" $g$ introduced above and its "infinite Lie algebra", see Sternberg [102, 103], Arnold [9, Chapter V] and also Martinet and Ramis [71]. Poincaré's hypotheses exclude "resonances", which were first studied by Dulac [37]; there is also some recent interest in this area, see Bruno [17], Camacho [19], Camacho and Sad [20], Ecalle [40], Elizarov and Ilyashenko [41], Françoise [47], Malgrange and Martinet's talks at the Bourbaki seminar $[68,70]$ and Martinet and Ramis [71, 72], where further references can be found (see also Dulac [38]).

The range of Bieberbach's original function is the set

$$
\left\{z \in \mathbf{C}^{2} \mid F^{-n}(z) \underset{n \rightarrow \infty}{\rightarrow} 0\right\}
$$


where $F$ is a polynomial automorphism. The theory of iteration of polynomial and rational maps is very complicated, even in dimension 1 . The basic work in dimension 1 is due to Julia [58, 59] and Lattès obtained some results in dimension 2 in [66]. There is also a recent renewal of interest in this area, see for example Mane, Sad and Sullivan [69], Sullivan and Thurston [105] and Douady's talk at the Bourbaki seminar [35] where further references can be found.

Denote by $\mathrm{Aut}_{1}\left(\mathbf{C}^{p}\right)$ the set of all analytic automorphisms of $\mathbf{C}^{p}$ of jacobian 1. One heuristic consequence of the above discussion is the fact that Bieberbach's original map is the uniform limit on compact sets of a sequence of analytic automorphisms of jacobian 1 . This leads to the following definition.

Definition 6.8. A Bieberbach function is an analytic map $G: \mathbf{C}^{p} \rightarrow \mathbf{C}^{p}$, with nondense range, such that $\Theta_{n} \underset{n \rightarrow \infty}{\stackrel{\sigma}{\rightarrow}} G$ for some sequence $\left(\Theta_{n}\right)$ of elements of Aut $_{1}\left(\mathbf{C}^{p}\right)$. The set of Bieberbach functions is denoted by $B\left(C^{p}\right)$.

It follows from Corollary 6.6 that the original example of Bieberbach [11], Kodaira's function [61] and, up to a renormalization, Sadullaev's functions [93] satisfy the above conditions.

It follows from Corollary 5.3 that Bieberbach functions are one-to-one, have jacobian one and that their range is a Runge domain. Moreover the range of such a function $G$ is the set of all $z \in \mathbf{C}^{p}$ such that $\left|\Theta_{n}^{-1}(z)\right|_{n \rightarrow \infty}^{\rightarrow \infty} \infty$. These observations will lead to a new method for constructing such functions, to be developed in $\S 8$.

7. Iteration of entire mappings. We will first show in this section that for every sequence $\left(F_{n}\right)$ of nondegenerate elements of $\mathscr{H}\left(\mathbf{C}^{p}, \mathbf{C}^{p}\right)$ there exists $G \in \mathscr{H}\left(\mathbf{C}^{p}, \mathbf{C}^{p}\right)$ such that $\left(G \circ F_{1} \circ \ldots \circ F_{n}\right)\left(\mathbf{C}^{p}\right)$ is onto for every $n \geqslant 1$ (and if $\left(\mathbf{C}^{p_{n}}, F_{n}\right)_{n \geqslant 1}$ is a projective system with $F_{n}: \mathbf{C}^{p_{n+1}} \rightarrow \mathbf{C}^{p_{n}}$ entire and $F_{1} \circ \cdots \circ F_{n}$ not constant there exists $G \in \mathscr{H}\left(\mathbf{C}^{p_{1}}, \mathbf{C}^{p_{1}}\right)$ such that $\bigcap_{n \geqslant 1}\left(G \circ F_{1} \circ \cdots \circ F_{n}\right)\left(\mathbf{C}^{p_{n}}\right)$ is dense in $\left.\mathbf{C}^{p_{1}}\right)$. The function $G$ can be chosen arbitrarily close to the identity map with respect to the $\sigma$-topology, but this result does not indeed give any information about $\bigcap_{n \geqslant 1} F_{1} \circ \cdots \circ F_{n}\left(\mathbf{C}^{p}\right)$ since these functions $G$ are not one-to-one (they cannot be one-to-one if $F_{1}\left(\mathbf{C}^{p}\right)$ is not dense in $\mathbf{C}^{p}$ ). We obtain as a corollary the fact, proved by Gauthier and Ngô Van Quê in [49], that functions with dense range are dense in $\mathscr{H}\left(\mathbf{C}^{p}, \mathbf{C}^{q}\right)$ and that surjective functions are dense in $\mathscr{H}\left(\mathbf{C}^{p}, \mathbf{C}^{p}\right)$.

We also study the relationship between $\operatorname{Fr}\left(F_{1}\left(\mathbf{C}^{p}\right)\right)$ and $\operatorname{Fr}\left(\left(F_{1} \circ F_{2}\right)\left(\mathbf{C}^{p}\right)\right)$ when $F_{1}$ and $F_{2}$ are entire and one-to-one. If $K$ is a compact subset of $F_{1}\left(\mathbf{C}^{p}\right)$ and if $F_{1}$ is one-to-one there exists a Bieberbach function $F_{2}$ such that $F_{2}\left(\mathbf{C}^{p}\right) \cap F_{1}^{-1}(K)=\varnothing$, so that $\left(F_{1} \circ F_{2}\right)\left(\mathbf{C}^{p}\right) \cap K=\varnothing$. Using this observation it is easy to construct a sequence $\left(F_{n}\right)$ of Bieberbach functions such that the interior of $\bigcap_{n \geqslant 1}\left(F_{1} \circ \cdots \circ F_{n}\right)\left(\mathbf{C}^{p}\right)$ is empty. What we show is that it is also possible to arrange that

$$
\operatorname{Fr}\left[\left(F_{1} \circ \cdots \circ F_{n}\right)\left(\mathbf{C}^{p}\right)\right] \subset \operatorname{Fr}\left[\left(F_{1} \circ \cdots \circ F_{n+1}\right)\left(\mathbf{C}^{p}\right)\right] \quad(n \geqslant 1) .
$$

This follows from the fact that if $F_{1}$ is one-to-one and if $K$ is a compact subset of $F_{1}\left(\mathbf{C}^{p}\right)$, then there exists a Bieberbach function $F_{2}$ such that $\operatorname{Fr}\left[F_{1}\left(\mathbf{C}^{p}\right)\right] \subset$ $\operatorname{Fr}\left[\left(F_{1} \circ F_{2}\right)\left(\mathbf{C}^{p}\right)\right]$ and $\left(F_{1} \circ F_{2}\right)\left(\mathbf{C}^{p}\right) \cap K=\varnothing$. The proof involves sequences of 
elements of $\mathrm{Aut}_{1}\left(\mathbf{C}^{p}\right)$ which converge to the identity map with respect to the $\sigma$-topology but whose elements map some sequences of large elements of $\mathbf{C}^{p}$ onto some very different ones.

We conclude this section with the observation that, if $G$ is Bieberbach's original function (or one of Sadullaev's functions [93]) there exists a bounded set $B$ such that $G^{-1}(B)$ meets the range of every nonconstant entire function $H: \mathbf{C}^{2} \rightarrow \mathbf{C}^{2}$. In fact there exists for every $\varepsilon>0$ a Bieberbach function $F$ such that $F\left(\mathbf{C}^{2}\right) \cap D(0,1)=\varnothing$ and such that $(F \circ H)\left(\mathbf{C}^{2}\right) \cap D(0,1+\varepsilon) \neq \varnothing$ for every nonconstant entire function $H: \mathbf{C}^{2} \rightarrow \mathbf{C}^{2}$. So Bieberbach's original example and Sadullaev's examples are not good starts if one wants to construct a sequence $\left(F_{n}\right)$ of entire functions over $\mathbf{C}^{2}$ satisfying $\left(F_{1} \circ \ldots \circ F_{n}\right)\left(\mathbf{C}^{2}\right) \cap$ $D(0, n)=\varnothing(n \geqslant 1)$ and there exist one-to-one entire functions $F: \mathbf{C}^{2} \rightarrow \mathbf{C}^{2}$ such that

$$
\bigcap_{n \geqslant 1} \overline{\left(F \circ G_{1} \circ \cdots \circ G_{n}\right)\left(\mathbf{C}^{2}\right)} \neq \varnothing
$$

for every sequence $\left(G_{n}\right)$ of entire functions from $\mathbf{C}^{2}$ into itself. These results follow from the elementary computations of Proposition 6.7.

We begin the section with two lemmas about entire functions of one complex variable, which follow from standard interpolation results and from Runge's theorem.

LEMMA 7.1. Let $\left(\alpha_{n}\right)$ and $\left(\beta_{n}\right)$ be two sequences of complex numbers, and let $f$ be an entire function over $\mathbf{C}$. If all the $\alpha_{n}$ are distinct, and if $\liminf _{n \rightarrow \infty}\left|\alpha_{n}\right|=$ $+\infty$, then there exists a sequence $\left(f_{m}\right)$ of entire functions satisfying the following conditions.

(1) $f_{m} \underset{m \rightarrow \infty}{\stackrel{\sigma}{\rightarrow}} f$.

(2) For every $m \geqslant 1, f_{m}\left(\alpha_{n}\right)=\beta_{n}$ when $n$ is large enough.

Proof. For every $m \geqslant 1$ there exists $q_{m} \in \mathbf{N}$ such that $\alpha_{n}>m\left(n>q_{m}\right)$. It follows from standard interpolation results [27, Exercise 5, p. 209] that there exists an entire function $g$ such that $g\left(\alpha_{n}\right)=\beta_{n}(n \geqslant 1)$. Also it follows from the theory of Weierstrass products that there exists an entire function $h$ whose set of zeros is exactly the set $\left\{\alpha_{n}\right\}_{n>q_{m}}$. For $i \geqslant 1$ denote by $P_{i}$ the function

$$
z \rightarrow \sum_{j=0}^{i}\left(\frac{f-g}{h}\right)^{(j)}(0) \frac{z^{j}}{j !}
$$

Set $M=\sup _{|z| \leqslant m}|h(z)|$. Then

$$
\sup _{|z| \leqslant m}\left|f(z)-g(z)-h(z) P_{i}(z)\right| \leqslant M \sup _{|z| \leqslant m}\left|\frac{f(z)-g(z)}{h(z)}-P_{i}(z)\right| \underset{i \rightarrow \infty}{\rightarrow} 0 .
$$

Taking $f_{m}=g+h P_{i}$, with $i$ large enough, we obtain $\sup _{|z| \leqslant m}\left|f(z)-f_{m}(z)\right|$ $\leqslant 1 / m$ and $f_{m}\left(\alpha_{n}\right)=\beta_{n}\left(n>q_{m}\right)$. So the sequence $\left(f_{m}\right)_{m \geqslant 1}$ satisfies the desired conditions. 
LEMMA 7.2. Let $\left(\Omega_{n}\right)$ be a sequence of open discs in $\mathbf{C}$ with disjoint closures such that $d\left(0, \Omega_{n}\right) \underset{n \rightarrow \infty}{\rightarrow} \infty$, let $f$ be an entire function and let $\left(\beta_{n}\right)$ be a sequence of elements of $\mathbf{C}$. There exists a sequence $\left(f_{m}\right)$ of entire functions satisfying the following two conditions.

(1) $f_{m} \underset{m \rightarrow \infty}{\stackrel{\sigma}{\rightarrow}} f$.

(2) For every $m \geqslant 1, D\left(\beta_{n}, 1\right) \subset f_{m}\left(\Omega_{n}\right)$ when $n$ is large enough.

Proof. Denote by $\delta_{n}$ the radius of $\Omega_{n}$, denote by $\alpha_{n}$ the center of $\Omega_{n}$, and denote by $h_{n}$ the functions $z \rightarrow \beta_{n}+2\left(z-\alpha_{n}\right) / \delta_{n}$. We can construct a strictly increasing sequence $\left(q_{m}\right)$ of integers such that $\bar{\Omega}_{n} \cap \overline{D(0, m)}=\varnothing\left(n \geqslant q_{m}\right)$. For $m \geqslant 1$ set $K_{m}=\left(\bigcup_{n<q_{m}} \bar{\Omega}_{n}\right) \cup \overline{D(0, m)}$.

It follows from Runge's theorem that there exists a polynomial $P_{0}$ such that

$$
\left\|P_{0}-f\right\|_{K_{m}}<1 / 2 m, \quad\left\|P_{0}-h_{k}\right\|_{\bar{\Omega}_{k}}<1 / 2
$$

$\left(q_{m} \leqslant k<q_{m+1}\right)$. By a repeated use of Runge's theorem we can then construct by induction a sequence $\left(P_{i}\right)_{i \geqslant 1}$ of polynomials such that

$$
\left\|P_{i}\right\|_{K_{m+\imath}}<\frac{2^{-i-1}}{m}, \quad\left\|\sum_{j=0}^{i} P_{j}-h_{k}\right\|_{\bar{\Omega}_{k}}<2^{-i-1}
$$

$\left(q_{m+i} \leqslant k<q_{m+i+1}\right)$. It follows from the first condition that the series $\sum_{j=0}^{\infty} P_{j}$ converges uniformly over compact subsets of $\mathbf{C}$ to an entire function $f_{m}$. We have $\sup _{|z| \leqslant m}\left|f_{m}(z)-f(z)\right|<1 / m$, so that $f_{m} \underset{m \rightarrow \infty}{\stackrel{\sigma}{\rightarrow}} f$.

Also

$$
\left\|f_{m}-h_{k}\right\|_{\bar{\Omega}_{k}}<2^{-i-1}+\sum_{j=i+1}^{\infty}\left\|P_{j}\right\|_{K_{m+j}}<1
$$

if $q_{m+i} \leqslant k<q_{m+i+1}(i \geqslant 0)$. So $\sup _{\left|\xi-\alpha_{n}\right|=\delta_{n}}\left|f_{m}(\xi)-h_{n}(\xi)\right|<1$ for $n \geqslant q_{m}$. Since $\left|h_{n}(\xi)-\beta_{n}\right|=2$ we have $\left|h_{n}(\xi)-u\right| \geqslant 1$ for $\left|\xi-\alpha_{n}\right|=\delta_{n},\left|u-\beta_{n}\right|<1$. It follows then from Rouché's theorem that the equation $f_{m}(z)=u$ has a solution in $\Omega_{n}$ for $\left|u-\beta_{n}\right|<1$. So $D\left(\beta_{n}, 1\right) \subset f_{m}\left(\Omega_{n}\right)\left(m \geqslant 1, n \geqslant q_{m}\right)$ and the sequence $\left(f_{m}\right)_{m \geqslant 1}$ satisfies the conditions of the lemma.

For $i \leqslant p$ we denote by $\pi_{i}:\left(x_{1}, \ldots, x_{p}\right) \rightarrow x_{i}$ and $\pi_{(i)}:\left(x_{1}, \ldots, x_{p}\right) \rightarrow$ $\left(x_{1}, \ldots, x_{i-1}, x_{i+1}, \ldots, x_{p}\right)$ the canonical projections.

LEMMA 7.3. Let $\left(x_{n}\right)$ be a sequence of elements of $\mathrm{C}^{p}$ such that $\lim \sup _{n \rightarrow \infty}\left|x_{n}\right|=+\infty$. There exists a sequence $\left(\Theta_{m}\right)_{m \geqslant 1}$ of elements of $\operatorname{SL}_{p}(\mathbf{C})$ and a subsequence $\left(x_{n}\right)$ of the sequence $\left(x_{n}\right)$ such that $\left\|\Theta_{m}-I\right\| \rightarrow 0$ and such that $\liminf \operatorname{in}_{i \rightarrow \infty}\left|\pi_{j} \circ \Theta_{m}\left(x_{n_{t}}\right)\right|=+\infty(m \geqslant 1, j \leqslant p)$.

Proof. We may assume without loss of generality that $\left|\pi_{k}\left(x_{n}\right)\right|_{n \rightarrow \infty} \infty$ for some $k \leqslant p$. Let $S$ be a finite subset of $\{1, \ldots, p\}$ containing $k$ which is maximal with respect to the property $\lim \sup _{n \rightarrow \infty}\left(\inf _{j \in S}\left|\pi_{j}\left(x_{n}\right)\right|\right)=+\infty$. There exists a subsequence $\left(x_{n_{t}}\right)$ of the sequence $\left(x_{n}\right)$ such that $\left|\pi_{j}\left(x_{n_{i}}\right)\right|_{i \rightarrow \infty} \infty$ for $j \in S$. For every $j \notin S$ we have $\lim \sup _{i \rightarrow \infty}\left|\pi_{j}\left(x_{n_{i}}\right)\right|<+\infty$. Define $\Theta_{m}$ for $m \geqslant 1$ by the condition $\pi_{j} \circ \Theta_{m}=\pi_{j}(j \in S), \pi_{j} \circ \Theta_{m}=\pi_{j}+\pi_{k} / m(j \notin S)$. 
Then Det $\Theta_{m}=1,\left\|\Theta_{m}-I\right\| \underset{m \rightarrow \infty}{\rightarrow} 0$ and $\left|\left(\pi_{j} \circ \Theta_{m}\right)\left(x_{n_{i}}\right)\right|_{i \rightarrow \infty}^{\rightarrow} \infty$ for every $j \leqslant p$. This proves the lemma.

Proposition 7.4. (i) For every sequence $\left(x_{n}\right)$ of elements of $\mathbf{C}^{p}$ such that $\left|x_{n}\right|_{n \rightarrow \infty} \infty$ and for every countable subset $S$ of $\mathbf{C}^{p}$ there exists a sequence $\left(\varphi_{m}\right)_{m \geqslant 1}$ of entire functions from $\mathbf{C}^{p}$ into itself such that $\varphi_{m} \underset{m \rightarrow \infty}{\stackrel{\sigma}{\rightarrow}} I$ and such that $S \subset \varphi_{m}\left[\left\{x_{i}\right\}_{i \geqslant n}\right](n \geqslant 1, m \geqslant 1)$.

(ii) For every sequence $\left(D\left(x_{n}, \delta_{n}\right)\right)$ of open polydiscs in $\mathbf{C}^{p}$ with $\left|x_{n}\right|_{n \rightarrow \infty} \infty$ there exists a sequence $\left(\Psi_{m}\right)_{m \geqslant 1}$ of entire functions from $\mathbf{C}^{p}$ into itself such that $\Psi_{m} \underset{m \rightarrow \infty}{\stackrel{\sigma}{\rightarrow}}$ I and such that

$$
\Psi_{m}\left(\bigcup_{i \geqslant n} D\left(x_{i}, \delta_{i}\right)\right)=\mathbf{C}^{p} \quad(n \geqslant 1, m \geqslant 1) .
$$

Proof. Fix $m \geqslant 1$. In both cases it follows from Lemma 7.3 that there exists $\Theta_{m} \in \mathrm{SL}_{p}(\mathbf{C})$ such that $\sup _{|z| \leqslant m}\left|\Theta_{m}(z)-z\right|<1 / m$ and such that $\left|\left(\pi_{j} \circ \Theta_{m}\right)\left(x_{n_{i}}\right)\right| \underset{i \rightarrow \infty}{\rightarrow} \infty(j \leqslant p)$ for some subsequence $\left(x_{n_{t}}\right)$ of the sequence $\left(x_{n}\right)$, and we may assume without loss of generality that all the terms of the sequence $\left(\left(\pi_{j} \circ \Theta_{m}\right)\left(x_{n_{t}}\right)\right)_{i \geqslant 1}$ are distinct for $j \leqslant p$.

To prove (i) now consider a sequence $\left(\beta_{n}\right)$ of elements of $\mathbf{C}^{p}$ such that $\beta_{n}=s$ for infinitely many values of $n$ when $s \in S$.

It follows from Lemma 7.1 that there exist entire functions $f_{1, m}, \ldots, f_{p, m}$ over $\mathbf{C}$ such that $\left|f_{j, m}(z)-z\right|<1 / m \quad(j \leqslant p,|z| \leqslant m)$ and such that $\left(f_{j, m} \circ \pi_{j} \circ \Theta_{m}\right)\left(x_{n_{i}}\right)=\pi_{j}\left(\beta_{i}\right)$ when $i$ is large enough $(j=1, \ldots, p)$. Set $F_{m}=$ $\left(f_{1, m}, \ldots, f_{p, m}\right)$ and $\varphi_{m}=F_{m} \circ \Theta_{m}$. Then $F_{m} \underset{m \rightarrow \infty}{\stackrel{\sigma}{\rightarrow}} I$ so that $\varphi_{m} \underset{m \rightarrow \infty}{\stackrel{\sigma}{\rightarrow}} I$ and $\varphi_{m}\left(x_{n_{t}}\right)=\beta_{i}$ when $i$ is large enough. So $S \subset \varphi_{m}\left[\left\{x_{i}\right\}_{i \geqslant n}\right]$ for $m \geqslant 1, n \geqslant 1$. This proves the first assertion.

To prove (ii), consider a dense sequence $\left(\beta_{n}\right)_{n \geqslant 1}$ in $\mathbf{C}^{p}$. There exists a sequence $\left(\mu_{i}\right)_{i \geqslant 1}$ of positive real numbers such that the polydisc $D\left(\Theta_{m}\left(x_{n_{t}}\right), \mu_{i}\right)$ is contained in $\Theta_{m}\left(D\left(x_{n}, \delta_{i}\right)\right)(i \geqslant 1)$ and such that the closures of the $\operatorname{discs}\left(D\left(\left(\pi_{j} \circ \Theta_{m}\right)\left(x_{n_{1}}\right), \mu_{i}\right)\right)_{i \geqslant 1}$ are disjoint for $j \leqslant p$. It follows then from Lemma 7.2 that there exist entire functions $g_{1, m}, \ldots, g_{p, m}$ over $\mathbf{C}$ such that $\left|g_{j, m}(z)-z\right|<1 / m(|z| \leqslant m, j \leqslant p)$ and such that

$$
D\left(\pi_{j}\left(\beta_{i}\right), 1\right) \subset g_{j, m}\left[D\left(\left(\pi_{j} \circ \Theta_{m}\right)\left(x_{n_{t}}\right), \mu_{i}\right)\right]
$$

when $i$ is large enough $(j \leqslant p)$. Set $G_{m}=\left(g_{1, m}, \ldots, g_{p, m}\right)$ and $\Psi_{m}=G_{m} \circ \Theta_{m}$. Then $G_{m} \underset{m \rightarrow \infty}{\stackrel{\sigma}{\rightarrow}} I$, so that $\Psi_{m} \underset{m \rightarrow \infty}{\stackrel{\sigma}{\rightarrow}} I$. Also for $m \geqslant 1$ we have $D\left(\beta_{i}, 1\right) \subset$ $\Psi_{m}\left(D\left(x_{n}, \delta_{i}\right)\right)$ when $i$ is large enough, so that $\mathrm{C}^{p} \subset \Psi_{m}\left(\bigcup_{i \geqslant n} D\left(x_{i}, \delta_{i}\right)\right)$ $(n \geqslant 1, m \geqslant 1)$. This proves the second assertion.

COROllaRY 7.5. (i) Let $\left(\mathbf{C}^{p_{n}}, F_{n}\right)$ be a projective system where $F_{n}$ : $\mathbf{C}^{p_{n+1}} \rightarrow$ $\mathrm{C}^{p_{n}}$ is entire and where $F_{1} \circ \cdots \circ F_{n}$ is not constant for $n \geqslant 1$. Then there exists a sequence $\left(\varphi_{m}\right)_{m \geqslant 1}$ of elements of $\mathscr{H}\left(\mathbf{C}^{p_{1}}, \mathbf{C}^{p_{1}}\right)$ such that

$$
\bigcap_{n \geqslant 1}\left(\varphi_{m} \circ F_{1} \circ \cdots \circ F_{n}\right)\left(\mathbf{C}^{p_{n+1}}\right)
$$

is dense in $\mathbf{C}^{p_{1}}$ for $m \geqslant 1$ and such that $\varphi_{m} \underset{m \rightarrow \infty}{\stackrel{\sigma}{\rightarrow}} I$. 
(ii) Let $p \geqslant 1$ and let $\left(F_{n}\right)$ be a sequence of nondegenerate entire functions from $\mathbf{C}^{p}$ into itself. Then there exists a sequence $\left(\Psi_{m}\right)_{m \geqslant 1}$ in $\mathscr{H}\left(\mathbf{C}^{p}, \mathbf{C}^{p}\right)$ such that $\Psi_{m} \underset{m \rightarrow \infty}{\stackrel{\sigma}{\rightarrow}} I$ and such that $\left(\Psi_{m} \circ F_{1} \circ \ldots \circ F_{n}\right)\left(\mathbf{C}^{p}\right)=\mathbf{C}^{p}(n \geqslant 1, m \geqslant 1)$.

Proof. (i) Since $\left(F_{1} \circ \cdots \circ F_{n}\right)\left(\mathbf{C}^{p_{n+1}}\right)$ is unbounded for $n \geqslant 1$, we can find a sequence $\left(x_{n}\right)_{n \geqslant 1}$ of elements of $C^{p_{1}}$ such that $\left|x_{n}\right|_{n \rightarrow \infty} \infty$ and such that $x_{n} \in\left(F_{1} \circ \cdots \circ F_{n}\right)\left(\mathbf{C}^{p_{n+1}}\right) \quad(n \geqslant 1)$. The first assertion then follows immediately from Theorem 7.4 (i), when considering a countable set $S \subset \mathbf{C}^{p_{1}}$ which is dense in $\mathbf{C}^{p_{1}}$.

(ii) Since $F_{1} \circ \cdots \circ F_{n}$ is nondegenerate, the set

$$
\left\{z \in \mathbf{C}^{p} \mid J\left(F_{1} \circ \cdots \circ F_{n}\right)(z) \neq 0\right\}
$$

is dense in $\mathbf{C}^{p}(n \geqslant 1)$. So the interior of $\left(F_{1} \circ \ldots \circ F_{n}\right)\left(\mathbf{C}^{p}\right)$ is unbounded for every $n \geqslant 1$, and we can find a sequence $\left(D\left(x_{n}, \delta_{n}\right)\right)_{n \geqslant 1}$ of open polydiscs in $\mathbf{C}^{p}$ such that $\left|x_{n}\right|_{n \rightarrow \infty} \infty$ and such that $D\left(x_{n}, \delta_{n}\right) \subset\left(F_{1} \circ \cdots \circ F_{n}\right)\left(\mathbf{C}^{p}\right)(n \geqslant$ 1). The second assertion then follows immediately from Theorem 7.4(ii).

Corollary 7.6 (Gauthier - NGÔ Van Quê [49]).

(i) Functions with dense range are dense in $\mathscr{H}\left(\mathbf{C}^{q}, \mathbf{C}^{p}\right)(p \geqslant 1, q \geqslant 1)$.

(ii) Surjective functions are dense in $\mathscr{H}\left(\mathbf{C}^{p}, \mathbf{C}^{p}\right)(p \geqslant 1)$.

Proof. To prove (i), set $p_{1}=p, p_{n}=q(n \geqslant 2), F_{1}=F, F_{n}=I(n \geqslant 2)$. If $F$ is not constant, there exists a sequence $\left(\varphi_{m}\right)_{m \geqslant 1}$ in $\mathscr{H}\left(\mathbf{C}^{p}, \mathbf{C}^{p}\right)$ such that $\varphi_{m} \underset{m \rightarrow \infty}{\stackrel{\sigma}{\rightarrow}} I$, and such that $\left(\varphi_{m} \circ F\right)\left(\mathbf{C}^{q}\right)$ is dense in $\mathbf{C}^{p}(m \geqslant 1)$. Since $\varphi_{m} \circ F \underset{m \rightarrow \infty}{\stackrel{\sigma}{\rightarrow}} F$, this proves the first assertion, because nonconstant functions are trivially dense in $\mathscr{H}\left(\mathbf{C}^{p}, \mathbf{C}^{q}\right)$.

To prove (ii), first consider a nondegenerate element $F$ of $\mathscr{H}\left(\mathbf{C}^{p}, \mathbf{C}^{p}\right)$. Taking $F_{1}=F, F_{n}=I(n \geqslant 2)$, we see that there exists a sequence $\Psi_{m}$ of elements of $\mathscr{H}\left(\mathbf{C}^{p}, \mathbf{C}^{p}\right)$ such that $\Psi_{m} \circ F$ is onto and such that $\Psi_{m} \underset{m \rightarrow \infty}{\stackrel{\sigma}{\rightarrow}} I$. So $\Psi_{m} \circ F \underset{m \rightarrow \infty}{\stackrel{\sigma}{\rightarrow}} F$, and $F$ can be approximated by surjective elements of $\mathscr{H}\left(\mathbf{C}^{p}, \mathbf{C}^{p}\right)$ with respect to the $\boldsymbol{\sigma}$-topology.

Also, by using triangulation, it is immediate to check that $\mathrm{GL}\left(\mathbf{C}^{p}\right)$ is dense in $\operatorname{End}\left(\mathbf{C}^{p}\right)$. Consider a degenerate function $F \in \mathscr{H}\left(\mathbf{C}^{p}, \mathbf{C}^{p}\right)$. There exists a sequence $\left(U_{m}\right)$ of elements of $\operatorname{End}\left(\mathbf{C}^{p}\right)$ such that $\left\|U_{m}\right\|_{m \rightarrow \infty} 0$ and such that $J\left(F+U_{m}\right)(0) \neq 0(m \geqslant 1)$. So $F+U_{m} \underset{m \rightarrow \infty}{\stackrel{\sigma}{\rightarrow}} F$ and $F+U_{m}$ is nondegenerate for every $m \geqslant 1$. This concludes the proof of the second assertion.

We now turn to the study of $\operatorname{Fr}\left(F\left(\mathbf{C}^{p}\right)\right)$ when $F: \mathbf{C}^{p} \rightarrow \mathbf{C}^{p}$ is a one-to-one entire function. Since $F\left(\mathbf{C}^{p}\right)$ is open we have $\liminf _{n \rightarrow \infty}\left|x_{n}\right|=+\infty$ if $\alpha=$ $\lim _{n \rightarrow \infty} F\left(x_{n}\right)$ where $\alpha \in \operatorname{Fr}\left(F\left(\mathbf{C}^{p}\right)\right)$. Also, since $\mathbf{C}^{p}$ is separable, $\operatorname{Fr}\left(F\left(\mathbf{C}^{p}\right)\right)$ is separable and there exists an unbounded countable set $S$ such that $\operatorname{Fr}\left(F\left(\mathbf{C}^{p}\right)\right) \subset \overline{F(S)}$. We wish to show that there are no restrictions concerning such sets $S$. More precisely we will show that given any unbounded set $S$ there exists a one-to-one entire function $G$ with the same range as $F$ such that $\operatorname{Fr}\left(G\left(\mathbf{C}^{p}\right)\right) \subset \overline{G(S)}$. 
Lemma 7.7. Let $\left(x_{n}\right)$ be a sequence of elements of $\mathbf{C}^{p}$, with $p \geqslant 2$. Assume that for some $i \leqslant p$ all the terms of the sequence $\left(\pi_{i}\left(x_{n}\right)\right)_{n \geqslant 1}$ are distinct and that $\liminf _{n \rightarrow \infty}\left|\pi_{i}\left(x_{n}\right)\right|=+\infty$. Then for every sequence $\left(u_{n}\right)$ of elements of $\mathbf{C}^{p-1}$ there exists a sequence $\left(\Theta_{m}\right)$ of elements of $\mathrm{Aut}_{1}\left(\mathbf{C}^{p}\right)$ such that $\pi_{i} \circ \Theta_{m}=\pi_{i}$ $(m \geqslant 1), \Theta_{m} \underset{m \rightarrow \infty}{\stackrel{\sigma}{\rightarrow}} I$ and such that $\left(\pi_{(i)} \circ \Theta_{m}\right)\left(x_{n}\right)=u_{n}$ eventually for each fixed $m \geqslant 1$.

Proof. Fix $m \geqslant 1$. There exists $q_{m} \geqslant 1$ such that $\left|\pi_{i}\left(x_{n}\right)\right|>m\left(n \geqslant q_{m}\right)$. It follows from Lemma 7.1 that there exist entire functions $\left(g_{j, m}\right)_{j \leqslant p, j \neq i}$ over $\mathbf{C}$ such that $\left|g_{j, m}(z)\right|<1 / m(|z| \leqslant m)$ and $\left(g_{j, m} \circ \pi_{i}\right)\left(x_{n}\right)=\pi_{j}\left(u_{n}\right)-\pi_{j}\left(x_{n}\right)$ $\left(n \geqslant q_{m}\right)$ for $j \leqslant p, j \neq i$. Define $\Theta_{m}: \mathbf{C}^{p} \rightarrow \mathbf{C}^{p}$ by the conditions $\pi_{i} \circ \Theta_{m}=\pi_{i}$, $\pi_{j} \circ \Theta_{m}=\pi_{j}+g_{j, m} \circ \pi_{i}$. Then the sequence $\left(\Theta_{m}\right)_{m \geqslant 1}$ satisfies the desired conditions.

COROLlaRY 7.8. Let $\left(x_{n}\right)$ and $\left(y_{n}\right)$ be two sequences of elements of $\mathbf{C}^{p}$, with $p \geqslant 2$. Assume that for some $i, j \leqslant p$ all the terms of the sequences $\left(\pi_{i}\left(x_{n}\right)\right)_{n \geqslant 1}$ and $\left(\pi_{j}\left(y_{n}\right)\right)_{n \geqslant 1}$ are distinct and that $\liminf _{n \rightarrow \infty}\left|\pi_{i}\left(x_{n}\right)\right|=\liminf _{n \rightarrow \infty}\left|\pi_{j}\left(y_{n}\right)\right|$ $=+\infty$.

Then there exists a sequence $\left(\Theta_{m}\right)_{m \geqslant 1}$ of elements of $\mathrm{Aut}_{1}\left(\mathbf{C}^{p}\right)$ such that $\Theta_{m} \underset{m \rightarrow \infty}{\stackrel{\sigma}{\rightarrow}} I$ and such that $\Theta_{m}\left(x_{n}\right)=y_{n}$ eventually for each fixed $m \geqslant 1$.

Proof. First assume that $i \neq j$. For $n \geqslant 1$, define $z_{n} \in \mathbf{C}^{p}$ by the conditions $\pi_{i}\left(z_{n}\right)=\pi_{i}\left(x_{n}\right), \pi_{(i)}\left(z_{n}\right)=\pi_{(i)}\left(y_{n}\right)$. It follows from Lemma 7.7 that there exist two sequences $\left(\varphi_{m}\right)$ and $\left(\Psi_{m}\right)$ of elements of $\operatorname{Aut}_{1}\left(\mathbf{C}^{p}\right)$ such that $\varphi_{m} \underset{m \rightarrow \infty}{\stackrel{\sigma}{\rightarrow}} I$, $\Psi_{m} \underset{m \rightarrow \infty}{\stackrel{\sigma}{\rightarrow}} I$, and such that $\varphi_{m}\left(x_{n}\right)=z_{n}$ and $\Psi_{m}\left(z_{n}\right)=y_{n}$. So the sequence $\left(\Theta_{m}\right)=\left(\Psi_{m} \circ \varphi_{m}\right)$ satisfies the desired conditions in this case. The general case follows, since when $i=j$ we can use an auxiliary sequence $\left(u_{n}\right)$ satisfying $\pi_{k}\left(u_{n}\right)=n(n \geqslant 1)$ with $k \neq i$.

LEMMA 7.9. Let $\left(\left(x_{q, n}\right)_{n \geqslant 1}\right)_{q \geqslant 1}$ be a countable family of sequences of elements of $\mathbf{C}^{p}$ such that lim $\sup _{n \rightarrow \infty}\left|x_{q, n}\right|=+\infty(q \geqslant 1)$.

Then there exists a sequence $\left(\Theta_{m}\right)$ of elements of $\mathrm{SL}_{p}(\mathbf{C})$ such that $\Theta_{m} \underset{m \rightarrow \infty}{\stackrel{\sigma}{\rightarrow}} I$ and such that $\lim \sup _{n \rightarrow \infty}\left(\inf _{i \leqslant p}\left|\left(\pi_{i} \circ \Theta_{m}\right)\left(x_{q, n}\right)\right|\right)=+\infty$ for every $q \geqslant 1$.

Proof. For $q \geqslant 1$ denote by $\Omega_{q}$ the set

$$
\left\{T \in \mathrm{SL}_{p}(\mathbf{C}) \mid \limsup _{n \rightarrow \infty}\left(\inf _{i \leqslant p}\left|\left(\pi_{i} \circ T\right)\left(x_{q, n}\right)\right|\right)=+\infty\right\} \text {. }
$$

Then $\Omega_{q}=\bigcap_{m \geqslant 1}\left(\cup_{n \geqslant 1}\left\{T \in \mathrm{SL}_{p}(\mathbf{C})\left|\inf _{i \leqslant p}\right|\left(\pi_{i} \circ T\right)\left(x_{q, n}\right) \mid>m\right\}\right)$, so that $\Omega_{q}$ is a $G_{\delta}$ subset of $\mathrm{SL}_{p}(\mathbf{C})$. But if $T \in \mathrm{SL}_{p}(\mathbf{C})$, $\lim _{\sup _{n \rightarrow \infty}}\left|T\left(x_{q, n}\right)\right|=+\infty$, and it follows from Lemma 7.3 that there exists a sequence $\left(\Theta_{m}\right)$ of elements of $\mathrm{SL}_{p}(\mathbf{C})$ such that $\Theta_{m} \underset{m \rightarrow \infty}{\stackrel{\sigma}{\rightarrow}} I$ and such that $\Theta_{m} \circ T \in \Omega_{q}(m \geqslant 1)$. So $\Omega_{q}$ is dense in $\mathrm{SL}_{p}(\mathbf{C})(q \geqslant 1)$ and it follows from the category theorem that the set $\cap_{q \geqslant 1} \Omega_{q}$ is dense in $\mathrm{SL}_{p}(\mathbf{C})$. The result follows. 
THEOREM 7.10. Let $F: \mathbf{C}^{p} \rightarrow \mathbf{C}^{p}$ be a one-to-one entire function and let $S$ be an unbounded subset of $\mathbf{C}^{p}$, with $p \geqslant 2$. Then there exists a sequence $\left(\Theta_{m}\right)$ of

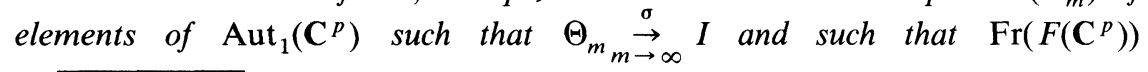
$\subset \overline{\left(F \circ \Theta_{m}\right)(S)}(m \geqslant 1)$.

Proof. Let $\left(\boldsymbol{\alpha}_{q}\right)_{q \geqslant 1}$ be a dense sequence in $\operatorname{Fr}\left(F\left(\mathbf{C}^{p}\right)\right)$. For each $q \geqslant 1$ there exists a sequence $\left(x_{q, n}\right)$ of elements of $\mathbf{C}^{p}$ such that $\alpha_{q}=\lim _{n \rightarrow \infty} F\left(x_{q, n}\right)$, and $\left|x_{q, n}\right|_{n \rightarrow \infty}^{\rightarrow} \infty$ for each $q \geqslant 1$. Fix $m \geqslant 1$. It follows from Lemma 7.9 that there exists $\varphi_{m} \in \mathrm{SL}_{p}(\mathrm{C})$ such that $\left\|\varphi_{m}-I\right\|<1 / m$ and such that $\lim \sup _{n \rightarrow \infty}\left|\left(\pi_{1} \circ \varphi_{m}\right)\left(x_{q, n}\right)\right|=+\infty(q \geqslant 1)$.

Define by induction a family $\left(\omega_{q, m}\right)_{q \geqslant 1}$ of strictly increasing functions from $\mathbf{N}$ into itself satisfying the following conditions.

$$
\left|\left(\pi_{1} \circ \varphi_{m}\right)\left(x_{q, \omega_{q, m}(n)}\right)\right|>\left|\left(\pi_{1} \circ \varphi_{m}\right)\left(x_{q^{\prime}, \omega_{q^{\prime}, m}\left(n^{\prime}\right)}\right)\right|
$$

If $q+n>q^{\prime}+n^{\prime}$ or if $q+n=q^{\prime}+n^{\prime}, q>q^{\prime}$.

$$
\left|\left(\pi_{1} \circ \varphi_{m}\right)\left(x_{q, \omega_{q, m}(n)}\right)\right|>q+n \quad(n \geqslant 1, q \geqslant 1) .
$$

Then all the terms of the double sequence $\left(\left(\pi_{1} \circ \varphi_{m}\right)\left(x_{q, \omega_{q, m}(n)}\right)\right)_{q \geqslant 1, n \geqslant 1}$ are distinct and this double sequence has no limit point in $\mathbf{C}$. There exists for some $i \leqslant p$ a sequence $\left(y_{n}\right)$ of elements of $S$ such that $\left|\pi_{i}\left(y_{n}\right)\right|_{n \rightarrow \infty} \infty$ and such that all the terms of the sequence $\left(\pi_{i}\left(y_{n}\right)\right)$ are distinct. We can rewrite the sequence $\left(y_{n}\right)$ as a double sequence $\left(z_{q, n}\right)_{q \geqslant 1, n \geqslant 1}$. It follows from Corollary 7.8 that there exists $\Psi_{m} \in$ Aut $_{1}\left(\mathbf{C}^{p}\right)$ such that $\left|\Psi_{m}(z)-z\right|<1 / m(|z| \leqslant m)$ and such that $\Psi_{m}\left(z_{q, n}\right)=\varphi_{m}\left(x_{q, \omega_{q, m}(n)}\right)$ for $(q, n) \in \mathbf{N}^{2} \backslash \Lambda_{m}$ where $\Lambda_{m}$ is a finite subset of $\mathbf{N}^{2}$. Set $\Theta_{m}=\varphi_{m}^{-1} \circ \Psi_{m}$. Then $\Theta_{m} \underset{m \rightarrow \infty}{\stackrel{\sigma}{\rightarrow}} I$. Also for every $q \geqslant 1$, we have

$$
\alpha_{q}=\lim _{n \rightarrow \infty} F\left(x_{q, \omega_{q, m}(n)}\right)=\lim _{n \rightarrow \infty}\left(F \circ \Theta_{m}\right)\left(z_{q, n}\right) .
$$

So $\operatorname{Fr}\left(F\left(\mathbf{C}^{p}\right)\right) \subset \overline{\left(F \circ \Theta_{m}\right)(S)}(m \geqslant 1)$, and the theorem is proved.

COROllary 7.11. Let $F: \mathbf{C}^{p} \rightarrow \mathbf{C}^{p}$ be a one-to-one entire function, and let $K$ be a compact subset of $F\left(\mathbf{C}^{p}\right)$, where $p \geqslant 2$. Then there exists a Bieberbach function $G$ such that $(F \circ G)\left(\mathbf{C}^{p}\right) \cap K=\varnothing, \operatorname{Fr}\left(F\left(\mathbf{C}^{p}\right)\right) \subset \operatorname{Fr}\left((F \circ G)\left(\mathbf{C}^{p}\right)\right)$.

Proof. The set $F^{-1}(K)$ is a compact subset of $\mathbf{C}^{p}$. Let $\Omega$ be a compact neighborhood of $F^{-1}(K)$. There exists a Bieberbach function $H$ whose range misses a neighborhood of the origin, and setting $G(z)=n H(z / n)\left(z \in \mathbf{C}^{p}\right)$ with $n$ large enough we obtain a Bieberbach function $G$ such that $G\left(\mathbf{C}^{p}\right) \cap \Omega$ $=\varnothing$. Since $G\left(\mathbf{C}^{p}\right)$ is unbounded it follows from the Theorem that there exists a sequence $\left(\Theta_{m}\right)$ of elements of $\operatorname{Aut}_{1}\left(\mathbf{C}^{p}\right)$ such that $\Theta_{m} \underset{m \rightarrow \infty}{\stackrel{\sigma}{\rightarrow}} I$ and such that $\operatorname{Fr}\left(F\left(\mathbf{C}^{p}\right)\right) \subset \overline{\left(F \circ \Theta_{m} \circ G\right)\left(\mathbf{C}^{p}\right)}(m \geqslant 1)$. Since $\left(F \circ \Theta_{m} \circ G\right)\left(\mathbf{C}^{p}\right) \subset F\left(\mathbf{C}^{p}\right)$, we obtain

$$
\operatorname{Fr}\left(F\left(\mathbf{C}^{p}\right)\right) \subset \operatorname{Fr}\left(\left(F \circ \Theta_{m} \circ G\right)\left(\mathbf{C}^{p}\right)\right) \quad(m \geqslant 1) .
$$


Since $\Theta_{m} \underset{m \rightarrow \infty}{\stackrel{\sigma}{\rightarrow}} I, \Theta_{m}^{-1} \underset{m \rightarrow \infty}{\stackrel{\sigma}{\rightarrow}} I$. So $\Theta_{m}^{-1}\left(F^{-1}(K)\right) \subset \Omega$, hence $\left(\Theta_{m} \circ G\right)\left(\mathbf{C}^{p}\right) \cap$ $F^{-1}(K)=\varnothing$ for large values of $m$. So the Bieberbach function $\Theta_{m} \circ G$ satisfies the desired condition for large values of $m$.

Corollary 7.12. Let $F_{1}: \mathbf{C}^{p} \rightarrow \mathbf{C}^{p}$ be a one-to-one entire function. There exists a sequence $\left(F_{n}\right)_{n \geqslant 2}$ of Bieberbach functions such that

$$
\bigcap_{n \geqslant 1}\left(F_{1} \circ \cdots \circ F_{n}\right)\left(\mathbf{C}^{p}\right)
$$

has empty interior and such that

$$
\operatorname{Fr}\left(\left(F_{1} \circ \cdots \circ F_{n}\right)\left(\mathbf{C}^{p}\right)\right) \subset \operatorname{Fr}\left(\left(F_{1} \circ \cdots \circ F_{n+1}\right)\left(\mathbf{C}^{p}\right)\right) \quad(n \geqslant 1) .
$$

Proof. By a repeated use of Corollary 7.11 we can construct by induction a sequence $\left(F_{n}\right)_{n \geqslant 2}$ of Bieberbach functions such that

$$
\operatorname{Fr}\left[\left(F_{1} \circ \cdots \circ F_{n}\right)\left(\mathbf{C}^{p}\right)\right] \subset \operatorname{Fr}\left[\left(F_{1} \circ \cdots \circ F_{n+1}\right)\left(\mathbf{C}^{p}\right)\right] \quad(n \geqslant 1)
$$

and such that $\left(F_{1} \circ \cdots \circ F_{n}\right)\left(\mathbf{C}^{p}\right) \cap K_{n-1}=\varnothing(n \geqslant 2)$, where

$K_{i}=\left\{z \in\left(F_{1} \circ \cdots \circ F_{i}\right)\left(\mathbf{C}^{p}\right)\left|d\left(z, \operatorname{Fr}\left[\left(F_{1} \circ \cdots \circ F_{i}\right)\left(\mathbf{C}^{p}\right)\right]\right) \geqslant \frac{1}{i},\right| z \mid \leqslant i\right\}$.

Clearly, the interior of $\bigcap_{n \geqslant 1}\left(F_{1} \circ \cdots \circ F_{n}\right)\left(\mathbf{C}^{p}\right)$ is empty, and the sequence $\left(F_{n}\right)_{n \geqslant 2}$ has the required properties.

A natural idea for constructing a sequence $\left(F_{n}\right)_{n \geqslant 1}$ of entire mappings $\mathbf{C}^{p} \rightarrow \mathbf{C}^{p}$ such that $\bigcap_{n \geqslant 1}\left(F_{1} \circ \cdots \circ F_{n}\right)\left(\mathbf{C}^{p}\right)=\varnothing$ would be to try to construct by induction the sequence $\left(F_{n}\right)$ so that $\left(F_{1} \circ \cdots \circ F_{n}\right)\left(\mathbf{C}^{2}\right) \cap D(0, n)=\varnothing$ $(n \geqslant 1)$. When $p=2$ we can take $F_{1}=\Theta \circ F \circ \Psi$, where $F$ is any of the functions constructed in $\S 6$ and where $\Theta$ and $\Psi$ are suitable affine automorphisms of $\mathbf{C}^{2}$ (for all examples except Fatou's a suitable choice of $\Theta$ and $\Psi$ even ensures that $F_{1}$ is a Bieberbach function). But the following result shows that Bieberbach's original function and Sadullaev's functions would be a bad start for such a construction (the notations below are the same as in $\S 6$, so that $k \in \mathbf{C}, \quad|k|>1, \quad j \in \mathbf{C}[X], d^{0} j \geqslant 2, \quad j(0)=j^{\prime}(0)=0$, and $K>0$ satisfies $|j(\xi)| \geqslant(k+1)|\xi|$ whenever $\xi \in \mathbf{C},|\xi| \geqslant K)$.

THEOREM 7.13. (i) For every $q \geqslant 1$ and for every nonconstant entire mapping $H: \mathbf{C}^{q} \rightarrow \mathbf{C}^{2}$ we have $\left(G_{k, j} \circ H\right)\left(\mathbf{C}^{q}\right) \cap D(0, K) \neq \varnothing$.

(ii) For every sequence $\left(F_{n}\right)$ of nondegenerate entire mappings from $\mathbf{C}^{2}$ into itself, we have

$$
\pi_{i}\left(\bigcap_{n \geqslant 1} \overline{\left(G_{k, j} \circ F_{1} \circ \cdots \circ F_{n}\right)\left(\mathbf{C}^{2}\right)}\right)=\mathbf{C} \quad(i=1,2) .
$$

(iii) For every sequence $\left(F_{n}\right)$ of entire mappings from $\mathbf{C}^{2}$ into itself we have $\bigcap_{n \geqslant 1} \overline{\left(G_{k, j} \circ F_{1} \circ \cdots \circ F_{n}\right)\left(\mathbf{C}^{2}\right)} \neq \varnothing$.

Proof. It follows from Proposition 6.7(i) that $\left|z_{1}\right|<\max \left(K,\left|z_{2}\right|\right)$ for every $z=\left(z_{1}, z_{2}\right) \in G_{k, j}\left(\mathbf{C}^{2}\right)$. If $\left(G_{k, j} \circ H\right)\left(\mathbf{C}^{q}\right) \cap D(0, K)=\varnothing$ we have

$$
\left|\left(\pi_{1} \circ G_{k, j} \circ H\right)(z)\right|<\max \left(K,\left|\left(\pi_{2} \circ G_{k, j} \circ H\right)(z)\right|\right)
$$


and

$$
\max \left(\left|\left(\pi_{1} \circ G_{k, j} \circ H\right)(z)\right|,\left|\left(\pi_{2} \circ G_{k, j} \circ H\right)(z)\right|\right) \geqslant K
$$

so that $\left|\left(\pi_{2} \circ G_{k, j} \circ H\right)(z)\right| \geqslant K\left(z \in \mathbf{C}^{q}\right)$. It follows then from Picard's theorem that $\pi_{2} \circ G_{k, j} \circ H$ is constant. So $\pi_{1} \circ G_{k, j} \circ H$ is bounded, hence constant too. Since $G_{k, j}$ is one to one, $H$ is constant, which proves (i).

To prove (ii), notice that $F_{1} \circ \cdots \circ F_{n}$ is nonconstant for every $n \geqslant 1$. Hence $\pi_{2} \circ G_{k, j} \circ F_{1} \circ \cdots \circ F_{n}$ is not constant, by the same argument as above. Since the sequence $\left(G_{k, j} \circ F_{1} \circ \cdots \circ F_{n}\right)\left(\mathbf{C}^{2}\right)$ is decreasing there exists $\alpha \in \mathbf{C}$ such that $\mathbf{C} \backslash\{\alpha\} \subset\left(\pi_{2} \circ G_{k, j} \circ F_{1} \circ \cdots \circ F_{n}\right)\left(\mathbf{C}^{2}\right)(n \geqslant 1)$, by Picard's theorem. So for every $\xi \in \mathbf{C} \backslash\{\alpha\}$ and every $n \geqslant 1$ there exists $x_{n}(\xi) \in \mathbf{C}$ such that $\left(x_{n}(\xi), \xi\right) \in\left(G_{k, j} \circ F_{1} \circ \cdots \circ F_{n}\right)\left(\mathbf{C}^{2}\right)$. Also $\left|x_{n}(\xi)\right|<\max (K,|\xi|)$ so the sequence $\left(x_{n}(\xi)\right)$ possesses at least one limit point $x(\xi)$ for every $\xi \in \mathbf{C} \backslash\{\alpha\}$. Since the sequence $\left(G_{k, j} \circ F_{1} \circ \ldots \circ F_{n}\right)\left(\mathbf{C}^{2}\right)$ is decreasing we have

$$
(x(\xi), \xi) \in \overline{\left(G_{k, j} \circ F_{1} \circ \cdots \circ F_{n}\right)\left(\mathbf{C}^{2}\right)} \quad(n \geqslant 1, \xi \neq \alpha) .
$$

But since $|x(\xi)| \leqslant \max (K,|\xi|)(\xi \in \mathbf{C} \backslash\{\alpha\})$, there exists $x(\alpha) \in \mathbf{C}$ such that

$$
(x(\alpha), \alpha) \in \bigcap_{n \geqslant 1} \overline{\left(G_{k, j} \circ F_{1} \circ \cdots \circ F_{n}\right)\left(\mathbf{C}^{2}\right)},
$$

and

$$
\pi_{2}\left(\bigcap_{n \geqslant 1} \overline{\left(G_{k, j} \circ F_{1} \circ \cdots \circ F_{n}\right)\left(\mathbf{C}^{2}\right)}\right)=\mathbf{C} .
$$

The fact that $\pi_{1}\left(\bigcap_{n \geqslant 1} \overline{\left(G_{k, j} \circ F_{1} \circ \cdots \circ F_{n}\right)\left(\mathbf{C}^{2}\right)}\right)=\mathbf{C}$ follows from Proposition 6.7 (ii) by similar arguments. To prove (ii) we only used the fact that $F_{1} \circ \cdots \circ F_{n}$ is nonconstant for every $n \geqslant 1$. But if $F_{1} \circ \cdots \circ F_{n}$ is constant for some $n \geqslant 1, \overline{\left(G_{k, j} \circ F_{1} \circ \cdots \circ F_{m}\right)\left(\mathbf{C}^{2}\right)}$ reduces to the same singleton for every $m \geqslant n$, and the fact that assertion (iii) holds is obvious in that case.

We conclude this section with the following result, which follows also from Proposition 6.7.

THEOREM 7.14. For every $\varepsilon>0$, there exists a Bieberbach function $F$ over $\mathbf{C}^{2}$ which possesses the following properties.

(i) $F\left(\mathbf{C}^{2}\right) \cap \overline{D(0,1)}=\varnothing$.

(ii) For every $q \geqslant 1$ and for every nonconstant entire mapping $H: \mathbf{C}^{q} \rightarrow \mathbf{C}^{2}$, $(F \circ H)\left(\mathbf{C}^{q}\right) \cap D(0,1+\varepsilon) \neq \varnothing$.

Proof. Set $G=G_{2, X^{2}}$, say, so that we can take $K=3$. We may assume without loss of generality that $\varepsilon<1$, and $G$ is a Bieberbach function. Also $\left|z_{1}\right|<\max \left(3,\left|z_{2}\right|\right)$ for every $z=\left(z_{1}, z_{2}\right) \in G\left(\mathbf{C}^{2}\right)$. Set $a=\varepsilon / 9, b=1 / 3$, and $G_{1}=\Theta \circ G \circ \Theta^{-1}$, where $\Theta$ is the linear map $\left(z_{1}, z_{2}\right) \rightarrow\left(a z_{1}, b z_{2}\right)$. Then $G_{1}\left(\mathbf{C}^{2}\right)=(\Theta \circ G)\left(\mathbf{C}^{2}\right)$ so that

$$
\left|z_{1}\right|<\max \left(\varepsilon / 3, \varepsilon\left|z_{2}\right| / 3\right)
$$


for every $\left(z_{1}, z_{2}\right) \in G_{1}\left(\mathbf{C}^{2}\right)$. Consider the polydisc

$$
\Delta=\left\{\left(z_{1}, z_{2}\right) \in \mathbf{C}^{2}|| z_{1}-1-2 \varepsilon / 3|\leqslant 1,| z_{2}-1 \mid \leqslant 1\right\} .
$$

If $\left(z_{1}, z_{2}\right) \in \Delta$ then $\left|z_{1}\right| \geqslant 2 \varepsilon / 3$ and $\left|z_{2}\right| \leqslant 2$ so that $\Delta \cap G_{1}\left(\mathbf{C}^{2}\right)=\varnothing$. Now consider an entire mapping $H: \mathbf{C}^{q} \rightarrow \mathbf{C}^{2}$ such that $\sup \left(\left|z_{1}-1-2 \varepsilon / 3\right|\right.$, $\left.\left|z_{2}-1\right|\right) \geqslant 1+\varepsilon$ for every $\left(z_{1}, z_{2}\right) \in\left(G_{1} \circ H\right)\left(\mathbf{C}^{q}\right)$. Then $\left|z_{1}\right| \geqslant \varepsilon / 3$ or $\left|z_{2}\right| \geqslant \varepsilon$ for every $\left(z_{1}, z_{2}\right) \in\left(G_{1} \circ H\right)\left(\mathbf{C}^{q}\right)$. In the first case we must have $\left|z_{2}\right| \geqslant 1$, so that $\left(\pi_{2} \circ G_{1} \circ H\right)\left(\mathbf{C}^{q}\right)$ is not dense in C. So $\pi_{2} \circ G_{1} \circ H$ is constant and $\pi_{1} \circ G_{1} \circ H$ is bounded, hence constant too. So $H$ is constant if $\left(G_{1} \circ H\right)\left(\mathbf{C}^{q}\right)$ avoids the polydisc of radius $1+\varepsilon$ centered at the point $(1+2 \varepsilon / 3,1)$. Denote by $\tau$ the translation $\left(z_{1}, z_{2}\right) \rightarrow\left(z_{1}-1-2 \varepsilon / 3, z_{2}-1\right)$. Then $F=\tau \circ G_{1}$ possesses the required properties.

We did not investigate whether there is a bounded set $B$ such that $G^{-1}(B)$ meets the range of any nondegenerate (or nonconstant) entire mapping $H: \mathrm{C}^{2} \rightarrow \mathrm{C}^{2}$ in the case where $G$ is Fatou's original function, or Kodaira's function.

In fact we do not know for any $p \geqslant 2$ whether there exists a nondegenerate function $G: \mathbf{C}^{p} \rightarrow \mathbf{C}^{p}$ with nondense range such that for every bounded subset $B$ of $\mathbf{C}^{p}$ there exists a nondegenerate entire function $H_{B}: \mathbf{C}^{p} \rightarrow \mathbf{C}^{p}$ satisfying $H_{B}\left(\mathbf{C}^{p}\right) \cap G^{-1}(B)=\varnothing$ (for a description of classes of sets which meet the range of any nondegenerate entire mapping $\mathbf{C}^{p} \rightarrow \mathbf{C}^{p}$ we refer again to Carlson and Griffiths [21], Griffiths [51] and Gruman [52]).

Note that if $F: \mathbf{C}^{p} \rightarrow \mathbf{C}^{p}$ is nondegenerate, and if $F\left(\mathbf{C}^{p}\right)$ is contained in a pseudoconvex open set $U$ such that

$$
\liminf _{\substack{|z| \rightarrow \infty \\ z \in F\left(\mathbf{C}^{p}\right)}} d(z, \operatorname{Fr} U)>0,
$$

then there exists $K>0$ such that $(F \circ G)\left(\mathbf{C}^{p}\right) \cap D(0, K) \neq \varnothing$ for every nondegenerate mapping $G: \mathbf{C}^{p} \rightarrow \mathbf{C}^{p}$. To see this, note that the function $z \rightarrow-\log (d(F[G(z)], \operatorname{Fr} U))$ is plurisubharmonic and nonconstant over $\mathbf{C}^{p}$, hence unbounded. So there exists a sequence $\left(z_{n}\right)$ such that $d\left(F\left[G\left(z_{n}\right)\right]\right.$, $\operatorname{Fr} U) \rightarrow 0$ and $\left|F\left[G\left(z_{n}\right)\right]\right| \leqslant K$ eventually if

$$
\inf _{\substack{|z|>K \\ z \in F\left(\mathbf{C}^{p}\right)}} d(z, \operatorname{Fr} U)>0 .
$$

But we were not able to find a concrete example of such a pair $(F, U)$.

8. New examples of Bieberbach functions. In this section we present a new method of constructing Bieberbach functions. The method is suggested by the results of $\S 5$, and consists in producing sequences $\left(\Theta_{n}\right)_{n \geqslant 1}$ of elements of Aut $_{1}\left(\mathbf{C}^{2}\right)$ which converge uniformly on compact sets to a Bieberbach function $F$ and satisfy $\left|\Theta_{n}^{-1}(z)\right|_{n \rightarrow \infty} \infty(z \in \Delta)$, where $\Delta$ is some "large" set, so that $F\left(\mathbf{C}^{2}\right) \cap \Delta=\varnothing$. We thus obtain a Bieberbach function $F_{1}$ such that $F_{1}\left(\mathbf{C}^{2}\right)$ $\cap\left(U_{1} \cup V_{1}\right)=\varnothing$ and a Bieberbach function $F_{2}$ such that $V_{1} \subset F_{2}\left(\mathbf{C}^{2}\right)$, $F_{2}\left(\mathbf{C}^{2}\right) \cap U_{1}=\varnothing$, where $U_{1}=\left\{(x, y) \in \mathbf{C}^{2} \mid \inf (\operatorname{Re} x, \operatorname{Re} y) \geqslant 1\right\}, V_{1}=-U_{1}$. This leads to the construction of a nondegenerate entire function $G_{\varepsilon}: \mathbf{C}^{2} \rightarrow \mathrm{C}^{2}$ 
such that

$$
\begin{gathered}
G_{\varepsilon}\left(\mathbf{C}^{2}\right) \cap D(0,1-\varepsilon)=\varnothing, \\
\inf \left(\left|\pi_{1}(z)\right|,\left|\pi_{2}(z)\right|\right) \leqslant 1 \quad\left(z \in G_{\varepsilon}\left(\mathbf{C}^{2}\right)\right), \\
\lim _{\substack{|z| \rightarrow \infty \\
z \in G_{\varepsilon}\left(\mathbf{C}^{2}\right)}}\left[\inf \left(\left|\pi_{1}(z)\right|,\left|\pi_{2}(z)\right|\right)\right]=0,
\end{gathered}
$$

where $\varepsilon$ is any given element of $(0,1)$.

Heuristically, this means that there exist nondegenerate entire functions over $\mathrm{C}^{2}$ whose ranges are very "small". The second condition implies of course that $\left(G_{\varepsilon} \circ H\right)\left(\mathbf{C}^{2}\right) \cap D(0,2) \neq \varnothing$ for every nonconstant entire function $H: \mathbf{C}^{2} \rightarrow$ $\mathrm{C}^{2}$, but similar results were obtained in $\S 7$ for some Bieberbach functions.

In the other direction we construct for every $\varepsilon>0$ an entire function $H_{\varepsilon}: \mathbf{C}^{2} \rightarrow \mathbf{C}^{2}$ such that $H_{\varepsilon}\left(\mathbf{C}^{2}\right) \cap \overline{D(0,1)}=\varnothing$ and such that $\mathbf{C}^{2} \backslash H_{\varepsilon}\left(\mathbf{C}^{2}\right) \subset$ $D(0,1+\varepsilon)$. Moreover, the number of elements of $H_{\varepsilon}^{-1}(\{z\})$ is bounded for every $z \in \mathbf{C}^{2}$ by an integer which depends on $\varepsilon$ (of course, as observed in $\S 4$, these functions $H_{\varepsilon}$ cannot be one-to-one).

The key to the constructions is a deep theorem of Arakelian about entire functions of 'one complex variable. Denote by $\mathbf{C}_{\infty}=\mathbf{C} \cup\{\infty\}$ the Riemann sphere, and let $V$ be a closed subset of $\mathbf{C}$ such that $\mathbf{C}_{\infty} \backslash V$ is connected and locally connected at $\{\infty\}$. Arakelian's theorem (see [6 or 48, Theorem 1]) shows that any function $f: V \rightarrow \mathbf{C}$ which is continuous over $V$ and analytic over $\stackrel{\circ}{V}$ can be uniformly approximated over $V$ by entire functions. In fact if $\omega$ is a positive-valued continuous function over $\mathbf{R}^{+}$such that

$$
\int_{1}^{\infty} \frac{|\log \omega(t)|}{t^{3 / 2}} d t<+\infty
$$

it follows from a result of Arakelian and Gauthier [7, Corollary 5] that there exists an entire function $g$ over $\mathbf{C}$ such that $|f(z)-g(z)| \leqslant \omega(|z|)$ for every $z \in V$. This leads to the following lemma.

LEMMA 8.1. Let $\left(a_{1}, \ldots, a_{2 n}\right)$ be a strictly increasing family of real numbers, and let $\alpha_{1}, \ldots, \alpha_{n+1}$ be complex numbers. Set $V_{1}=\left\{z \in \mathbf{C} \mid \operatorname{Re} z \leqslant a_{1}\right\}, V_{i}=$ $\left\{z \in \mathbf{C} \mid a_{2 i-2} \leqslant \operatorname{Re} z \leqslant a_{2 i-1}\right\} \quad(2 \leqslant i \leqslant n), \quad V_{n+1}=\left\{z \in \mathbf{C} \mid \operatorname{Re} z \geqslant a_{2 n}\right\}$. Then there exists a sequence $\left(f_{m}\right)$ of entire functions over $\mathbf{C}$ such that

$$
\sup _{z \in V_{i}}\left|f_{m}(z)-\alpha_{i}\right| e^{\sqrt[4]{|z|}} \operatorname{mon}_{m \rightarrow \infty} 0 \quad(1 \leqslant i \leqslant n+1) .
$$

Proof. Set $V=V_{1} \cup \cdots \cup V_{n+1}$. Then $\mathbf{C}_{\infty} \backslash V$ is connected and is locally connected at $\{\infty\}$. Now define $g: V \rightarrow \mathbf{C}$ by the conditions $g\left(V_{i}\right)=\left\{\alpha_{i}\right\}$ $(1 \leqslant i \leqslant n+1)$. It follows from the Arakelian-Gauthier theorem that there exists for each $m \geqslant 1$ an entire function $f_{m}$ over $\mathbf{C}$ satisfying $\left|f_{m}(z)-g(z)\right| \leqslant$ $e^{-|z|^{1 / 4}} / m$ for every $z \in V$, and the result follows.

For $a \in \mathbf{R}$ set $U_{a}=\left\{z \in \mathbf{C}^{2} \mid \inf \left[\operatorname{Re} \pi_{1}(z), \operatorname{Re} \pi_{2}(z)\right] \geqslant a\right\}$. The following lemma is a crude application of Lemma 8.1. 
LEMMA 8.2. Let $a \geqslant 0$. There exists a sequence $\left(\Theta_{m}\right)$ of elements of $\mathrm{Aut}_{1}\left(\mathrm{C}^{2}\right)$ which possesses the following properties.

(1) $\sup _{|z| \leqslant a}\left[\left|\Theta_{m}(z)-z\right|+\left|\Theta_{m}^{-1}(z)-z\right|\right]_{m \rightarrow \infty}^{\rightarrow} 0$.

(2) $\Theta_{m}\left(U_{a+1}\right) \subset U_{a+2}, \Theta_{m}\left(-U_{a+1}\right) \subset-U_{a+2}(m \geqslant 1)$.

Proof. We apply Lemma 8.1 with

$$
\begin{gathered}
a_{1}=-a-1, \quad a_{2}=-a-\frac{1}{2}, \quad a_{3}=a+\frac{1}{2}, \quad a_{4}=a+1, \\
\alpha_{1}=-2, \quad \alpha_{2}=0, \quad \alpha_{3}=2 .
\end{gathered}
$$

Let $\left(f_{m}\right)$ be the sequence of entire functions given by the lemma. We may assume that $\operatorname{Re} f_{m}(\xi)>1(\operatorname{Re} \xi \geqslant a+1), \operatorname{Re} f_{m}(\xi)<-1(\operatorname{Re} \xi \leqslant-a-1)$, $\left|f_{m}(\xi)\right|<\frac{1}{2}\left(|\operatorname{Re} \xi| \leqslant a+\frac{1}{2}\right)$ for every $m \geqslant 1$.

Define $\Theta_{m}: \mathbf{C}^{2} \rightarrow \mathbf{C}^{2}$ by the formula $\Theta_{m}\left(z_{1}, z_{2}\right)=\left(u_{1}, u_{2}\right)$ where $u_{1}=z_{1}+$ $f_{m}\left(z_{2}\right), u_{2}=z_{2}+f_{m}\left(u_{1}\right)$. Then $\Theta_{m} \in \operatorname{Aut}_{1}\left(\mathbf{C}^{2}\right)$. Also $\Theta_{m}^{-1}\left(u_{1}, u_{2}\right)=\left(z_{1}, z_{2}\right)$ where $z_{2}=u_{2}-f_{m}\left(u_{1}\right), z_{1}=u_{1}-f_{m}\left(z_{2}\right)$. If $\left(z_{1}, z_{2}\right) \in U_{a+1}$ then $\operatorname{Re} u_{1} \geqslant$ $a+2$, hence $\operatorname{Re} f_{m}\left(u_{1}\right) \geqslant 1$, and so $\operatorname{Re} u_{2} \geqslant a+2$ and $\Theta_{m}\left(U_{a+1}\right) \subset U_{a+2}$. Similarly $\Theta_{m}\left(-U_{a+1}\right) \subset-U_{a+2}$. Now if $\left|\left(z_{1}, z_{2}\right)\right| \leqslant a$ then $\left|\operatorname{Re} u_{1}\right| \leqslant a+\frac{1}{2}$ and so

$$
\left|\Theta_{m}\left(z_{1}, z_{2}\right)-\left(z_{1}, z_{2}\right)\right| \leqslant \sup _{|\operatorname{Re} \xi| \leqslant a+1 / 2}\left|f_{m}(\xi)\right| .
$$

Also $\left|\operatorname{Re} f_{m}\left(z_{1}\right)\right| \leqslant \frac{1}{2},\left|\operatorname{Re}\left(z_{2}-f_{m}\left(z_{1}\right)\right)\right| \leqslant a+\frac{1}{2}$ and so

$$
\left|\Theta_{m}^{-1}\left(z_{1}, z_{2}\right)-\left(z_{1}, z_{2}\right)\right| \leqslant \sup _{|\operatorname{Re} \xi| \leqslant a+1 / 2}\left|f_{m}(\xi)\right| .
$$

So the sequence $\left(\Theta_{m}\right)_{m \geqslant 1}$ satisfies the desired conditions.

For $m \geqslant 1, F \in \mathscr{H}\left(\mathbf{C}^{p}, \mathbf{C}^{p}\right)$, we set as before $\|F\|_{m}=\sup _{|z| \leqslant m}|F(z)|$.

LEMMA 8.3. Let $p \geqslant 1$ and let $\left(F_{n}\right)_{n \geqslant 1}$ be a sequence of entire mappings from $\mathrm{C}^{p}$ into itself. If $\sum_{n=1}^{\infty}\left\|I-F_{n}\right\|_{m}<+\infty$ for every $m \geqslant 1$, then the sequence $\left(F_{1} \circ \cdots \circ F_{n}\right)_{n \geqslant 1}$ converges uniformly on compact subsets of $\mathbf{C}^{p}$ to an entire mapping $F: \mathbf{C}^{p} \rightarrow \mathbf{C}^{p}$.

Proof. Fix $m \geqslant 1$. There exists $n_{1} \geqslant 1$ such that $\sum_{n=n_{1}+1}^{\infty}\left\|I-F_{n}\right\|_{m+1}<1$. Let $z \in \mathbf{C}^{p}$ such that $|z| \leqslant m$. If $n \geqslant n_{1}+1$ then

$$
\left|F_{n}(z)\right| \leqslant|z|+\left\|I-F_{n}\right\|_{m} \leqslant m+\left\|I-F_{n}\right\|_{m+1}<m+1 .
$$

A routine finite induction shows that

$$
\left|\left(F_{k} \circ \cdots \circ F_{n}\right)(z)\right| \leqslant m+\sum_{p=k}^{n}\left\|I-F_{p}\right\|_{m+1}<m+1
$$

$\left(n_{1}+1 \leqslant \mathrm{k} \leqslant n\right)$. Hence $\left\|F_{n_{1}+1} \circ \cdots \circ F_{n}\right\|_{m}<m+1\left(n \geqslant n_{1}+1\right)$ and so $\sup _{n \geqslant 1}\left\|F_{1} \circ \cdots \circ F_{n}\right\|_{m}<+\infty$ for every $m \geqslant 1$. We thus see that the family $\left(F_{1} \circ \cdots \circ F_{n}\right)_{n \geqslant 1}$ is a normal family, and so for every $m \geqslant 1$ there exists $\lambda_{m}>0$ such that

$$
\left\|\left(F_{1} \circ \cdots \circ F_{n}\right)^{\prime}(\xi)\right\| \leqslant \lambda_{m} \quad(|\xi| \leqslant m, n \geqslant 1) .
$$


Consider again $m \geqslant 1$. There exists $p \geqslant m$ such that $\left\|F_{n}\right\|_{m} \leqslant p(n \geqslant 1)$, and so $\left|\left(F_{1} \circ \cdots \circ F_{n}\right)(z)-\left(F_{1} \circ \cdots \circ F_{n+1}\right)(z)\right| \leqslant \lambda_{p}\left|z-F_{n+1}(z)\right| \quad(|z| \leqslant m$, $n \geqslant 1)$. So

$$
\sum_{n=1}^{\infty}\left\|\left(F_{1} \circ \cdots \circ F_{n}\right)-\left(F_{1} \circ \cdots \circ F_{n+1}\right)\right\|_{m}<+\infty \quad(m \geqslant 1)
$$

and the series $F_{1}+\sum_{n=1}^{\infty}\left(F_{1} \circ \cdots \circ F_{n+1}-F_{1} \circ \cdots \circ F_{n}\right)$ converges uniformly on compact subsets of $\mathbf{C}^{p}$ to an entire mapping $F$. We have $F_{1} \circ \cdots \circ F_{n} \underset{n \rightarrow \infty}{\stackrel{\sigma}{\rightarrow}} F$, which proves the lemma.

THEOREM 8.4. There exists a Bieberbach function $F$ over $\mathbf{C}^{2}$ such that $\inf \left(\operatorname{Re} z_{1}, \operatorname{Re} z_{2}\right)<1, \sup \left(\operatorname{Re} z_{1}, \operatorname{Re} z_{2}\right)>-1$ for every $\left(z_{1}, z_{2}\right) \in F\left(\mathbf{C}^{2}\right)$.

Proof. By using Lemma 8.2, we can construct for every $n \geqslant 1$ an element $\varphi_{n}$ of $\operatorname{Aut}_{1}\left(\mathbf{C}^{2}\right)$ such that

$$
\left\|I-\varphi_{n}^{-1}\right\|_{n-1}<2^{-n}, \quad \varphi_{n}\left(U_{n} \cup\left(-U_{n}\right)\right) \subset U_{n+1} \cup\left(-U_{n+1}\right) .
$$

Set $\Phi_{n}=\varphi_{n}^{-1}$. It follows from lemma 8.3 that the sequence $\left(\Phi_{1} \circ \cdots \circ \Phi_{n}\right)_{n \geqslant 1}$ converges uniformly on compact subsets of $\mathbf{C}^{2}$ to an entire mapping $F$.

We have $\left(\Phi_{n}^{-1} \circ \cdots \circ \Phi_{1}^{-1}\right)\left(U_{1} \cup\left(-U_{1}\right)\right) \subset U_{n+1} \cup\left(-U_{n+1}\right)$, and so

$$
\left|\left(\Phi_{n}^{-1} \circ \cdots \circ \Phi^{-1}\right)(z)\right| \underset{n \rightarrow \infty}{\rightarrow} \infty
$$

for every $z \in U_{1} \cup\left(-U_{1}\right)$. So $F\left(\mathbf{C}^{2}\right) \cap\left(U_{1} \cup\left(-U_{1}\right)\right)=\varnothing$. Clearly, $F$ is a Bieberbach function, and the theorem is proved.

COROllary 8.5. Let $\varepsilon \in(0,1)$. There exists an entire function $G_{\varepsilon}: \mathbf{C}^{2} \rightarrow \mathbf{C}^{2}$, with $J\left(G_{\varepsilon}\right)(z) \neq 0$ for every $z \in \mathbf{C}^{2}$, which possesses the following properties.

(1) $G_{\varepsilon}\left(\mathbf{C}^{2}\right) \cap D(0,1-\varepsilon)=\varnothing$.

(2) $\pi_{1}\left(G_{\varepsilon}(z)\right) \neq 0$ and $\pi_{2}\left(G_{\varepsilon}(z)\right) \neq 0$ for every $z \in \mathbf{C}^{2}$.

(3) $\inf \left(\left|u_{1}\right|,\left|u_{2}\right|^{1+\varepsilon}\left|u_{1}\right|\right) \leqslant 1$ and $\inf \left(\left|u_{2}\right|,\left|u_{1}\right|^{1+\varepsilon}\left|u_{2}\right|\right) \leqslant 1$ for every $\left(u_{1}, u_{2}\right) \in$ $G_{\varepsilon}\left(\mathbf{C}^{2}\right)$.

(4) $\inf \left(\left|u_{1}\right|,\left|u_{2}\right|\right) \leqslant 1$ for every $\left(u_{1}, u_{2}\right) \in G_{\varepsilon}\left(\mathbf{C}^{2}\right)$ and

$$
\inf \left(\left|\pi_{1}(u)\right|,\left|\pi_{2}(u)\right|\right) \underset{|u| \rightarrow \infty}{\rightarrow} 0
$$

for every $u \in G_{\varepsilon}\left(\mathbf{C}^{2}\right)$.

Proof. Let $F$ be a Bieberbach function which satisfies the conditions of Theorem 8.4. There exists an affine map $L_{\varepsilon}$ over $\mathbf{C}^{2}$ such that $F_{\varepsilon}=\left(L_{\varepsilon} \circ F\right)\left(\mathbf{C}^{2}\right)$ satisfies the following conditions.

(a) If $\left(z_{1}, z_{2}\right) \in F_{\varepsilon}\left(\mathbf{C}^{2}\right), \operatorname{Re} z_{2} \geqslant 0$ then

$$
(1+\varepsilon) \operatorname{Re} z_{1}+\operatorname{Re} z_{2} \leqslant 0 .
$$

(b) If $\left(z_{1}, z_{2}\right) \in F_{\varepsilon}\left(\mathbf{C}^{2}\right), \operatorname{Re} z_{1} \geqslant 0$ then

$$
(1+\varepsilon) \operatorname{Re} z_{2}+\operatorname{Re} z_{1} \leqslant 0 .
$$

(c) If $\left(z_{1}, z_{2}\right) \in F_{\varepsilon}\left(\mathbf{C}^{2}\right), \operatorname{Re} z_{1} \leqslant 0, \operatorname{Re} z_{2} \leqslant 0$ then

$$
\sup \left(\operatorname{Re} z_{1}, \operatorname{Re} z_{2}\right) \geqslant \log (1-\varepsilon) \text {. }
$$


Set $\varphi(z)=\left(e^{\pi_{1}(z)}, e^{\pi_{2}(z)}\right)\left(z \in \mathbf{C}^{2}\right)$ and set $G_{\varepsilon}=\varphi \circ F_{\varepsilon}$. If $\left(z_{1}, z_{2}\right) \in G_{\varepsilon}\left(\mathbf{C}^{2}\right)$ then $\left(\left|z_{1}\right|,\left|z_{2}\right|\right)=\left(e^{\operatorname{Re} u_{1}}, e^{\operatorname{Re} u_{2}}\right)$, where $\left(u_{1}, u_{2}\right) \in F_{\varepsilon}\left(\mathbf{C}^{2}\right)$. Clearly, $G_{\varepsilon}$ satisfies (1), (2), (3). Assertion (4) is an immediate consequence of (3).

REMARK 8.6. (1) We have $F_{\varepsilon}\left(\mathbf{C}^{2}\right) \cap D(0,1)=\varnothing$ and $\left(F_{\varepsilon} \circ H\right)\left(\mathbf{C}^{q}\right) \cap$ $D(0,1 /(1-2 \varepsilon)) \neq \varnothing$ for every nonconstant entire function $H: \mathbf{C}^{q} \rightarrow \mathbf{C}^{2}$ and every $q \geqslant 1$, where $F_{\varepsilon}=1 /(1-\varepsilon) G_{\varepsilon}$. This follows from the fact that $F_{\varepsilon}\left(\mathbf{C}^{2}\right) \subset\left\{\left(z_{1}, z_{2}\right) \in \mathbf{C}^{2} \mid \inf \left(\left|z_{1}\right|,\left|z_{2}\right|\right) \leqslant 1 /(1-\varepsilon)\right\}$. So if $\left(F_{\varepsilon} \circ H\right)\left(\mathbf{C}^{q}\right) \cap$ $D(0,1 /(1-2 \varepsilon))=\varnothing$ the connected set $\left(F_{\varepsilon} \circ H\right)\left(\mathbf{C}^{q}\right)$ must be contained either in the set $\left\{\left(z_{1}, z_{2}\right) \in \mathbf{C}^{2}|| z_{1}|\leqslant 1 /(1-\varepsilon),| z_{2} \mid \geqslant 1 /(1-2 \varepsilon)\right\}$ or in the set $\left\{\left(z_{1}, z_{2}\right) \in \mathbf{C}^{2}|| z_{2}|\leqslant 1 /(1-\varepsilon),| z_{1} \mid \geqslant 1 /(1-2 \varepsilon)\right\}$. In both cases it follows from Picard's theorem (or simply from Weierstrass's theorem) that $\pi_{1} \circ F_{\varepsilon} \circ H$ and $\pi_{2} \circ F_{\varepsilon} \circ H$ are constant, so that $H$ is constant since $F_{\varepsilon}$ is locally one-to-one. But similar properties were already obtained in Theorem 7.14 for some Bieberbach functions, which are one-to-one.

(2) We can choose the affine map $L_{\varepsilon}$ introduced above so that $F_{\varepsilon}=L_{\varepsilon} \circ F$ satisfies the condition

(c') $(1+\varepsilon) \operatorname{Re} z_{2}+\operatorname{Re} z_{1} \geqslant(2+\varepsilon) \log (1-\varepsilon)$ if $\left(z_{1}, z_{2}\right) \in F_{\varepsilon}\left(\mathbf{C}^{2}\right), \operatorname{Re} z_{1} \leqslant$ $\log (1-\varepsilon)$ and $(1+\varepsilon) \operatorname{Re} z_{1}+\operatorname{Re} z_{2} \geqslant(2+\varepsilon) \log (1-\varepsilon)$ if $\left(z_{1}, z_{2}\right) \in F_{\varepsilon}\left(\mathbf{C}^{2}\right)$, $\operatorname{Re} z_{1} \geqslant \log (1-\varepsilon)$.

So it is possible to obtain in Corollary 8.5 the extra condition

$$
\sup \left(\left|u_{2}\right|^{1+\varepsilon}\left|u_{1}\right|,\left|u_{1}\right|^{1+\varepsilon}\left|u_{2}\right|\right) \geqslant(1-\varepsilon)^{1+2 \varepsilon}
$$

for every $\left(u_{1}, u_{2}\right) \in G_{\varepsilon}\left(\mathbf{C}^{2}\right)$.

Condition (5) shows that $G_{\varepsilon}\left(\mathbf{C}^{2}\right)$ avoids then a neighborhood of the set $\Delta=\left\{\left(z_{1}, z_{2}\right) \in \mathbf{C}^{2} \mid \pi_{1}(z) \cdot \pi_{2}(z)=0\right\}$, which is the union of two complex lines.

In a recent paper [77, Example 5], which reached the authors after this work was completed, Nishimura produces, for every $\alpha>0$, an entire mapping $H_{\alpha}: \mathbf{C}^{2} \rightarrow \mathrm{C}^{2}$, with nonvanishing jacobian, whose range avoids $\Delta \cup D(0, \alpha)$. On the one hand condition (5) above is stronger than Nishimura's condition. On the other hand if we take $\alpha=\varepsilon^{-1}$ then we obtain $\inf \left(\left|u_{1}\right|,\left|u_{2}\right|\right)<\varepsilon$ for every $\left(u_{1}, u_{2}\right) \in\left(\Psi \circ H_{\alpha}\right)\left(\mathbf{C}^{2}\right)$, where $H_{\alpha}$ is Nishimura's map and $\Psi$ is the map $\left(z_{1}, z_{2}\right) \rightarrow\left(1 / z_{1}, 1 / z_{2}\right)$. (Nishimura's construction also uses Arakelian's theorem.)

(3) Bloch's theorem shows that if $f: D(0,1) \rightarrow \mathrm{C}$ is an analytic function of one complex variable such that $f^{\prime}(0)=1$ then $f(D(0,1))$ contains an open disc of radius $K$, where $K$ is the Bloch constant, which does not depend on $f$ (see [27, Chapter 12, Definition 1.8]). Corollary 8.4 shows that this phenomenon does not hold for analytic functions of two complex variables. Consider for example the function $G_{1 / 2}$ given by Corollary 8.5 . For every $\eta>0$, there exists a linear map $L_{\eta}$ such that $\left(\eta\left(G_{1 / 2} \circ L_{\eta}\right)\right)^{\prime}(0)=I$, but $\left[\eta\left(G_{1 / 2} \circ L_{\eta}\right)\right]\left(\mathbf{C}^{2}\right)$ is contained in the union of two polycylinders of radius $\eta$, so that $\left[\eta\left(G_{1 / 2} \circ L_{\eta}\right)\right]\left(\mathbf{C}^{2}\right)$ does not contain any polydisc of radius greater than $\eta$.

(4) In $\$ 4$, we made the obvious observation that the volume of $F\left(\mathbf{C}^{p}\right)$ is infinite if $F: \mathbf{C}^{p} \rightarrow \mathbf{C}^{p}$ is a one-to-one entire function. On the other hand, a routine computation that we omit shows that $m\left(G_{\varepsilon}\left(\mathbf{C}^{2}\right) \cap D(0, r)\right)=O\left(r^{2 \varepsilon}\right)$ 
where $G_{\varepsilon}$ is the function given in Corollary 8.4. It seems very likely that the volume of $F\left(\mathbf{C}^{p}\right)$ is infinite for every nondegenerate entire function from $\mathbf{C}^{p}$ into itself, but the authors have not been able to prove this.

It is nevertheless possible to modify the function $G_{\varepsilon}$ given by Corollary 8.5 to arrange the volume of one of the branches of the image of $\mathbf{C}^{2}$ to have finite volume. For example take $\varepsilon=\frac{1}{2}$ and consider the function $H=\Psi \circ G_{1 / 2}$, where $\Psi$ is the function $\left(z_{1}, z_{2}\right) \rightarrow\left(z_{1}^{2}, z_{2}\right)$. If $\left(u_{1}, u_{2}\right) \in H\left(\mathbf{C}^{2}\right)$, with $\left|u_{2}\right| \geqslant 1$ then $\left(u_{1}, u_{2}\right)=\left(v^{2}, u_{2}\right)$ where $|v|^{3 / 2} \cdot\left|u_{2}\right| \leqslant 1$ so that $\left|u_{1}\right|^{3 / 4}\left|u_{2}\right| \leqslant 1$. So

$$
\begin{aligned}
m\left[H\left(\mathbf{C}^{2}\right) \cap\left\{z \in \mathbf{C}^{2}|| \pi_{2}(z) \mid \geqslant 1\right\}\right] & \leqslant 4 \pi^{2} \int_{0}^{1} t\left[\int_{0}^{t^{-3 / 4}} s d s\right] d t \\
& \leqslant 2 \pi^{2} \int_{0}^{1} t^{-1 / 2} d t<+\infty .
\end{aligned}
$$

We thus see that $H\left(\mathbf{C}^{2}\right)=U_{1} \cup U_{2}$ where $U_{1}=\left\{z \in H\left(\mathbf{C}^{2}\right)|| \pi_{1}(z) \mid \leqslant 1\right\}$, $U_{2}=\left\{z \in H\left(\mathbf{C}^{2}\right)|| \pi_{2}(z) \mid \leqslant 1\right\}$, and where $m\left(U_{1}\right)<+\infty$. Since $\inf \left(\left|\pi_{1}(z)\right|\right.$, $\left.\left|\pi_{2}(z)\right|\right) \underset{|z| \rightarrow \infty}{\rightarrow} 0, z \in H\left(\mathbf{C}^{2}\right)$, we even have

$$
m\left(\left\{z \in H\left(\mathbf{C}^{2}\right)|| \pi_{2}(z) \mid>\eta\right\}\right)<+\infty
$$

for every $\eta>0$.

Now we wish to produce a Bieberbach function $F$ whose range avoids $U_{1}$ but contains $-U_{1}$. In order to get some qualitative information about the inverse images of bounded sets for $F$, we give an application of Lemma 8.1 which is more precise than the one given in Lemma 8.2. We set $\rho(z)=$ $\inf \left(\left|\pi_{1}(z)\right|,\left|\pi_{2}(z)\right|\right)$ for $z \in \mathbf{C}^{2}$.

Lemma 8.7. Let $a, b, \lambda, \mu$ be four real numbers, and let $\delta>0, \eta>0$. There exists $\Theta \in$ Aut $_{1}\left(\mathbf{C}^{2}\right)$ which possesses the following properties.

$$
\begin{aligned}
& |\Theta(z)-z+(\lambda, \mu)|<\eta e^{-\rho(z)^{1 / 4}} \\
& \text { for } \operatorname{Re} \pi_{1}(z) \geqslant a+\delta, \operatorname{Re} \pi_{2}(z) \geqslant b+\delta . \\
& |\Theta(z)-z+(\lambda, 0)|<\eta e^{-\rho(z)^{1 / 4}} \\
& \text { for } \operatorname{Re} \pi_{1}(z) \leqslant a-\delta, \operatorname{Re} \pi_{2}(z) \geqslant b-\mu+\delta . \\
& |\Theta(z)-z+(0, \mu)|<\eta e^{-\rho(z)^{1 / 4}} \\
& \text { for } \operatorname{Re} \pi_{1}(z) \geqslant a+\delta, \operatorname{Re} \pi_{2}(z) \leqslant b-\delta .
\end{aligned}
$$

Proof. We may assume without loss of generality that $\eta=\delta$. It follows from Lemma 8.1 that there exist a sequence $\left(f_{p}\right)$ of entire functions over $\mathbf{C}$ such that $\left|f_{p}(\xi)\right|<e^{-|\xi|^{1 / 4}} / p \quad(p \geqslant 1, \operatorname{Re} \xi \leqslant b-\mu-\delta / 2), \quad\left|f_{p}(\xi)-\lambda\right|<$ $e^{-|\xi|^{1 / 4}} / p(p \geqslant 1, \operatorname{Re} \xi \geqslant b-\mu+\delta / 2)$ and a sequence $\left(g_{p}\right)$ of entire functions over $\mathbf{C}$ such that $\left|g_{p}(\xi)\right|<e^{-|\xi|^{1 / 4}} / p(p \geqslant 1, \operatorname{Re} \xi \leqslant a-\delta / 2),\left|g_{p}(\xi)-\mu\right|<$ $e^{-|\xi|^{1 / 4}} / p(p \geqslant 1, \operatorname{Re} \xi \geqslant a+\delta / 2)$. Denote by $\Theta_{p}$ the map $\left(z_{1}, z_{2}\right) \rightarrow\left(v_{1}, v_{2}\right)$ where $v_{2}=z_{2}-g_{p}\left(z_{1}\right), v_{1}=z_{1}-f_{p}\left(v_{2}\right)$. If $\operatorname{Re} z_{1} \geqslant a+\delta / 2$ then

$$
\left|v_{2}-z_{2}+\mu\right|=\left|g_{p}\left(z_{1}\right)-\mu\right|<e^{-\left|z_{1}\right|^{1 / 4}} / p \leqslant e^{-\rho(z)^{1 / 4}} / p,
$$

and if $\operatorname{Re} z_{1} \leqslant a-\delta / 2$ then

$$
\left|v_{2}-z_{2}\right|=\left|g_{p}\left(z_{1}\right)\right| \leqslant e^{-\rho(z)^{1 / 4}} / p .
$$


So if $p \geqslant 2 / \delta, \operatorname{Re} z_{1} \geqslant a+\delta / 2, \operatorname{Re} z_{2} \geqslant b+\delta$ then $\operatorname{Re} v_{2} \geqslant b-\mu+\delta / 2$, $\left|f_{p}\left(v_{2}\right)-\lambda\right|<e^{-\left|v_{2}\right|^{1 / 4}} / p$, hence $\left|v_{1}-z_{1}+\lambda\right| \leqslant e^{-\left|v_{2}\right|^{1 / 4}} / p$. Since $\left|v_{2}\right|>\left|z_{2}\right|-$ $|\mu|-1$ and since $t^{1 / 4}-(t-|\mu|-1)^{1 / 4} \underset{t \rightarrow \infty}{\rightarrow} 0$, we obtain

$$
\left|\Theta_{p}(z)-z+(\lambda, \mu)\right| \leqslant k e^{-\rho(z)^{1 / 4}} / p
$$

where $k \geqslant 1$ is some constant. So condition (1) is satisfied by $\Theta_{p}$ when $p>2 k / \delta$. Similar considerations show in fact that the four conditions are satisfied by $\Theta_{p}$ when $p$ is sufficiently large. This proves the lemma.

The following lemma will enable us to avoid some tedious computations in the proof of the next theorem.

LEMMA 8.8. Let $r>0$ and let $F: \overline{D((0,0), r)} \rightarrow \mathrm{C}^{2}$ be a continuous map. If $|F(z)-z|<r$ for every $z \in \overline{D((0,0), r)}$, then there exists $u \in D((0,0), r)$ such that $F(u)=(0,0)$.

Proof. Since $\overline{D((0,0), r)}$ is compact and convex, this follows from the Brouwer-Schauder-Tychonoff fixed point theorem [39, Theorem 5.10.5] applied to the map $z \rightarrow z-F(z)$ of $\overline{D((0,0), r)}$ into itself. (Note that for analytic maps the result follows easily from Rouche's theorem.)

TheOREM 8.9. Let $\varepsilon \in(0,1 / 2)$. Set

$$
\begin{aligned}
A= & \left\{z \in \mathbf{C}^{2} \mid \operatorname{Re} \pi_{2}(z)=0\right\} \cup\left\{z \in \mathbf{C}^{2} \mid \operatorname{Re} \pi_{1}(z)=0, \operatorname{Re} \pi_{2}(z) \geqslant 0\right\} \\
& \cup\left\{z \in \mathbf{C}^{2} \mid \operatorname{Re} \pi_{1}(z) \leqslant 0, \operatorname{Re} \pi_{2}(z) \in \mathbf{N}\right\} \\
& \cup\left\{z \in \mathbf{C}^{2} \mid \operatorname{Re} \pi_{2}(z) \leqslant 0, \operatorname{Re} \pi_{1}(z) \in \mathbf{N}\right\} .
\end{aligned}
$$

Set

$$
\begin{gathered}
B=\left\{z \in \mathbf{C}^{2} \mid \operatorname{Re} \pi_{2}(z)+1 \in \mathbf{N}, \operatorname{Re} \pi_{1}(z) \leqslant \operatorname{Re} \pi_{2}(z)+1\right\} \\
\cup\left\{z \in \mathbf{C}^{2} \mid \operatorname{Re} \pi_{1}(z) \in \mathbf{N}, \operatorname{Re} \pi_{2}(z) \leqslant \operatorname{Re} \pi_{1}(z)\right\}
\end{gathered}
$$

Set

$$
\begin{aligned}
W_{\varepsilon}= & \left(\bigcup_{k \geqslant 1}\left\{z \in \mathbf{C}^{2}|| \operatorname{Re} \pi_{2}(z)-k+1 \mid<\varepsilon / 2^{k}, \operatorname{Re} \pi_{1}(z) \leqslant k\right\}\right) \\
& \cup\left(\bigcup_{k \geqslant 1}\left\{z \in \mathbf{C}^{2}|| \operatorname{Re} \pi_{1}(z)-k \mid<\varepsilon / 2^{k}, \operatorname{Re} \pi_{2}(z) \leqslant k\right\}\right) \\
& \cup\left(\bigcup_{k \geqslant 1}\left\{z \in \mathbf{C}^{2} \mid k-\varepsilon / 2<\operatorname{Re} \pi_{2}(z) \leqslant k, k \leqslant \operatorname{Re} \pi_{1}(z) \leqslant k+1\right\}\right) \\
& \cup\left(\bigcup_{k \geqslant 1}\left\{z \in \mathbf{C}^{2} \mid k-\varepsilon / 2<\operatorname{Re} \pi_{1}(z) \leqslant k, k-1 \leqslant \operatorname{Re} \pi_{2}(z) \leqslant k\right\}\right) .
\end{aligned}
$$

Set $\Omega=\left\{z \in \mathbf{C}^{2} \backslash A \mid \inf \left(\operatorname{Re} \pi_{1}(z), \operatorname{Re} \pi_{2}(z)\right) \leqslant 0\right\}$. 
Let $S: \mathbf{C}^{2} \backslash B \rightarrow \mathbf{C}^{2}$ and $T: \Omega \rightarrow \mathbf{C}^{2}$ be the maps defined by the formulae

$$
\begin{array}{ll}
S(z)=T(z)=z & \left(\operatorname{Re} \pi_{1}(z)<1, \operatorname{Re} \pi_{2}(z)<0\right) \\
S(z)=z-(k, 0) & \left(\operatorname{Re} \pi_{1}(z)<k, k-1<\operatorname{Re} \pi_{2}(z)<k, k \geqslant 1\right) \\
S(z)=z-(0, k) & \left(k<\operatorname{Re} \pi_{1}(z)<k+1, \operatorname{Re} \pi_{2}(z)<k, k \geqslant 1\right) \\
T(z)=z+(k, 0) & \left(\operatorname{Re} \pi_{1}(z)<0, k-1<\operatorname{Re} \pi_{2}(z)<k, k \geqslant 1\right) \\
T(z)=z+(0, k) & \left(k<\operatorname{Re} \pi_{1}(z)<k+1, \operatorname{Re} \pi_{2}(z)<0, k \geqslant 1\right) .
\end{array}
$$

Then there exists a Bieberbach function $F: \mathbf{C}^{2} \rightarrow \mathbf{C}^{2}$, which possesses the following properties.

(1) If $\operatorname{Re} \pi_{1}(z) \geqslant \varepsilon, \operatorname{Re} \pi_{2}(z) \geqslant \varepsilon$, then $z \notin F\left(\mathbf{C}^{2}\right)$.

(2) If $z \in \Omega$, and if $d(z, A) \geqslant \varepsilon$, then $z \in F\left(\mathbf{C}^{2}\right)$, and $\left|F^{-1}(z)-T(z)\right|<\varepsilon$.

(3) If $z \in \mathbf{C}^{2} \backslash W_{\varepsilon}$, then $|F(z)-S(z)|<\varepsilon$.

(4) If $d(z, A)<\varepsilon$, and if $z \in F\left(\mathbf{C}^{2}\right)$, then $d\left(F^{-1}(z), B\right)<2 \varepsilon$.

(5) If $d(z, B)<\varepsilon$, then $d(F(z), A)<2 \varepsilon$.

(6) $F^{-1}(\Delta) \subset W_{\varepsilon} \cup T\left(\Delta_{\varepsilon} \cap \Omega\right)$, where $\Delta_{\varepsilon}=\left\{z \in \mathbf{C}^{2} \mid d(z, \Delta)<\varepsilon\right\}$, for every subset $\Delta$ of $\mathbf{C}^{2}$.

(7) If $\Delta$ is a bounded subset of $\mathbf{C}^{2}$, then the set

$$
\left\{z \in F^{-1}(\Delta) \mid \max \left(\operatorname{Re} \pi_{1}(z), \operatorname{Re} \pi_{2}(z)\right) \leqslant a\right\}
$$

is bounded for every $a \in \mathbf{R}$.

Proof. Notice that $S\left(\mathbf{C}^{2} \backslash B\right)=\Omega, T(\Omega)=\mathbf{C}^{2} \backslash B$ and that $T=S^{-1}$. Assume that an entire mapping $F: \mathbf{C}^{2} \rightarrow \mathbf{C}^{2}$ satisfies (1), (2), (3). If $u \in F\left(\mathbf{C}^{2}\right)$, $d(u, A) \geqslant 2 \varepsilon$, then it follows from (1) and (2) that $d\left(F^{-1}(u), B\right)>\varepsilon$. So if $d(z, B) \leqslant \varepsilon$, then $d(F(z), A)<2 \varepsilon$. Also if $d(u, B) \geqslant 2 \varepsilon$ then it follows from (3) that $d(F(u), A)>\varepsilon$. So if $z \in F\left(\mathbf{C}^{2}\right)$, and if $d(z, A) \leqslant \varepsilon$, then $d\left(F^{-1}(z), B\right)<2 \varepsilon$. This shows that $F$ satisfies (4) and (5).

Now let $\Delta$ be a subset of $\mathbf{C}^{2}$. If $z \in F^{-1}(\Delta), z \notin W_{\varepsilon}$, then $|S(z)-F(z)|<\varepsilon$. Hence $S(z) \in \Delta_{\varepsilon}$. Since $S(z) \in \Omega$ we obtain $z=T(S(z)) \in T\left(\Delta_{\varepsilon} \cap \Omega\right)$. Hence $F^{-1}(\Delta) \subset W_{\varepsilon} \cup T\left(\Delta_{\varepsilon} \cap \Omega\right)$, and so $F$ satisfies (6).

It follows easily from Lemma 8.8 that if $F$ is a one-to-one entire mapping which satisfies $|F(z)-S(z)|<\varepsilon / 2$ for $z \in \mathbf{C}^{2} \backslash W_{\varepsilon}$, then $F$ satisfies (2), but it does not seem that this stronger version of (3) is sufficient to obtain (1) directly.

We now construct the required function $F$. For $n \geqslant 1$ let $\Theta_{n}$ be an element of $\operatorname{Aut}_{1}\left(\mathbf{C}^{2}\right)$ which satisfies the conditions of Lemma 8.7 with $\lambda=\mu=1$, $a=b=n, \delta=\eta=\varepsilon / 2^{n+1}$.

It follows from condition (4) of Lemma 8.7 that

$$
\sum_{n=m+2}^{\infty}\left\|I-\Theta_{n}\right\|_{m}<\frac{\varepsilon}{2^{m+2}} \quad(m \geqslant 1)
$$

It follows then from Lemma 8.3 that the sequence $\left(F_{n}\right)_{n \geqslant 1}=$ $\left(\Theta_{1} \circ \ldots \circ \Theta_{n}\right)_{n \geqslant 1}$ converges uniformly over compact subsets of $\mathbf{C}^{2}$ to a one-to-one entire mapping $F: \mathrm{C}^{2} \rightarrow \mathrm{C}^{2}$. 
For $n \geqslant 1$ set

$$
\begin{aligned}
A_{n}= & \left\{z \in A \mid \operatorname{Re} \pi_{1}(z) \leqslant n, \operatorname{Re} \pi_{2}(z) \leqslant n-1\right\} \\
& \cup\left\{z \in \mathbf{C}^{2} \mid \operatorname{Re} \pi_{1}(z)=0, \operatorname{Re} \pi_{2}(z) \geqslant n-1\right\} \\
& \cup\left\{z \in \mathbf{C}^{2} \mid \operatorname{Re} \pi_{1}(z) \geqslant n, \operatorname{Re} \pi_{2}(z)=0\right\},
\end{aligned}
$$

and set

$$
\begin{aligned}
B_{n}= & \left\{z \in B \mid \operatorname{Re} \pi_{1}(z) \leqslant n, \operatorname{Re} \pi_{2}(z) \leqslant n-1\right\} \\
& \cup\left\{z \in \mathbf{C}^{2} \mid \operatorname{Re} \pi_{1}(z)=n, \operatorname{Re} \pi_{2}(z) \geqslant n-1\right\} \\
& \cup\left\{z \in \mathbf{C}^{2} \mid \operatorname{Re} \pi_{1}(z) \geqslant n, \operatorname{Re} \pi_{2}(z)=n\right\} .
\end{aligned}
$$

We define $S_{n}: \mathbf{C}^{2} \backslash B_{n} \rightarrow \mathbf{C}^{2}$ and $T_{n}: \mathbf{C}^{2} \backslash A_{n} \rightarrow \mathbf{C}^{2}$ by the formulae

$$
\begin{array}{ll}
S_{n}(z)=z-(n, n) & \text { if } \operatorname{Re} \pi_{1}(z)>n, \operatorname{Re} \pi_{2}(z)>n, \\
S_{n}(z)=z-(n, 0) & \text { if } \operatorname{Re} \pi_{1}(z)<n, \operatorname{Re} \pi_{2}(z)>n-1, \\
S_{n}(z)=z-(0, n) & \text { if } \operatorname{Re} \pi_{1}(z)>n, \operatorname{Re} \pi_{2}(z)<n, \\
S_{n}(z)=S(z) & \text { if } \operatorname{Re} \pi_{1}(z)<n, \operatorname{Re} \pi_{2}(z)<n-1, \\
T_{n}(z)=z+(n, n) & \text { if } \operatorname{Re} \pi_{1}(z)>0, \operatorname{Re} \pi_{2}(z)>0, \\
T_{n}(z)=z+(n, 0) & \text { if } \operatorname{Re} \pi_{1}(z)<0, \operatorname{Re} \pi_{2}(z)>n-1, \\
T_{n}(z)=z+(0, n) & \text { if } \operatorname{Re} \pi_{1}(z)>n, \operatorname{Re} \pi_{2}(z)<0, \\
T_{n}(z)=T(z) & \text { if } \operatorname{Re} \pi_{1}(z)<0, \operatorname{Re} \pi_{2}(z)<n-1 \text { or } \\
& \text { if } \operatorname{Re} \pi_{1}(z)<n, \operatorname{Re} \pi_{2}(z)<0 .
\end{array}
$$

We have $S_{n}\left(\mathbf{C}^{2} \backslash B_{n}\right)=\mathbf{C}^{2} \backslash A_{n}, T_{n}\left(\mathbf{C}^{2} \backslash A_{n}\right)=\mathbf{C}^{2} \backslash B_{n}$ and $T_{n}=S_{n}^{-1}(n \geqslant 1)$. Denote by $\Delta_{n}$ the set of all elements $z$ of $C^{2}$ which satisfy one of the following conditions

$\left(a_{n}\right) \quad \operatorname{Re} \pi_{1}(z) \geqslant n+\frac{\varepsilon}{2}-\frac{\varepsilon}{2^{n+1}}, \quad \operatorname{Re} \pi_{2}(z) \geqslant n+\frac{\varepsilon}{2}-\frac{\varepsilon}{2^{n+1}}$.

$\left(b_{n}\right) \quad \operatorname{Re} \pi_{1}(z) \leqslant n-\frac{\varepsilon}{2}+\frac{\varepsilon}{2^{n+1}}, \quad \operatorname{Re} \pi_{2}(z) \geqslant n-1+\frac{\varepsilon}{2^{n+1}}$.

$\left(c_{n}\right) \quad \operatorname{Re} \pi_{1}(z) \geqslant n+\frac{\varepsilon}{2^{n+1}}, \quad \operatorname{Re} \pi_{2}(z) \leqslant n-\frac{\varepsilon}{2}+\frac{\varepsilon}{2^{n+1}}$.

$\left(d_{n}\right) \quad \operatorname{Re} \pi_{1}(z) \leqslant 1-\frac{\varepsilon}{2}+\frac{\varepsilon}{2^{n+1}}, \quad \operatorname{Re} \pi_{2}(z) \leqslant-\frac{\varepsilon}{2}+\frac{\varepsilon}{2^{n+1}}$.

$\left(e_{n}\right) \quad \operatorname{Re} \pi_{1}(z) \leqslant k-\frac{\varepsilon}{2}+\frac{\varepsilon}{2^{n+1}}$,

$$
k-1+\frac{\varepsilon}{2^{k}}-\frac{\varepsilon}{2^{n+1}} \leqslant \operatorname{Re} \pi_{2}(z) \leqslant k-\frac{\varepsilon}{2^{k+1}}+\frac{\varepsilon}{2^{n+1}}
$$

for some $k \in\{1, \ldots, n-1\}$.

$\left(f_{n}\right) \quad k+\frac{\varepsilon}{2^{k}}-\frac{\varepsilon}{2^{n+1}} \leqslant \operatorname{Re} \pi_{1}(z) \leqslant k+1-\frac{\varepsilon}{2^{k+1}}+\frac{\varepsilon}{2^{n+1}}$,

$$
\operatorname{Re} \pi_{2}(z) \leqslant k-\frac{\varepsilon}{2}+\frac{\varepsilon}{2^{n+1}} \quad \text { for some } k \in\{1, \ldots, n-1\} .
$$


We have the following property:

$$
\left|F_{n}(z)-S_{n}(z)\right|<\frac{\varepsilon}{2}-\frac{\varepsilon}{2^{n+1}} \quad\left(z \in \Delta_{n}, n \geqslant 1\right) .
$$

It follows from Lemma 8.7 that (8) holds for $n=1$. Assume that (8) holds for some $n \geqslant 1$. If $z$ satisfies $\left(a_{n+1}\right)$ then it follows from Lemma 8.7 that $\left|\Theta_{n+1}(z)-z+(1,1)\right|<\varepsilon / 2^{n+2}$, so that $z$ satisfies $\left(a_{n}\right)$. It follows then from the induction hypothesis that

$$
\left|F_{n+1}(z)-\Theta_{n+1}(z)+(n, n)\right|<\frac{\varepsilon}{2}-\frac{\varepsilon}{2^{n+1}},
$$

and so

$$
\left|F_{n+1}(z)-S_{n+1}(z)\right|=\left|F_{n+1}(z)-z+(n+1, n+1)\right|<\frac{\varepsilon}{2}-\frac{\varepsilon}{2^{n+2}} .
$$

If $z$ satisfies $\left(b_{n+1}\right)$ then it follows from Lemma 8.7 that $\Theta_{n+1}(z)-z+$ $(1,0) \mid<\varepsilon / 2^{n+2}$. So $\Theta_{n+1}(z)$ satisfies $\left(b_{n}\right)$ and it follows from the induction hypothesis that

$$
\left|F_{n+1}(z)-\Theta_{n+1}(z)+(n, 0)\right|<\frac{\varepsilon}{2}-\frac{\varepsilon}{2^{n+1}} .
$$

Hence

$$
\left|F_{n+1}(z)-S_{n+1}(z)\right|=\left|F_{n+1}(z)-z+(n+1,0)\right|<\frac{\varepsilon}{2}-\frac{\varepsilon}{2^{n+2}} .
$$

If $z$ satisfies $\left(c_{n+1}\right)$ then it follows from Lemma 8.7 that $\Theta_{n+1}(z)-z+$ $(0,1) \mid<\varepsilon / 2^{n+2}$. So $\Theta_{n+1}(z)$ satisfies $\left(c_{n}\right)$, and it follows from the induction hypothesis that $\left|F_{n+1}(z)-\Theta_{n+1}(z)+(0, n)\right|<\varepsilon / 2-\varepsilon / 2^{n+1}$. Hence

$$
\left|F_{n+1}(z)-S_{n+1}(z)\right|=\left|F_{n+1}(z)-z+(0, n+1)\right|<\varepsilon / 2-\varepsilon / 2^{n+2} .
$$

If $z$ satisfies $\left(d_{n+1}\right)$, or if $z$ satisfies $\left(e_{n+1}\right)$ for some $k \in\{1, \ldots, n\}$, or if $z$ satisfies $\left(f_{n+1}\right)$ for some $k \in\{1, \ldots, n\}$, then

$$
\begin{aligned}
& \operatorname{Re} \pi_{1}(z) \leqslant n+1-\frac{\varepsilon}{2^{n+1}}+\frac{\varepsilon}{2^{n+2}}=n+1-\frac{\varepsilon}{2^{n+2}}, \\
& \operatorname{Re} \pi_{2}(z) \leqslant n-\frac{\varepsilon}{2^{n+1}}+\frac{\varepsilon}{2^{n+2}}=n-\frac{\varepsilon}{2^{n+2}}
\end{aligned}
$$

and so it follows from Lemma 8.7 that $\left|\Theta_{n+1}(z)-z\right|<\varepsilon / 2^{n+2}$.

If $z$ satisfies $\left(d_{n+1}\right)$, then $\Theta_{n+1}(z)$ satisfies $\left(d_{n}\right)$, so that $\left|F_{n+1}(z)-\Theta_{n+1}(z)\right|$ $<\varepsilon / 2-\varepsilon / 2^{n+1}$, and

$$
\left|F_{n+1}(z)-S_{n+1}(z)\right|=\left|F_{n+1}(z)-z\right|<\frac{\varepsilon}{2}-\frac{\varepsilon}{2^{n+2}} .
$$

If $z$ satisfies $\left(e_{n+1}\right)$ for some $k \in\{1, \ldots, n-1\}$, then $\Theta_{n+1}(z)$ satisfies $\left(e_{n}\right)$ for $k$, so that $\left|F_{n+1}(z)-\Theta_{n+1}(z)+(k, 0)\right|<\varepsilon / 2-\varepsilon / 2^{n+1}$. Hence

$$
\left|F_{n+1}(z)-S_{n+1}(z)\right|=\left|F_{n+1}(z)-z+(k, 0)\right|<\frac{\varepsilon}{2}-\frac{\varepsilon}{2^{n+2}} .
$$

If $z$ satisfies $\left(f_{n+1}\right)$ for some $k \in\{1, \ldots, n-1\}$, then $\Theta_{n+1}(z)$ satisfies $\left(f_{n}\right)$ for $k$, so that $\left|F_{n+1}(z)-\Theta_{n+1}(z)+(0, k)\right|<\varepsilon / 2-\varepsilon / 2^{n+1}$. Hence

$$
\left|F_{n+1}(z)-S_{n+1}(z)\right|=\left|F_{n+1}(z)-z+(0, k)\right|<\frac{\varepsilon}{2}-\frac{\varepsilon}{2^{n+2}} .
$$


If $z$ satisfies $\left(e_{n+1}\right)$ for $k=n$, then $\Theta_{n+1}(z)$ satisfies $\left(b_{n}\right)$. Hence $\mid F_{n+1}(z)-$ $\Theta_{n+1}(z)+(n, 0) \mid<\varepsilon / 2-\varepsilon / 2^{n+1}$, and so

$$
\left|F_{n+1}(z)-S_{n+1}(z)\right|=\left|F_{n+1}(z)-z+(n, 0)\right|<\frac{\varepsilon}{2}-\frac{\varepsilon}{2^{n+2}} .
$$

If $z$ satisfies $\left(f_{n+1}\right)$ for $k=n$, then $\Theta_{n+1}(z)$ satisfies $\left(c_{n}\right)$. Hence $\mid F_{n+1}(z)-$ $\Theta_{n+1}(z)+(0, n) \mid<\varepsilon / 2-\varepsilon / 2^{n+1}$, and so

$$
\left|F_{n+1}(z)-S_{n+1}(z)\right|=\left|F_{n+1}(z)-z+(0, n)\right|<\frac{\varepsilon}{2}-\frac{\varepsilon}{2^{n+2}} .
$$

So the inequality $\left|F_{n+1}(z)-S_{n+1}(z)\right|<\varepsilon / 2-\varepsilon / 2^{n+2}$ holds for every $z \in$ $\Delta_{n+1}$. We obtain (8) by induction.

Now let $z \in \mathbf{C}^{2}$ such that $d\left(z, A_{n}\right) \geqslant \varepsilon-\varepsilon / 2^{n}$. Then $d\left(T_{n}(z), B_{n}\right) \geqslant \varepsilon-$ $\varepsilon / 2^{n}$, and so $T_{n}(z)+u \in \Delta_{n}$ if $|u| \leqslant \varepsilon / 2-\varepsilon / 2^{n+1}$.

Let $K$ be the component of $\mathbf{C}^{2} \backslash B_{n}$ which contains $T_{n}(z)$. There exists $a \in \mathbf{C}^{2}$ which is such that $S_{n}(u)=u-a$ for every $u \in K$.

Set $G_{n}(u)=F_{n}\left(T_{n}(z)+u\right)-z \quad\left(u \in \mathbf{C}^{2}\right)$. If $|u| \leqslant \varepsilon / 2-\varepsilon / 2^{n+1}$, then $T_{n}(z)+u \in \Delta_{n} \cap K$ and so $S_{n}\left(T_{n}(z)+u\right)=T_{n}(z)+u-a=S_{n}\left(T_{n}(z)\right)+u$ $=z+u$. Hence

$$
\begin{aligned}
\left|G_{n}(u)-u\right| & =\left|F_{n}\left(T_{n}(z)+u\right)-z-u\right| \\
& =\left|F_{n}\left(T_{n}(z)+u\right)-S_{n}\left(T_{n}(z)+u\right)\right|<\frac{\varepsilon}{2}-\frac{\varepsilon}{2^{n+1}} .
\end{aligned}
$$

It follows then from Lemma 8.8 that there exists $u \in D\left(0, \varepsilon / 2-\varepsilon / 2^{n+1}\right)$ which satisfies $G_{n}(u)=0$. We obtain $F_{n}\left(T_{n}(z)+u\right)=z, T_{n}(z)+u=F_{n}^{-1}(z)$ and so $\left|F_{n}^{-1}(z)-T_{n}(z)\right|=|u|<\varepsilon / 2-\varepsilon / 2^{n+1}$. So we have the inequality

$$
\left|F_{n}^{-1}(z)-T_{n}(z)\right|<\frac{\varepsilon}{2}-\frac{\varepsilon}{2^{n+1}} \quad\left(d\left(z, A_{n}\right) \geqslant \varepsilon-\frac{\varepsilon}{2^{n}}, n \geqslant 1\right) .
$$

If $z \in \mathbf{C}^{2} \backslash W_{\varepsilon}$, then $z \in \Delta_{n}$ when $n$ is sufficiently large. It follows then from (8) that $\left|F_{n}(z)-S(z)\right|=\left|F_{n}(z)-S_{n}(z)\right|<\varepsilon / 2-\varepsilon / 2^{n+1}$ when $n$ is sufficiently large. So $F$ satisfies condition (3) of the theorem.

If $\operatorname{Re} \pi_{1}(z) \geqslant \varepsilon, \operatorname{Re} \pi_{2}(z) \geqslant \varepsilon$ then $d\left(z, A_{n}\right) \geqslant \varepsilon$ and so it follows from (9) that $\left|F_{n}^{-1}(z)-(n, n)\right|=\left|F_{n}^{-1}(z)-T_{n}(z)\right|<\varepsilon / 2-\varepsilon / 2^{n+1}$ for every $n \geqslant 1$. Hence $\liminf _{n \rightarrow \infty}\left|F_{n}^{-1}(z)\right|=+\infty$, and so it follows from Corollary 5.3 that $z \notin F\left(\mathbf{C}^{2}\right)$. So $F$ satisfies condition (1) of the theorem.

Now if $z \in \Omega$ and if $d(z, A) \geqslant \varepsilon$ then $d\left(z, A_{n}\right) \geqslant \varepsilon$, and so it follows from (9) that $\left|F_{n}^{-1}(z)-T_{n}(z)\right|<\varepsilon / 2-\varepsilon / 2^{n+1}$ for every $n \geqslant 1$. Since $T_{n}(z)=T(z)$ when $n$ is sufficiently large it follows then from Corollary 5.3 that $F$ satisfies condition (2) of the theorem.

Notice that it follows from (8) that we have the following property, for every subset $\Delta$ of $\mathbf{C}^{2}$ :

$$
F_{n}^{-1}(\Delta) \subset\left(\mathbf{C}^{2} \backslash \Delta_{n}\right) \cup T_{n}\left(\left\{z \in \mathbf{C}^{2} \backslash A_{n} \mid d(z, \Delta)<\frac{\varepsilon}{2}-\frac{\varepsilon}{2^{n+1}}\right\}\right) \quad(n \geqslant 1) .
$$


It remains to prove that $F$ satisfies condition (7) of the theorem. To do this we shall use the following inequality.

$$
\left|\left(\Theta_{k} \circ \cdots \circ \Theta_{n}\right)(z)-z\right|<\frac{\varepsilon}{2^{k}}-\frac{\varepsilon}{2^{n+1}}
$$

$\left(\operatorname{Re} \pi_{1}(z) \leqslant k-\frac{\varepsilon}{2^{k}}+\frac{\varepsilon}{2^{n+1}}, \operatorname{Re} \pi_{2}(z) \leqslant k-1-\frac{\varepsilon}{2^{k}}+\frac{\varepsilon}{2^{n+1}}, n \geqslant k \geqslant 1\right)$.

It follows from Lemma 8.7 that (11) holds for $n=k, k \geqslant 1$. Now fix $k \geqslant 1$ and assume that (11) holds for some $n \geqslant k$. If

$$
\operatorname{Re} \pi_{1}(z) \leqslant k-\frac{\varepsilon}{2^{k}}+\frac{\varepsilon}{2^{n+2}}, \quad \operatorname{Re} \pi_{2}(z) \leqslant k-1-\frac{\varepsilon}{2^{k}}+\frac{\varepsilon}{2^{n+2}},
$$

then it follows from Lemma 8.7 that $\left|\Theta_{n+1}(z)-z\right|<\varepsilon / 2^{n+2}$. So $\operatorname{Re}\left(\pi_{1} \circ \Theta_{n+1}\right)(z) \leqslant k-\varepsilon / 2^{k}+\varepsilon / 2^{n+1}, \quad \operatorname{Re}\left(\pi_{2} \circ \Theta_{n+1}\right)(z) \leqslant k-1-\varepsilon / 2^{k}+$ $\varepsilon / 2^{n+1}$, and so it follows from the induction hypothesis that

$$
\left|\left(\Theta_{k} \circ \cdots \circ \Theta_{n+1}\right)(z)-\Theta_{n+1}(z)\right|<\frac{\varepsilon}{2^{k}}-\frac{\varepsilon}{2^{n+1}} .
$$

So $\left|\left(\Theta_{k} \circ \cdots \circ \Theta_{n+1}\right)(z)-z\right|<\varepsilon / 2^{k}-\varepsilon / 2^{n+2}$, and we obtain (11) by induction.

Notice that, by using Lemma 8.8 as in the proof of (9), we easily deduce from (11) the inequality

$$
\begin{aligned}
& \left|\left(\Theta_{n}^{-1} \circ \cdots \circ \Theta_{k}^{-1}\right)(z)-z\right|<\frac{\varepsilon}{2^{k}}-\frac{\varepsilon}{2^{n+1}} \\
& \left(\operatorname{Re} \pi_{1}(z) \leqslant k-\frac{\varepsilon}{2^{k-1}}+\frac{\varepsilon}{2^{n}},\right. \\
& \left.\quad \operatorname{Re} \pi_{2}(z) \leqslant k-1-\frac{\varepsilon}{2^{k-1}}+\frac{\varepsilon}{2^{n+1}}, n \geqslant k \geqslant 1\right) .
\end{aligned}
$$

Inequality (12) has some interest in itself, because it follows from (12) that if $\operatorname{Re}\left(\pi_{1} \circ F_{k}^{-1}\right)(z) \leqslant k+1-\varepsilon / 2^{k}, \quad \operatorname{Re}\left(\pi_{2} \circ F_{k}^{-1}\right)(z) \leqslant k-\varepsilon / 2^{k}$ then $z \in$ $F\left(\mathbf{C}^{2}\right)$, and $\left|F^{-1}(z)-F_{k}^{-1}(z)\right|<\varepsilon / 2^{k}$.

Now let $\Delta$ be a bounded subset of $\mathbf{C}^{2}$. If $z \in F^{-1}(\Delta)$ and if $\operatorname{Re} \pi_{1}(z) \leqslant k+$ $1-\varepsilon / 2^{k+1}, \operatorname{Re} \pi_{2}(z) \leqslant k-\varepsilon / 2^{k+1}$, where $k \geqslant 1$, then it follows from (11) that

$$
\left|z-\left(F_{k}^{-1} \circ F\right)(z)\right| \leqslant \limsup _{n \rightarrow \infty}\left|z-\left(\Theta_{k+1} \circ \cdots \circ \Theta_{n}\right)(z)\right| \leqslant \frac{\varepsilon}{2^{k+1}},
$$

and so $d\left(z, F_{k}^{-1}(\Delta)\right) \leqslant \varepsilon / 2^{k+1}$. Since $F_{k}^{-1}(\Delta)$ is bounded for every $k \geqslant 1, F$ satisfies condition (7), and the theorem is proved. (Conditions (1), (2), (3), (10), and (6) are illustrated in Figures 1, 2, 3, and 4.)

The role played by Lemma 8.8 in the above proof is purely technical. Information about the behavior of $\Theta^{-1}$ could be obtained directly in Lemma 8.7 to avoid the use of Lemma 8.8 in the proof of (9), but such arguments would necessitate more tedious computations. 


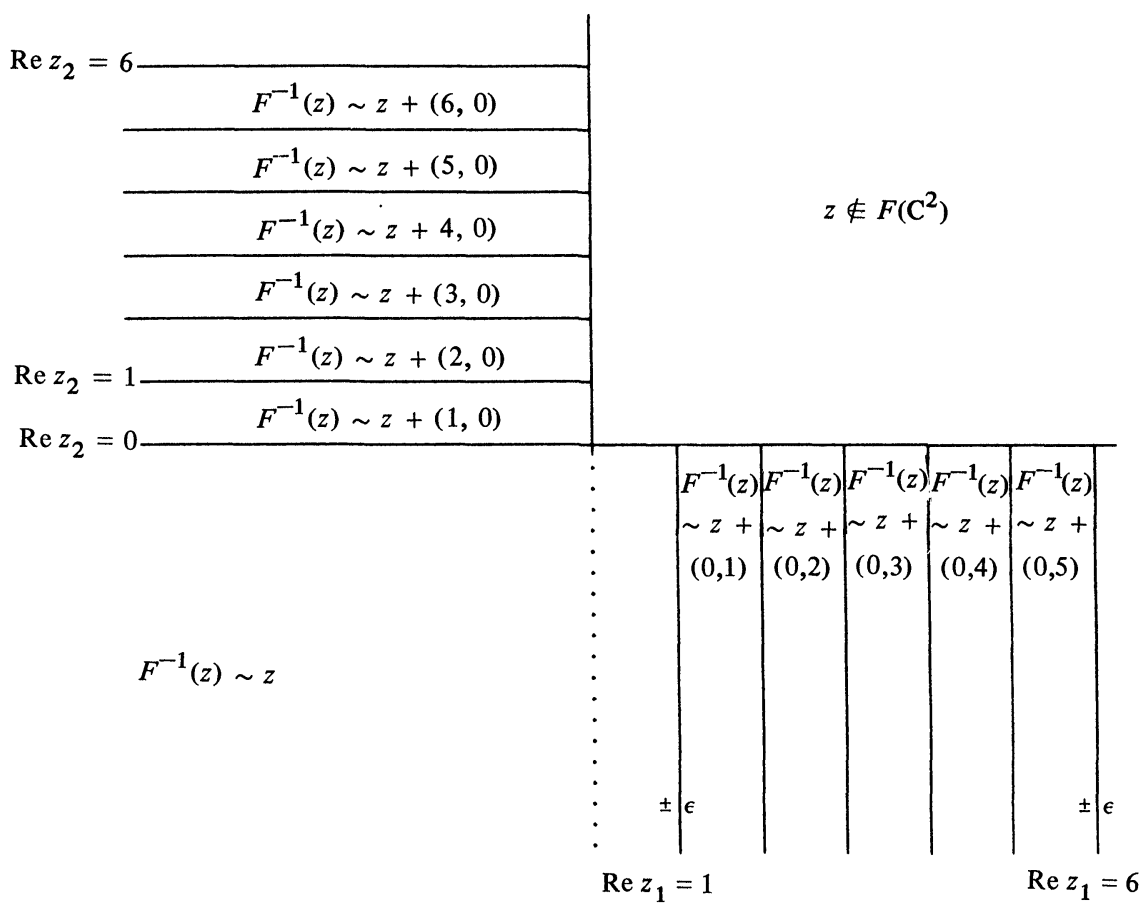

FIGURE 1. The behavior of $F^{-1}$ and the critical tube $V_{\varepsilon}=\left\{z \in \mathbf{C}^{2} \mid d(z, A)<\varepsilon\right\}$ (Theorem 8.9(1)-(2)).

Note that in the above theorem we did not use the full force of Lemma 8.7. Since in Lemma 8.7 we have $|\Theta(z)-z|<\eta e^{-\rho(z)^{1 / 4}}$ for $\operatorname{Re} \pi_{1}(z) \leqslant a-\delta$, $\operatorname{Re} \pi_{2}(z) \leqslant b-\mu-\delta$, we see that $\Theta(z)-z$ converges to zero when $\rho(z)$ goes to infinity for $\operatorname{Re} \pi_{1}(z) \leqslant a-\delta, \operatorname{Re} \pi_{2}(z) \leqslant b-\mu-\delta$. A similar remark holds for the other conditions of the lemma. So, the larger $\min \left(\left|\operatorname{Im} \pi_{1}(z)\right|\right.$, $\left.\left|\operatorname{Im} \pi_{2}(z)\right|\right)$ is, the better is the information which can be obtained about the difference $F(z)-S(z)$ outside the critical tube $W_{\varepsilon}$. By using Lemma 8.8 we can then replace the critical tube $V_{\varepsilon}=\left\{z \in \mathbf{C}^{2} \mid d(z, A) \geqslant \varepsilon\right\}$ by a smaller set and improve the information about the difference $F^{-1}(z)-T(z)$ on the intersection of $\Omega$ with the complement of this smaller set.

Note also that the weight $e^{-|z|^{1 / 4}}$ is not the best possible in Lemma 8.1. Weights $\omega$ which satisfy

$$
\int_{1}^{\infty} \frac{|\log \omega(t)|}{t^{3 / 2}} d t<+\infty
$$

work for all closed sets which satisfy Arakelian's condition, but for a union of vertical strips better weights can be found [7]. Also, in Lemma 8.1, we can replace the vertical exceptional strips by subsets of $\mathbf{C}$ which get infinitely thin when $|\operatorname{Im} \xi| \rightarrow \infty$. This could also lead to versions of Theorem 8.9 where the critical tubes $V_{\varepsilon}$ and $W_{\varepsilon}$ are replaced by smaller sets, and where the information about the differences $F^{-1}(z)-T(z)$ and $F(z)-S(z)$ outside these critical sets is improved. 


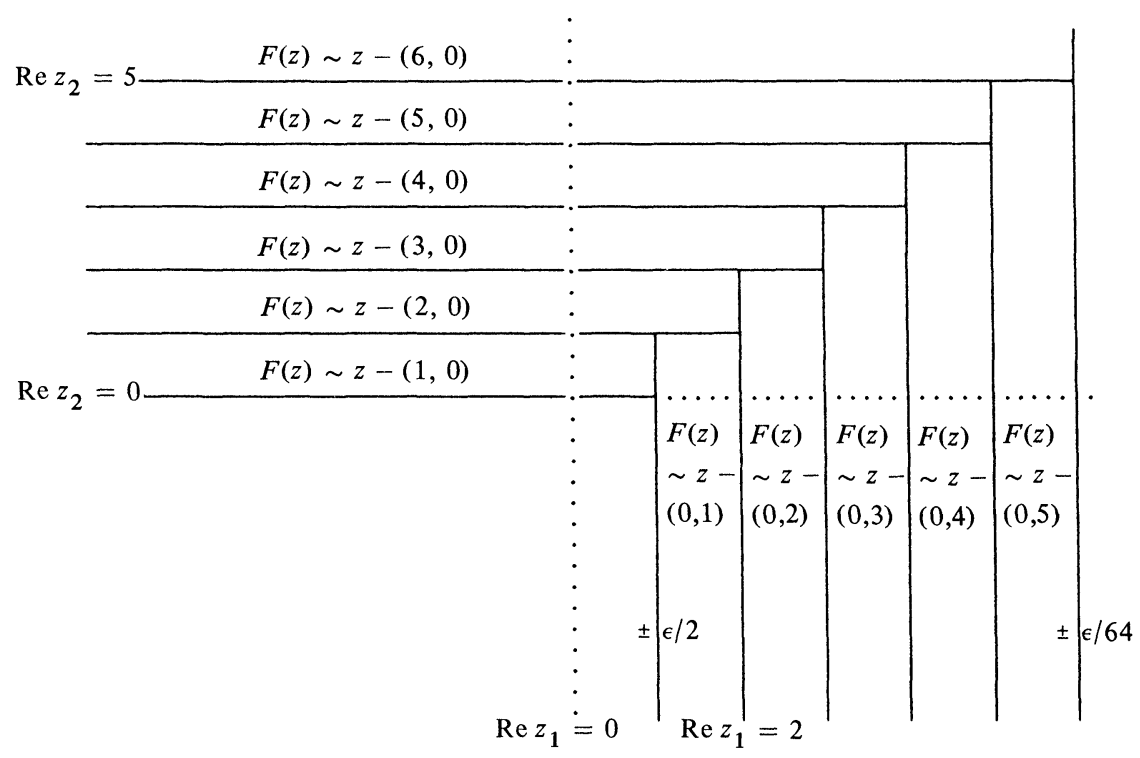

Figure 2. The behavior of $F$ and the critical tube $W_{\varepsilon}$ (Theorem $\left.8.9(3)\right)$.

Statements as precise as Theorem 8.9 can be obtained by playing a more complicated "Arakelian game". Each automorphism $\Theta_{n}$ in the construction of $F$ consists in giving one vertical translation on a vertical tube, when "freezing" the complement (except for a critical vertical tube) and then in giving one horizontal translation on a horizontal tube, when "freezing" the complement (except for a critical horizontal tube). But we could introduce at each step various vertical translations on various vertical tubes, and then various horizontal translations on various horizontal tubes. We can even have at each step infinitely many vertical and horizontal translations.

The sequence $\left(\Theta_{1} \circ \ldots \circ \Theta_{n}\right)$ will converge to an entire mapping $F$ if $\sum_{n=1}^{\infty}\left\|I-\Theta_{n}\right\|_{K}<+\infty$ for each compact set $K$, and we can arrange this condition to be satisfied not only for compact sets but also for tubes with compact basis. Of course, the critical sets which will appear in such more sophisticated constructions will be more complicated than the critical tubes $V_{\varepsilon}$ and $W_{\varepsilon}$. We leave the details to the reader, and we will not study here the kind of ranges which can be obtained for such Bieberbach functions $F$.

Condition (7) in Theorem 8.9 ensures that if $\Delta$ is a bounded subset of $\mathbf{C}^{2}$ then the set $\left\{z \in F^{-1}(\Delta) \mid \sup \left(\operatorname{Re} \pi_{1}(z), \operatorname{Re} \pi_{2}(z)\right) \leqslant a\right\}$ is bounded for every $a \in \mathbf{R}$. In fact we showed that $|z| \leqslant \sup _{u \in \Delta}\left|F_{m}^{-1}(u)\right|+\varepsilon / 2^{m+1}$ if $z \in F^{-1}(\Delta)$ and if $\max \left(\operatorname{Re} \pi_{1}(z), \operatorname{Re} \pi_{2}(z)+1\right) \leqslant m$. In order for $F$ to satisfy the conditions of Theorem 8.9 we can use a sequence $\left(\Theta_{m}\right)$ of elements of Aut $_{1}\left(\mathbf{C}^{2}\right)$ where, for each $m \geqslant 1, \Theta_{m}$ is the map $(x, y) \rightarrow(u, v)$, with $v=$ $y-h_{m}(x-m), u=x-h_{m}(v-m+1)$, and where $h_{m}$ is an entire function 


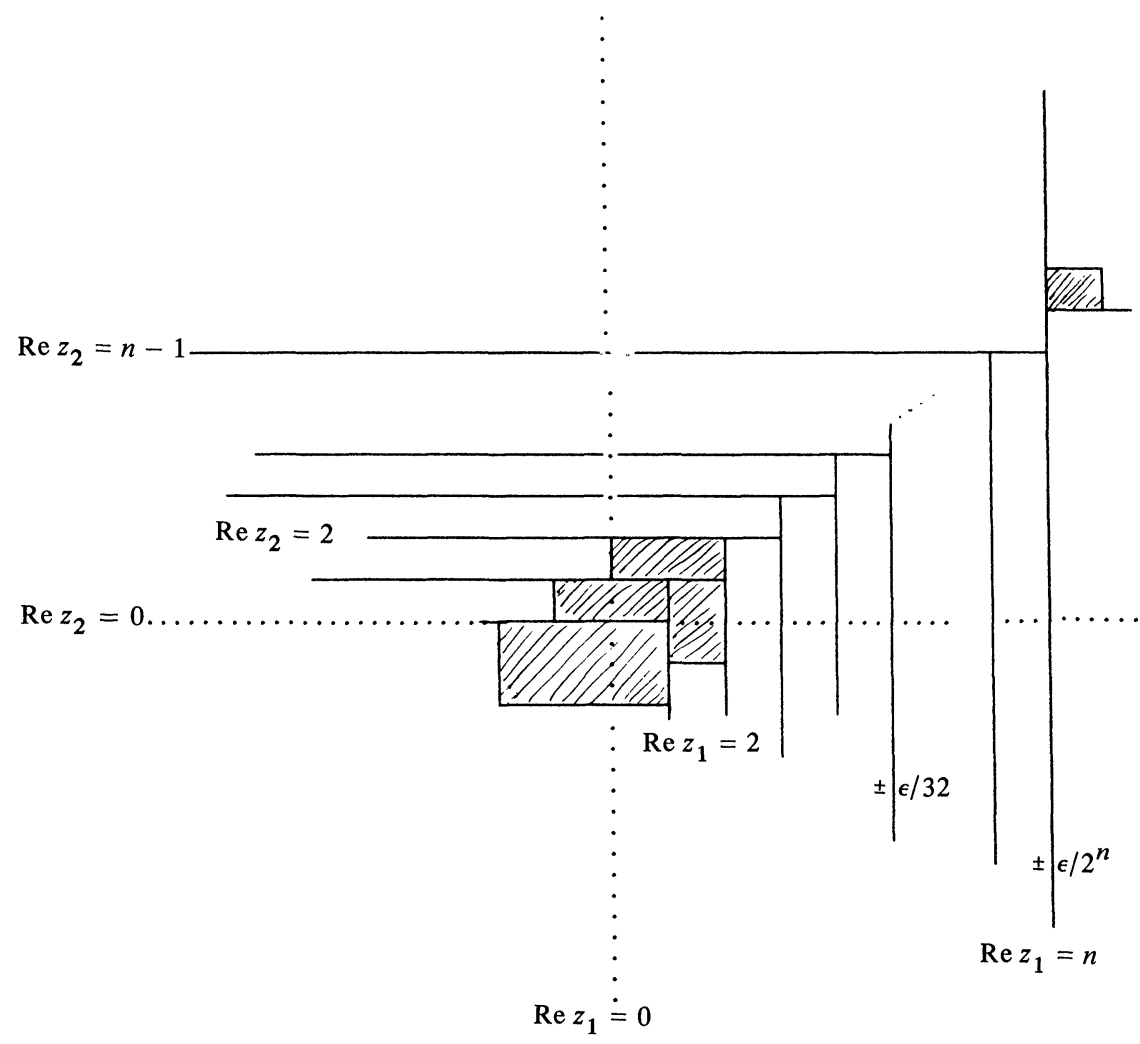

Figure 3. $F_{n}^{-1}(D(0,2))$ (condition (10), proof of Theorem 8.9).

over $\mathbf{C}$ which satisfies

$$
\begin{aligned}
&\left|h_{m}(\xi)\right|<\varepsilon / 2^{m+1}\left(\operatorname{Re} \xi \leqslant-\varepsilon / 2^{m+2}\right), \\
&\left|h_{m}(\xi)-1\right|<\varepsilon / 2^{m+1} \quad\left(\operatorname{Re} \xi \geqslant \varepsilon / 2^{m+2}\right) .
\end{aligned}
$$

This sequence $\left(h_{m}\right)$ of entire functions can be obtained by using Arakelian's theorem only once. To see this consider an entire function $h$ which is such that $|h(\xi)|<e^{-|\xi|^{1 / 4}}(\operatorname{Re} \xi \leqslant-1 / 2),|h(\xi)-1|<e^{-|\xi|^{1 / 4}}(\operatorname{Re} \xi \geqslant 1 / 2)$. Set

$$
h_{m}(\xi)=h\left[\frac{2^{m+2}}{\varepsilon}\left(\log \left(2^{m+1} \varepsilon^{-1}\right)\right)^{4} \xi\right] \quad(\xi \in \mathbf{C}, m \geqslant 1) .
$$

This sequence $\left(h_{m}\right)_{m \geqslant 1}$ satisfies the required conditions, and we can deduce estimates for $\left\|F_{m}^{-1}\right\|_{\Delta}=\sup _{u \in \Delta}\left|F_{m}^{-1}(u)\right|$ from estimates for the growth of the function $h$. We will not do this here.

We showed at the end of $\S 7$ that if $F$ is Bieberbach's original example there exists $K>0$ such that $(F \circ G)\left(\mathbf{C}^{2}\right) \cap D(0, K) \neq \varnothing$ for every nonconstant entire mapping $G: \mathbf{C}^{2} \rightarrow \mathbf{C}^{2}$. We do not know whether a similar property holds for the mappings constructed in Theorem 8.9. 


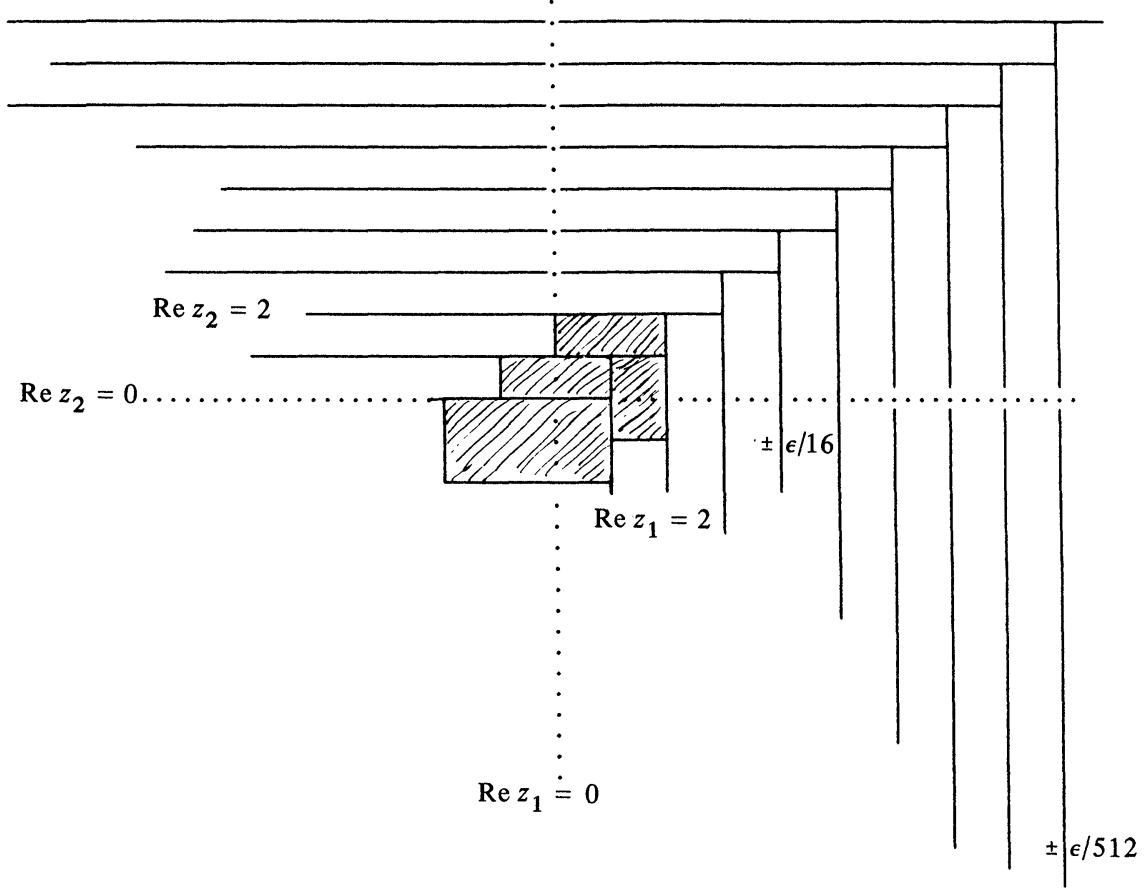

Figure 4. $F^{-1}(D(0,2))$ (Theorem $8.9(6)$ and Theorem 8.11).

Nevertheless it is possible, for these functions $F$, to obtain more qualitative information about inverse images of open sets which meet the boundary of $F\left(\mathbf{C}^{2}\right)$.

We will need the following well-known result.

LEMMA 8.10. Let $U$ be a connected open subset of $\mathbf{C}$ and let $\left(f_{n}\right)$ be a sequence of holomorphic functions over $U$. If there exists $\alpha, \beta \in U$ such that

$$
\lim \sup _{n \rightarrow \infty}\left|f_{n}(\alpha)\right|<+\infty, \quad \liminf _{n \rightarrow \infty}\left|f_{n}(\beta)\right|=+\infty,
$$
then $\mathbf{C} \backslash \bigcup_{n \geqslant 1} f_{n}(U)$ contains at most one point.

Proof. It follows from [27, Chapter 12, Theorem 4.1] that if $\mathbf{C} \backslash$ $\left(\cup_{n \geqslant 1} f_{n}(U)\right)$ had more than one element then either the sequence $\left(f_{n}\right)$ would have a normal subsequence or the sequence $\left(f_{n}\right)$ would have a subsequence $\left(f_{n_{t}}\right)$ such that $\left|f_{n_{i}}(z)\right|_{i \rightarrow \infty}^{\rightarrow} \infty$ uniformly over compact subsets of $U$. Since the hypotheses exclude both possibilities, the result follows.

In the following theorem, we use the same notations as in the proof of Theorem 8.9.

THEOREM 8.11. Let $F: \mathbf{C}^{2} \rightarrow \mathbf{C}^{2}$ be an entire mapping. For $i=1,2, \Delta \subset \mathbf{C}^{2}$ set $\lambda_{i, n}(\Delta)=\inf \left\{\lambda=\operatorname{Re} \pi_{i}(u) \mid u \in F^{-1}(\Delta), \quad \operatorname{Re} \pi_{1}(u) \leqslant n, \quad \operatorname{Re} \pi_{2}(u) \leqslant n\right\}$. Assume that $F_{n} \underset{n \rightarrow \infty}{\stackrel{\sigma}{\rightarrow}} F$ where $\left(F_{n}\right)$ is a sequence of elements of $\operatorname{Aut}\left(\mathbf{C}^{2}\right)$ which possesses the following properties.

(i) $\left|F_{n}(z)-S_{n}(z)\right| \leqslant 1 / 2\left(d\left(z, B_{n}\right)>1 / 2, n \geqslant 1\right)$. 
(ii) There exists a sequence $\left(\varepsilon_{n}\right)$ of positive real numbers which converges to zero such that

$\left|\left(F_{m}^{-1} \circ F_{n}\right)(z)-z\right| \leqslant \varepsilon_{n}$

$$
\left(\operatorname{Re} \pi_{1}(z) \leqslant n+1 / 2, \operatorname{Re} \pi_{2}(z) \leqslant n-1 / 2, m \geqslant n \geqslant 1\right) .
$$

Then $\pi_{1}\left(F^{-1}(U)\right)$ and $\pi_{2}\left(F^{-1}(U)\right)$ are dense in $\mathbf{C}$ and

$$
\limsup _{n \rightarrow \infty} \frac{\lambda_{1, n}(U)}{n}=\limsup _{n \rightarrow \infty} \frac{\lambda_{2, n}(U)}{n}=-\infty
$$

for every open subset $U$ of $\mathbf{C}^{2}$ which is such that $U \cap \operatorname{Fr}\left(F\left(\mathbf{C}^{2}\right)\right) \neq \varnothing$.

Proof. Let $z \in \mathbf{C}^{2}$ and let $n>1 / 2+\sup \left(\operatorname{Re} \pi_{1}(z), \operatorname{Re} \pi_{2}(z)+1\right)$. Set $u=F_{n}^{-1}(z)$, and assume that $\inf \left(\operatorname{Re} \pi_{1}(u), \operatorname{Re} \pi_{2}(u)\right)<n-1 / 2$. If $d\left(u, B_{n}\right) \leqslant$ $1 / 2$, then it follows from the geometry of $B_{n}$ that $\sup \left(\operatorname{Re} \pi_{1}(u), \operatorname{Re} \pi_{2}(u)+1\right)$ $\leqslant n+1 / 2$.

If $d\left(u, B_{n}\right)>1 / 2$, then it follows from (i) that $\left|z-S_{n}(u)\right| \leqslant 1 / 2$, so that $\operatorname{Re}\left(\pi_{1} \circ S_{n}\right)(u)<n, \operatorname{Re}\left(\pi_{2} \circ S_{n}\right)(u)<n-1$. If

$$
\inf \left(\operatorname{Re}\left(\pi_{1} \circ S_{n}\right)(u), \operatorname{Re}\left(\pi_{2} \circ S_{n}\right)(u)\right)>0,
$$

then, since $T_{n}=S_{n}^{-1}$, it would follow from the definition of $T_{n}$ that $\inf \left(\operatorname{Re} \pi_{1}(u), \operatorname{Re} \pi_{2}(u)\right)>n$, a contradiction. So either $\operatorname{Re}\left(\pi_{1} \circ S_{n}\right)(u)<0$, $\operatorname{Re}\left(\pi_{2} \circ S_{n}\right)(u)<n-1$, or $\operatorname{Re}\left(\pi_{1} \circ S_{n}\right)(u)<n, \operatorname{Re}\left(\pi_{2} \circ S_{n}\right)(u)<0$. By using the definition of $T_{n}$ and the fact that $T_{n}=S_{n}^{-1}$, we obtain in the first case $\operatorname{Re} \pi_{1}(u)<n-1, \operatorname{Re} \pi_{2}(u)<n-1$, and in the second case $\operatorname{Re} \pi_{1}(u)<n$, $\operatorname{Re} \pi_{2}(u)<n-1$. Thus, we have the property

(a) If $\inf \left(\operatorname{Re}\left(\pi_{1} \circ F_{n}^{-1}\right)(z), \operatorname{Re}\left(\pi_{2} \circ F_{n}^{-1}\right)(z)\right)<n-1 / 2$, and if $n>1 / 2+$ $\sup \left(\operatorname{Re} \pi_{1}(z), \operatorname{Re} \pi_{2}(z)+1\right)$, then

$$
\sup \left(\operatorname{Re}\left(\pi_{1} \circ F_{n}^{-1}\right)(z), \operatorname{Re}\left(\pi_{2} \circ F_{n}^{-1}\right)(z)+1\right) \leqslant n+1 / 2 \quad\left(z \in \mathbf{C}^{2}\right) .
$$

Also, we deduce immediately from (ii) the following property.

(b) If $\sup \left(\operatorname{Re}\left(\pi_{1} \circ F_{n}^{-1}\right)(z), \operatorname{Re}\left(\pi_{2} \circ F_{n}^{-1}\right)(z)+1\right) \leqslant n+1 / 2$, then $z \in$ $F\left(\mathbf{C}^{2}\right)$, and $\left|F^{-1}(z)-F_{n}^{-1}(z)\right| \leqslant \varepsilon_{n}\left(z \in \mathbf{C}^{2}, n \geqslant 1\right)$.

By using (a) and (b), we obtain

$$
\begin{gathered}
\inf \left(\operatorname{Re}\left(\pi_{1} \circ F_{n}^{-1}\right)(z), \operatorname{Re}\left(\pi_{2} \circ F_{n}^{-1}\right)(z)\right) \geqslant n-1 / 2 \\
\left(z \in \mathbf{C}^{2} \backslash F\left(\mathbf{C}^{2}\right), \quad n>1 / 2+\sup \left(\operatorname{Re} \pi_{1}(z), \operatorname{Re} \pi_{2}(z)+1\right) .\right.
\end{gathered}
$$

Now let $U$ be an open subset of $\mathbf{C}^{2}$ such that $U \cap \operatorname{Fr}\left(F\left(\mathbf{C}^{2}\right)\right) \neq \varnothing$, and let $z_{0} \in U \cap \operatorname{Fr}\left(F\left(\mathbf{C}^{2}\right)\right.$. There exists $z_{1} \in F\left(\mathbf{C}^{2}\right)$ such that $D\left(z_{0}, 2\left|z_{1}-z_{0}\right|\right) \subset U$.

Denote by $D$ the open unit disc of the complex plane. For $\xi \in D$ set $\omega(\xi)=2 \xi z_{1}+(1-2 \xi) z_{0}$.

Fix $i \in\{1,2\}$ and set $h_{n}=\pi_{i} \circ F_{n}^{-1} \circ \omega(n \geqslant 1)$. Then $\operatorname{Re} h_{n}(0)=$ $\operatorname{Re}\left(\pi_{i} \circ F_{n}^{-1}\right)\left(z_{0}\right)$ and so it follows from (c) that $\liminf _{n \rightarrow \infty}\left|h_{n}(0)\right|=+\infty$. Also $h_{n}(1 / 2)=\left(\pi_{i} \circ F_{n}^{-1}\right)\left(z_{1}\right)$ and so it follows from Corollary 5.3 (3) that $\left(\pi_{i} \circ F^{-1}\right)\left(z_{1}\right)=\lim _{n \rightarrow \infty} h_{n}(1 / 2)$.

Fix $\beta \in \mathbf{C}, \eta>0$. Set $n_{0}=\sup \left\{n \in \mathbf{N} \mid \varepsilon_{n} \geqslant \eta / 2\right\}$. Let $n_{1}>\sup \left(n_{0}, \operatorname{Re} \beta\right.$ $\left.+1 / 2+\eta / 2,\left|z_{0}\right|+3 / 2+2\left|z_{1}-z_{0}\right|\right)$. It follows from Lemma 8.10 that $\cup_{n \geqslant n_{1}} h_{n}(D)$ is dense in $\mathbf{C}$, and so there exists $u \in D\left(z_{0}, 2\left|z_{1}-z_{0}\right|\right)$ which satisfies $\left|\beta-\left(\pi_{i} \circ F_{n}^{-1}\right)(u)\right|<\eta / 2$ for some $n \geqslant n_{1}$. So $n>\sup \left(\operatorname{Re} \pi_{i}(u)\right.$, 
$\left.\operatorname{Re} \pi_{2}(u)+1\right)+1 / 2$, and $\operatorname{Re}\left(\pi_{i} \circ F_{n}^{-1}\right)(u)<\operatorname{Re} \beta+\eta / 2<n-1 / 2$. It follows then from (a) and (b) that $u \in F\left(\mathbf{C}^{2}\right)$, and that $\left|F_{n}^{-1}(u)-F^{-1}(u)\right| \leqslant \varepsilon_{n}$ $<\eta / 2$. Hence $\left|\beta-\left(\pi_{i}^{\circ} \circ F^{-1}\right)(u)\right|<\eta$, and $\pi_{i}\left(F^{-1}(U)\right)$ is dense in $\mathbf{C}$. This proves the first assertion.

Also there exists $r>0$ which is such that the closed ball $\overline{B\left(z_{0}, r\right)}$ is contained in $U$, and there exists a sequence $\left(u_{p}\right)$ of elements of the open ball $B\left(z_{0}, r / 2\right)$ which is such that $u_{p} \in F\left(\mathbf{C}^{2}\right)(p \geqslant 1)$, and such that $z_{0}=$ $\lim _{p \rightarrow \infty} u_{p}$.

Denote by $B_{p}$ the closed ball $\overline{B\left(u_{p}, r-\left|u_{p}-z_{0}\right|\right)}$, and denote by $B_{p}^{\prime}$ the closed ball $\overline{B\left(z_{0}, r-2\left|u_{p}-z_{0}\right|\right)}(p \geqslant 1)$. Let $m$ be the Lebesgue measure on $C^{p}$. We have $B_{p}^{\prime} \subset B_{p} \subset \stackrel{U}{U}(p \geqslant 1)$ and

$$
\lim _{p \rightarrow \infty} m\left(B_{p}^{\prime}\right)=\lim _{p \rightarrow \infty} m\left(B_{p}\right)=m\left(\overline{B\left(z_{0}, r\right)}\right) .
$$

Fix again $i \in\{1,2\}$. It follows from the harmonicity of the function $z \rightarrow \operatorname{Re}\left(\pi_{i} \circ F_{n}^{-1}\right)(z)$ that

$$
m\left(B_{p}\right) \operatorname{Re}\left(\pi_{i} \circ F_{n}^{-1}\right)\left(u_{p}\right)=\int_{B_{p}} \operatorname{Re}\left(\pi_{i} \circ F_{n}^{-1}\right)(\xi) d \xi
$$

and that

$$
m\left(B_{p}^{\prime}\right) \operatorname{Re}\left(\pi_{i} \circ F_{n}^{-1}\right)\left(z_{0}\right)=\int_{B_{p}^{\prime}} \operatorname{Re}\left(\pi_{i} \circ F_{n}^{-1}\right)(\xi) d \xi .
$$

Set $\mu_{n}=\inf \left\{\mu=\operatorname{Re}\left(\pi_{i} \circ F_{n}^{-1}\right)(u) \mid u \in U\right\}$. By using (c), we obtain

$$
\begin{aligned}
\mu_{n} m\left(B_{p} \backslash B_{p}^{\prime}\right) & \leqslant \int_{B_{p} \backslash B_{p}^{\prime}} \operatorname{Re}\left(\pi_{i} \circ F_{n}^{-1}\right)(\xi) d \xi \\
& =m\left(B_{p}\right) \operatorname{Re}\left(\pi_{i} \circ F_{n}^{-1}\right)\left(u_{p}\right)-m\left(B_{p}^{\prime}\right) \operatorname{Re}\left(\pi_{i} \circ F_{n}^{-1}\right)\left(z_{0}\right) \\
& \leqslant m\left(B_{p}\right) \operatorname{Re}\left(\pi_{i} \circ F_{n}^{-1}\right)\left(u_{p}\right)-m\left(B_{p}^{\prime}\right)(n-1 / 2) \\
(p \geqslant 1, & \left.n>\sup \left(\operatorname{Re} \pi_{1}\left(z_{0}\right)+1 / 2, \operatorname{Re} \pi_{2}\left(z_{0}\right)+3 / 2\right)\right)
\end{aligned}
$$

Since $\left(\pi_{i} \circ F^{-1}\right)\left(u_{p}\right)=\lim _{n \rightarrow \infty}\left(\pi_{i} \circ F_{n}^{-1}\right)\left(u_{p}\right)$, we have

$$
\limsup _{n \rightarrow \infty} \frac{\mu_{n}}{n} \leqslant \frac{-m\left(B_{p}^{\prime}\right)}{m\left(B_{p} \backslash B_{p}^{\prime}\right)}
$$

for every $p \geqslant 1$, hence $\lim \sup _{n} / n=-\infty$. So there exists a sequence $\left(v_{n}\right)$ of elements of $U$ such that

$$
\limsup _{n \rightarrow \infty} \frac{\operatorname{Re}\left(\pi_{i} \circ F_{n}^{-1}\right)\left(v_{n}\right)}{n}=-\infty .
$$

Since $U$ is bounded, it follows from (a) and (b) that

$$
\sup \left(\operatorname{Re}\left(\pi_{1} \circ F_{n}^{-1}\right)\left(v_{n}\right), \operatorname{Re}\left(\pi_{2} \circ F_{n}^{-1}\right)\left(v_{n}\right)+1\right) \leqslant n+1 / 2,
$$


that $v_{n} \in F\left(\mathbf{C}^{2}\right)$ and that $\left|F^{-1}\left(v_{n}\right)-F_{n}^{-1}\left(v_{n}\right)\right| \leqslant \varepsilon_{n}$ when $n$ is sufficiently large. We obtain

$$
\limsup _{n \rightarrow \infty} \frac{\lambda_{i, n}}{n} \leqslant \limsup _{n \rightarrow \infty} \frac{\operatorname{Re}\left(\pi_{i} \circ F^{-1}\right)\left(v_{n-1}\right)}{n}=-\infty .
$$

This concludes the proof of the theorem.

The mappings $F$ constructed as in the proof of Theorem 8.9 satisfy the hypothesis of Theorem 8.11, since condition (i) is weaker than (8) and condition (ii) weaker than (12). We do not know whether Theorem 8.11 applies to all mappings which satisfy the conditions of Theorem 8.9, since some specific properties of the sequence $\left(F_{n}\right)$ used to construct $F$ were used in the proof of Theorem 8.11. But the other tools of the proof of Theorem 8.11, the mean value formula and Lemma 8.10, can, of course, be used to study $F^{-1}(U)$ if $F$ is any Bieberbach mapping and if $U$ is any neighborhood of an element of the boundary of $F\left(C^{2}\right)$. This suggests that results similar to Theorem 8.11 might hold in this general setting. Note that the classical theorem [27, Chapter 12, Theorem 4.1] used to prove Lemma 8.10 is a deep result, since the big Picard theorem follows immediately from it. But in the proof of Theorem 8.11 we just used the fact that $U_{n \geqslant n_{1}} h_{n}(D)$ is dense in $\mathbf{C}$. This can be shown directly, since otherwise there would exist $a \in \mathbf{C}$ such that the sequence $\left(1 /\left(h_{n}-a\right)\right)_{n \geqslant n_{1}}$ is bounded over $D$, and the limit of any subsequence of it would either never vanish or vanish everywhere on $D$, by Rouché's theorem. This argument, similar to the standard proof of Weierstrass's density theorem, shows how elementary are the reasons which force $\pi_{1}\left(F^{-1}(U)\right)$ and $\pi_{2}\left(F^{-1}(U)\right)$ to be dense in $\mathbf{C}$ if $U$ is an open neighborhood of an element of the boundary of $F\left(\mathbf{C}^{2}\right)$, when $F$ is constructed as in the proof of Theorem 8.9.

Also, the second assertion of Theorem 8.11 shows that the branches of $F^{-1}(U) \cap W_{\varepsilon}$ which appear in Figure 4 get infinitely long at infinity. We now use this fact to obtain the following result, which shows how complicated the range of a Bieberbach mapping can be.

COROLlary 8.12. There exists a Bieberbach mapping $G: \mathbf{C}^{2} \rightarrow \mathbf{C}^{2}$ such that

(i) $\operatorname{Fr}\left(G\left(\mathbf{C}^{2}\right)\right) \subset \operatorname{Fr}(G \circ G)\left(\mathbf{C}^{2}\right)$,

(ii) $(G \circ G)\left(\mathbf{C}^{2}\right) \cap U$ has infinitely many components for every bounded subset $U$ of $\mathbf{C}^{2}$ whose intersection with the boundary of $G\left(\mathbf{C}^{2}\right)$ is nonempty.

Proof. Apply the construction of Theorem 8.9 with $\varepsilon=1 / 4$. Let $\tau$ be the translation $z \rightarrow z-(1 / 2,1 / 2)$. Let $G=F \circ \tau$. By Theorem 8.9 (1), (2) we have $\Omega \backslash A_{\varepsilon} \subset F\left(\mathbf{C}^{2}\right) \subset\left\{z: \inf \left(\operatorname{Re} \pi_{1}(z), \operatorname{Re} \pi_{2}(z)\right)<1 / 4\right\}$. Hence

$$
\begin{aligned}
& W_{\varepsilon} \cap\left\{z: \inf \left(\operatorname{Re} \pi_{1}(z), \operatorname{Re} \pi_{2}(z)\right)\right.\leqslant-3 / 4\} \\
& \subset(\tau \circ F)\left(\mathbf{C}^{2}\right) \subset\left\{z: \inf \left(\operatorname{Re} \pi_{1}(z), \operatorname{Re} \pi_{2}(z)\right)<-1 / 4\right\} .
\end{aligned}
$$

It follows that $W_{\varepsilon} \cap(\tau \circ F)\left(\mathbf{C}^{2}\right)$ has infinitely many components.

Now let $U$ be a bounded open subset of $\mathbf{C}^{2}$ whose intersection with the boundary of $G\left(\mathbf{C}^{2}\right)=F\left(\mathbf{C}^{2}\right)$ is nonempty. By Theorem 8.9(6), $F^{-1}(U) \subset W_{\varepsilon}$ $\cup T\left(U_{\varepsilon} \cap \Omega\right)$. By using Theorem 8.11 and Theorem 8.9(7) we find that 
$F^{-1}(U) \cap W_{\varepsilon} \cap(\tau \circ F)\left(C^{2}\right)$ has infinitely many components which do not meet the bounded open set $T\left(U_{\varepsilon} \cap \Omega\right)$.

Since $\left(G^{2} \circ \tau^{-1}\right)\left(\mathbf{C}^{2}\right) \cap U=F\left(F^{-1}(U) \cap(\tau \circ F)\left(\mathbf{C}^{2}\right)\right)$, we see that $G^{2}\left(\mathbf{C}^{2}\right)$ $\cap U=\left(G^{2} \circ \tau^{-1}\right)\left(\mathbf{C}^{2}\right) \cap U$ has infinitely many components. This proves (ii). But it follows from (ii) that $\operatorname{Fr}\left(G\left(\mathbf{C}^{2}\right)\right) \subset \overline{G^{2}\left(\mathbf{C}^{2}\right)}$, and so $\operatorname{Fr}\left(G\left(\mathbf{C}^{2}\right)\right) \subset$ $\operatorname{Fr}\left(G^{2}\left(\mathbf{C}^{2}\right)\right)$, since $\operatorname{Fr}\left(G\left(\mathbf{C}^{2}\right)\right) \cap G\left(\mathbf{C}^{2}\right)=\varnothing$. This proves the corollary.

We showed at the beginning of this section that the range of an entire nondegenerate function on $\mathbf{C}^{2}$ can be very small. We now deduce from Theorem 8.9 examples which show that the range of an entire nondegenerate function on $\mathbf{C}^{2}$ can be both nondense and very large (of course, as we observed in $\S 4$, the entire functions obtained below cannot be one-to-one).

THEOREM 8.13. Let $\varepsilon>0$, and let $n \geqslant 3$ be an integer satisfying $(1+\varepsilon)$ $\cdot(\cos (\pi / n))^{n}>1$. Then there exists an entire function $G: \mathbf{C}^{2} \rightarrow \mathbf{C}^{2}$ which possesses the following properties.

(1) $G\left(\mathbf{C}^{2}\right) \cap \overline{D(0,1)}=\varnothing$.

(2) $\mathbf{C}^{2} \backslash D(0,1+\varepsilon) \subset G\left(\mathbf{C}^{2}\right)$.

(3) For every $z \in G\left(\mathbf{C}^{2}\right)$, the set $G^{-1}(\{z\})$ possesses at most $n^{2}$ elements.

Proof. Set $\alpha=(1+\varepsilon)^{1 / n} \cos (\pi / n)-1$, so that $0<\alpha<\varepsilon$, and let $m$ be an integer such that $m \geqslant \alpha+\varepsilon+2$. Let $F$ be a Bieberbach function satisfying the conditions of Theorem 8.9 with $\varepsilon=\alpha / 2 m$. So $z \in F\left(\mathbf{C}^{2}\right)$ if $\operatorname{Re} \pi_{1}(z) \leqslant 1$ $-\alpha / 2 m, \operatorname{Re} \pi_{2}(z) \leqslant-\alpha / 2 m$, or if $\alpha / 2 m \leqslant \operatorname{Re} \pi_{2}(z) \leqslant 1-\alpha / 2 m, \operatorname{Re} \pi_{1}(z)$ $\leqslant-\alpha / 2 m$, and $z \notin F\left(\mathbf{C}^{2}\right)$ if $\operatorname{Re} \pi_{1}(z) \geqslant \alpha / 2 m, \operatorname{Re} \pi_{2}(z) \geqslant \alpha / 2 m$. There exists an affine map $L$ such that the function $H=L \circ F$ satisfies the conditions

(i) If $\operatorname{Re} \pi_{2}(z) \geqslant 1+\alpha, \operatorname{Re} \pi_{1}(z) \geqslant-m+1+\alpha$, then $z \in H\left(\mathbf{C}^{2}\right)$.

(ii) If $-m+1+\alpha \leqslant \operatorname{Re} \pi_{2}(z) \leqslant 1, \operatorname{Re} \pi_{1}(z) \geqslant 1+\alpha$, then $z \in H\left(\mathbf{C}^{2}\right)$.

(iii) If $\operatorname{Re} \pi_{1}(z) \leqslant 1, \operatorname{Re} \pi_{2}(z) \leqslant 1$ then $z \notin H\left(\mathbf{C}^{2}\right)$.

Consider the function $\psi: z \rightarrow\left(\left(\pi_{1}(z)\right)^{n},\left(\pi_{2}(z)\right)^{n}\right)$, and set $G=\psi \circ H$. Clearly, $G$ satisfies (3). If $z \in \mathbf{C}^{2}$, then

$$
\sup \left(\operatorname{Re}\left(\pi_{1} \circ H\right)(z), \operatorname{Re}\left(\pi_{2} \circ H\right)(z)\right)>1,
$$

so that

$$
\begin{aligned}
& \sup \left(\left|\left(\pi_{1} \circ H\right)(z)\right|,\left|\left(\pi_{2} \circ H\right)(z)\right|\right)>1, \\
& \sup \left(\left|\left(\pi_{1} \circ G\right)(z)\right|,\left|\left(\pi_{2} \circ G\right)(z)\right|\right)>1
\end{aligned}
$$

and hence $G$ satisfies (1).

Now let $u=\left(\rho_{1} e^{i \Theta_{1}}, \rho_{2} e^{i \Theta_{2}}\right)$ with $\left|\Theta_{1}\right| \leqslant \pi,\left|\Theta_{2}\right| \leqslant \pi, \rho_{1} \geqslant 0, \rho_{2} \geqslant 0$, be an element of $\mathbf{C}^{2} \backslash D(0,1+\varepsilon)$. If $\rho_{2} \geqslant 1+\varepsilon$ set $v_{2}=\rho_{2}^{1 / n} \cdot e^{i \Theta_{2} / n}$. Then $\operatorname{Re} v_{2} \geqslant$ $(1+\varepsilon)^{1 / n} \cos (\pi / n)=1+\alpha$. Let $\xi$ be an $n$th root of $e^{i \Theta_{1}}$ with $\operatorname{Re} \xi \geqslant 0$, and set $v_{1}=\rho_{1}^{1 / n} \xi$. Then $v=\left(v_{1}, v_{2}\right) \in H\left(\mathbf{C}^{2}\right)$, so that $u=\psi(v) \in G\left(\mathbf{C}^{2}\right)$.

If $\rho_{2}<1+\varepsilon$, then $\rho_{1} \geqslant 1+\varepsilon$. Set $v_{1}=\rho_{1}^{1 / n} e^{i \Theta_{1} / n}$. We see as above that $\operatorname{Re} v_{1} \geqslant 1+\alpha$. Let $\xi$ be an $n$th root of $e^{i \Theta_{2}}$ with $\operatorname{Re} \xi \leqslant 0$, and set $v_{2}=\rho_{2}^{1 / n} \xi$. Then $0 \geqslant \operatorname{Re} v_{2} \geqslant-\rho_{2}^{1 / n}>-1-\varepsilon \geqslant \alpha-m+1$. So $v=\left(v_{1}, v_{2}\right) \in H\left(\mathbf{C}^{2}\right)$, and $u=\psi(v) \in G\left(\mathbf{C}^{2}\right)$. Hence $\mathbf{C}^{2} \backslash D(0,1+\varepsilon) \subset G\left(\mathbf{C}^{2}\right)$, and the theorem is proved. 
We can obtain, with $n=3, \varepsilon=8$, an entire function whose range avoids $\overline{D(0,1)}$ and contains the complement of $D(0,9)$ such that the inverse image of ( $z$ ) never contains more than 9 elements. If we take $n=4, \varepsilon=4$, we obtain an entire function whose range avoids $\overline{D(0,1)}$ and contains the complement of $D(0,5)$ such that the inverse image of $(z)$ never contains more than 16 elements, and so on. Of course, since $(\cos (\pi / n))^{n} \underset{n \rightarrow \infty}{\rightarrow} 1$, it follows from Theorem 8.13 that for every $\varepsilon>0$ there exists an entire function whose range avoids $\overline{D(0,1)}$ and contains the complement of $D(0,1+\varepsilon)$.

\section{REFERENCES}

1. N. H. Abel, Détermination d'une fonction au moyen d'une équation qui ne contient qu'une seule variable, Oeuvres complètes II, Christiania (1881), 36-39.

2. L. V. Ahlfors, The theory of meromorphic curves, Acta Soc. Sci. Fenn. Nova Ser. A 3 (1941), $1-31$.

3. M. Akkar, Sur la structure des algèbres topologiques localement multiplicativement convexes, C. R. Acad. Sci. Paris 279 (1974), 941-944.

4. H. Alexander, Explicit embedding of the (punctured) disc into $\mathbf{C}^{2}$, Notices Amer. Math. Soc. 24 (1977), A 472.

5. G. R. Allan, Embedding the algebra of all formal power series in a Banach algebra, Proc. London Math. Soc. (3) 25 (1972), 329-340.

6. N. U. Arakelian, Uniform approximation on closed sets by entire functions, Izv. Akad. Nauk SSSR 28 (1964), 1187-1206 (Russian).

7. N. U. Arakelian and P. Gauthier, On tangential approximation by entire functions, Izv. Akad. Nauk Armyan. SSSR (6) 17 (1982), 421-441; English transl. in Soviet J. Contemporary Math. 17 (1982), 1-22.

8. R. Arens, Dense inverse limit rings, Michigan Math. J. 5 (1958), 169-182.

9. V. I. Arnold, Chapitres supplémentaires a la théorie des équations différentielles, Editions de Moscou, 1978.

10. H. Bass, E. H. Connell and D. Wright, The jacobian conjecture. Reduction of degree and expansion of the inverse. Bull. Amer. Math. Soc. (N.S.) 7 (1982), 287-330.

11. L. Bieberbach, Beispiel zweier ganzer Funktionen zweier komplexer Variablen, welche eine schlichte volumtreue Abbildung des $R^{4}$ auf einen Teil seiner selbst vermitteln, S. B. Preuss Akad. Wiss. $14 / 15$ (1933), 476-479.

12. G. R. Belitskii, Normal forms, invariants, and local mappings, Naukova Dumka, Kiev, 1979 (Russian).

13. S. Bochner and W. Martin, Several complex variables, Princeton Univ. Press, Princeton, N. J., 1948.

14. E. Borel, Sur les zéros des fonctions entières, Acta Math. 20 (1897), 357-396.

15. N. Bourbaki, Espaces vectoriels topologiques, Chapitre II, Masson, Paris, 1981.

16. Topologie générale, Chapitre II, Hermann, Paris, 1960.

17. A. D. Bruno, Analytic forms of differential equations, Trudy Moskov. Mat. Obshch. 25 (1971), 120-162; English transl. in Trans. of the Moscow Math. Soc. 25 (1971), 131-288.

18. D. Burns, Curvatures of Monge-Ampere foliations and parabolic manifolds, Ann. of Math. (2) 115 (1982), 349-373.

19. C. Camacho, On the local structure of conformal mappings and holomorphic vector fields in $\mathbf{C}^{2}$, Astérisque 59-60 (1978), 83-94.

20. C. Camacho and P. Sad, Topological classification and bifurcation of holomorphic flows with resonance in $\mathrm{C}^{2}$, Invent. Math. 67 (1982), 447-472.

21. J. Carlson and P. Griffiths, $A$ defect relation for equidimensional holomorphic mappings between algebraic varieties, Ann. of Math. (2) 95 (1972), 557-584.

22. H. Cartan, Sur les systèmes de fonctions holomorphes à variétés lacunaires et leurs applications, Ann. Sci. École Norm. Sup. 45 (1928), 256-346. 
23. Sur les fonctions de deux variables complexes: les transformations d'un domaine borné $D$ en un domaine intérieur à D, Bull. Soc. Math. France 58 (1930), 199-219.

24. S. S. Chern, Differential geometry: its past and future, Actes du Congrès International des Mathématiciens (Nice, 1970), Gauthier-Villars, Paris, 1971, Tome 1, pp. 41-53.

25. B. Chevreau and J. Esterle, Normal forms for germs of invertible analytic mappings $\left(\mathbf{C}^{p}, 0\right) \rightarrow$ $\left(\mathrm{C}^{p}, 0\right)$ (in preparation)

26. D. Clayton, A reduction of the continuous homomorphism problem for F-algebras, Rocky Mountain J. Math. 5 (1975), 337-344.

27. J. B. Conway, Functions of one complex variable, 2nd ed., Graduate Texts in Math., no. 11, Springer-Verlag, Berlin and New York, 1978.

28. D. Couty, Sur l'image d'une fonction entière de deux variables complexes, Proc. Edinburgh Math. Soc., 27 (1984), 327-331.

29. H. G. Dales, A discontinuous homomorphism from $C(X)$, Amer. J. Math. 101 (1979), $647-734$.

30. B. Davis, Picard's theorem and Brownian motion, Trans. Amer. Math. Soc. 213 (1975), $353-362$.

31. B Brownian motion and analytic functions, Ann. Probab. 7 (1979), 913-932.

32. P. G. Dixon, Scalar homomorphisms on algebras of infinitely long polynomials with an application to automatic continuity theory, J. London Math. Soc. (2) 19 (1979), 488-496.

33. P. G. Dixon and D. H. Fremlin, A remark concerning multiplicative functionals on LMC algebras, J. London Math. Soc. 5 (1972), 231-232.

34. K. Diederich and N. Sibony, Strange complex structures on Euclidean spaces, J. Reine Angew. Math. 311 / 312 (1979), 397-407.

35. A. Douady, Systèmes dynamiques holomorphes, Séminaire Bourbaki no $599(1982 / 3)$, Astérisque, 105-106 (1983), 39-63.

36. J. Dufresnoy, Théorie nouvelle des familles complexes normales. Applications à l'étude des fonctions algébroides, Ann. Sci. École Norm. Sup. (3) 61 (1944), 1-44.

37. H. Dulac, Recherche sur les points singuliers des équations différentielles, J. École Polytechnique (2) 9 (1904), 1-125.

38. _ Sur les cycles limites, Bull. Soc. Math. France 51 (1923), 45-188.

39. N. Dunford and J. T. Schwartz, Linear operators. Part I: General theory, Pure and Appl. Math., vol. 7, Interscience, New York, 1958.

40. J. Ecalle, Théorie itérative, introduction à la théorie des invariants holomorphes, J. Math. Pures Appl. 54 (1975), 183-258.

41. P. M. Elizarov and Yu. S. Il'yashenko, Remarks on the orbital analytic classification of germs of vector fields, Mat. Sb. 121 (1983), 111-130 (Russian).

42. J. Esterle, Injection de semi-groups divisibles dans des algèbres de convolution et construction d'homomorphismes discontinus de $\mathscr{C}(K)$, Proc. London Math. Soc. (3) 36 (1978), 59-85.

43. Elements for a classification of commutative radical Banach algebras, Radical Banach Algebras and Automatic Continuity, Proceedings, Long Beach, 1981, Lecture Notes in Math., vol. 975, Springer-Verlag, Berlin and New York, pp. 1-65.

44 , Problème de Michael et fonctions entières de plusieurs variables complexes, Proc.

Conf. Analyse Complexe en Plusieurs Variables, Toulouse, 1983, Lecture Notes in Math., vol.

1094, Springer-Verlag, Berlin and New York, 1984, pp. 65-83.

45. Mittag-Leffler methods in the theory of Banach algebras and a new approach to Michael's problem, Proc. Yale Conf. in honour of C. E. Rickart (1983), Contemp. Math., vol. 32, Amer. Math. Soc., Providence, R. I., 1984, pp. 107-129.

46. P. Fatou, Sur certaines fonctions uniformes de deux variables, C. R. Acad. Sci. Paris 175 (1922), 1030-1033.

47. J. P. Francoise, Sur les formes normales des champs de vecteurs, Boll. Un. Mat. Ital. (5) 17 A (1980), 60-66.

48. W. H. Fuchs, Théorie de l'approximation des fonctions d'une variable complexe, Presses de l'Université de Montréal, Montréal, 1968.

49. P. Gauthier and Ngô Van Quê, Problème de surjectivité des applications holomorphes, Ann. Sci. Norm. Sup. Pisa (2) 27 (1973), 555-559.

50. M. Green, Holomorphic maps into complex projective spaces omitting hyperplanes, Trans. Amer. Math. Soc. 169 (1972), 89-103. 
51. P. Griffiths, Entire holomorphic mappings in one and several variables, Ann. of Math. Studies no. 85, Princeton Univ. Press, Princeton, N. J., 1976.

52. L. Gruman, Value distribution for holomorphic maps in $\mathbf{C}^{n}$, Math. Ann. 245 (1979), 199-218. 53. Ensembles exceptionnels pour les applications holomorphes dans $\mathbf{C}^{n}$, Séminaire

Lelong-Dolbeaut-Skoda, Années 1981/83, Lecture Notes in Math., vol. 1028, Springer-Verlag, Berlin and New York, pp. 125-162.

54. R. C. Gunning and H. Rossi, Analytic functions of several complex variables, Prentice-Hall, Englewood Cliffs, N. J., 1965.

55. S. Hitotomatu, Proposed problems and solutions, Sûgaku, Math. Soc. Japan 10 (1958), 195. (Japanese)

56. L. Hörmander, An introduction to complex analysis in several variables, North-Holland, Amsterdam, London, New York, 1973.

57. J. E. Humphreys, Linear algebraic groups, Graduate Texts in Math., no. 21, Springer-Verlag, Berlin and New York, 1975.

58. G. Julia, Mémoire sur l'itération des fractions rationnelles, Journal de Math. $8^{e}$ Sér. 1 (1918), $47-245$.

59. Sur quelques problèmes relatifs à itération des fractions rationnelles, C. R. Acad. Sci. Paris Ser. A 168 (1919), 147-148.

60. M. Kato, A generalization of Bieberbach's example, Proc. Japan Acad. 50 (1974), 329-333.

61. K. Kodaira, Holomorphic mappings of polydiscs into compact complex manifolds, J. Differential Geom. 6 (1971), 33-46.

62. M. Koenigs, Recherches sur les intégrales de certaines équations fonctionnelles, Ann. École Norm. Sup. 1 (1882), 3-41.

63. M. Kuczma, Functional equations, P. W. N., Warsaw, 1968.

64. H. B. Laufer, Embedding annuli in $\mathbf{C}^{2}$, J. Analyse Math. 26 (1973), 187-215.

65. S. Lattès, Sur les formes réduites des transformations ponctuelles à deux variables, C. R. Acad.

Sci. Paris Ser. A 152 (1911), 1566-1569.

66. Sur l'itération des substitutions rationnelles à deux variables, C. R. Acad. Sci. Paris Ser. A 166 (1918), 151-153.

67. L. Leau, Étude sur les équations fonctionnelles à une ou plusieurs variables, Ann. Fac. Sci.

Toulouse Math. 11 (1897), 1-110.

68. B. Malgrange, Travaux d'Ecalle et Martinet-Ramis sur les systèmes dynamiques, Séminaire N. Bourbaki, $n^{\circ} 582$ (1981/82), Astérisque $92 / 93$ (1982), 59-73.

69. R. Mane, P. Sad and D. Sullivan, On the dynamics of rational maps, Ann. Sci. École Norm. Sup. 16 (1983), 193-217.

70. J. Martinet, Normalisation des champs de vecteurs holomorphes (d'après A. D. Brjuno), Séminaire Bourbaki $n^{\circ} 564(1980 / 81)$, Lecture Notes in Math., vol. 901, Springer-Verlag, Berlin and New York, 1981, pp. 55-70.

71. J. Martinet and J. P. Ramis, Problèmes de modules pour les équations différentielles linéaires du premier ordre, Inst. Hautes Études Sci. Publ. Math. 55 (1982), 63-164.

72. Classification analytique des équations différentielles non linéaires résonnantes $d u$ premier ordre, Ann. Sci. École Norm. Sup. 16 (1983), 571-621.

73. E. A. Michael, Locally multiplicatively-convex topological algebras, Mem. Amer. Math. Soc. No. 11 (1952).

74. T. T. Moh, On the jacobian conjecture and the configuration of roots, J. Reine Angew. Math. 340 (1983), 140-212.

75. R. Nevanlinna, Le théorème de Picard-Borel et la théorie des fonctions méromorphes, Gauthiers-Villars, Paris, 1929.

76. Y. Nishimura, Automorphismes analytiques admettant des sousvariétés de points fixes dans une direction transversale, J. Math. Kyoto Univ. (2) 23 (1983), 289-299.

77. __ Applications holomorphes injectives de $\mathbf{C}^{2}$ dans lui-même qui exceptent une droite complexe, J. Math. Kyoto Univ. (4) 24 (1984), 755-761.

78. T. Nishino, Proposed problems and solutions, Sûgaku, Math. Soc. Japan 20 (1968), 125 (Japanese).

79. Le théorème de Borel et le théorème de Picard, C. R. Acad. Sci. Paris (14) 299 (1984), $667-668$. 
80. E. Picard, Sur une classe de transcendantes nouvelles, Acta Math. 18 (1894), 133-154.

81. __ Sur une classe de transcendantes nouvelles. II, Acta Math. 23 (1900), 333-337.

82. - Sur une classe de surfaces algébriques dont les coordonnées s'expriment par des fonctions de deux paramètres, Bull. Soc. Math. France 28 (1900), 17-25.

83. Sur certaines équations fonctionnelles et sur une classe de surfaces algébriques, C. R.

Acad. Sci. Paris 139 (1904), 5-9.

84. H. Poincaré, Sur les propriétés des fonctions définies par les équations aux différences partielles,

Thèse, Gauthier-Villars, Paris, 1879.

85. __ Sur une classe nouvelle de transcendantes uniformes, J. de Math. 6 (1890), 313-365.

86. M. Rajoelina, Eléments de Cohen et fonctions extérieures de l'algébre du disque, J. Functional Analysis (to appear).

87. L. Reich, Das typenproblem beiformal biholomorphen Abbildungen mit anziehendem Fixpunt, Math. Ann. 179 (1969), 227-250.

88. , Normalformen biholomorphen Abbildungen mit anziehendem Fixpunt, Math. Ann. 180 (1969), 233-255.

89. L. I. Ronkin, Introduction to the theory of entire functions in several complex variables, Transl. Math. Monographs, vol. 44, Amer. Math. Soc., Providence, R.I., 1974.

90. A. Roth, Approximationseigenschaften und Strahlen grenzwerte meromorpher und ganzer Funktionen, Comment Math. Helv. 11 (1938), 77-125.

91. W. Rudin, Private communication.

92. H. Russmann, On the convergence of power series transformations of analytic mappings near a fixed point into a normal form (preprint) Inst. Hautes Études Sci. Publ. Math. I.H.E.S. 1977.

93. A. Sadullaev, On Fatou's example, Mat. Zametki 6 (1969), 437-441 (Russian), (translated in Math. Notes 6 (1969), 717-719).

94. M. Schottenloher, Michael's problem and algebras of holomorphic functions, Arch. Mat. 37 (1981), 241-247.

95. E. Schröder, Über unendlich viele Algorithmen zur Auflosung der Gleichungen, Math. Ann. 2 (1870), 317-365.

96. , Über iterierte Funktionen, Math. Ann. 3 (1871), 296-322.

97. A. A. Sherbakov, Germs of maps, analytically not equivalent with their formal normal forms,

Funktsional Anal. i Prilozhen. (2) 16 (1982), 94-95 (Russian).

98. N. Sibony and Pit-Mann Wong, Some remarks on the Casorati-Weierstrass theorem, Ann.

Polon. Math. 39 (1981), 165-174.

99. C. L. Siegel, Iteration of analytic functions, Ann. of Math. (2) 43 (1942), 607-612.

100. H. Skoda, Fibrés holomorphes à base et à fibre de Stein, C. R. Acad. Sci. Paris 284 (1977), 1199-1202.

101. J.-L. Stehlé, Plongements du disque dans $\mathbf{C}^{2}$, Séminaire P. Lelong (Analyse) Lecture Notes in Math., vol. 275, Springer-Verlag, Berlin and New York, 1970, pp. 119-130.

102. S. Sternberg, Local contractions and a theorem of Poincaré, Amer. J. Math. 79 (1957), 809-823.

103. Infinite Lie groups and the formal aspects of dynamical systems, J. Math. Mech. (3) 10 (1961), 451-474.

104. W. Stoll, The characterization of strictly parabolic manifolds, Ann. Sci. Norm. Sup. Pisa Sér.

A (1) 7 (1980), 87-154.

105. D. Sullivan and W. Thurston, Extending holomorphic motions (to appear).

106. A. Vitter, The lemma of the logarithmic derivative in several complex variables, Duke Math. J. 44 (1977), 89-104.

107. S. M. Voronin, Analytic classification of germs of conformal mappings $(\mathbf{C}, 0) \rightarrow(\mathbf{C}, 0)$ with identity linear part, Funktsional. Anal. i Prilozhen. 15 (1981), no. 1, 1-17; English transl. in Functional Anal. Appl. 15 (1981), 1-13.

108. S. Wang, A jacobian criterion for separability, J. Algebra 65 (1980), 453-494.

109. H. Wu, An n-dimensional extension of Picard's theorem, Bull. Amer. Math. Soc. 75 (1969), 1357-1361.

110. The equidistribution theory of holomorphic curves, Ann. of Math. Studies, No. 64, Princeton Univ. Press, Princeton, N. J., 1970.

111. Mapping of Riemann surfaces (Nevanlinna theory), Proc. Sympos. Pure Math., vol. 11, Amer. Math. Soc., Providence, R. I., 1968, pp. 480-532. 
112. M. G. Zaidenberg, Picard's theorem and hyperbolicity, Siberian Math. J. (6) 24 (1983), 44-55 (Russian).

113. E. Zehnder, A simple proof of a generalization of a theorem by C. L. Siegel, Geometry and Topology III (M. de Carmo and J. Palis, Jr., eds.), Lecture Notes in Math., vol. 597, Springer-Verlag, Berlin and New York, 1977, pp. 855-866.

114. F. Zouakia, Semi-groupes réels dans les algèbres de Banach commutatives (to appear).

Department of Pure Mathematics, University of Sheffield, Sheffield, S3 7RH, EngLAND

Laboratoire associle aU CNRS $\mathrm{N}^{\circ}$ 226, U.E.R. DE Mathematiques et Informatique, Universite de Bordeaux I, 351, cours de La Liberation, 33405 TAlence, France 
